\title{
Insights into the Hydrogen-Abstraction Reactions of Diol Dehydratase: Relevance to the Catalytic Mechanism and Suicide Inactivation
}

\author{
Gregory M. Sandala, ${ }^{\dagger, \diamond}$ David M. Smith", , Michelle L. Coote, ${ }^{\triangleright, \uparrow}$ Bernard T. Golding, ${ }^{\S}$ \\ and Leo Radom ${ }^{\dagger, \lambda, \pi, *}$ \\ School of Chemistry, University of Sydney, Sydney, NSW 2006, Australia, Research School of \\ Chemistry, Australian National University, Canberra, ACT 0200, Australia, Rudjer Boskovic \\ Institute, 10002 Zagreb, Croatia, School of Natural Sciences-Chemistry, University of Newcastle \\ upon Tyne, Bedson Building, Newcastle upon Tyne, NE1 7RU, United Kingdom, and ARC Centre \\ of Excellence in Free Radical Chemistry and Biotechnology
}

\section{SUPPORTING INFORMATION}

(Tables S1-S2 and complete citations for references 33 and 34,

Total 36 pages including this page)

\footnotetext{
${ }^{\dagger}$ University of Sydney

$\checkmark$ Australian National University

- Rudjer Boskovic Institute

${ }^{\S}$ University of Newcastle upon Tyne

'ARC Centre of Excellence in Free Radical Chemistry and Biotechnology

*E-mail: david.smith@irb.hr; radom@chem.usyd.edu.au
} 


\section{TABLE S1: GAUSSIAN Archive Entries for the B3-LYP/6-31G(d,p) Optimized Geometries}

$1 \mathbf{a}$

$1 \backslash 1 \backslash G I N C-L C 103 \backslash F r e q \backslash R B 3 L Y P \backslash 6-31 G(d, p) \backslash C 2 H 602 \backslash G M S 501 \backslash 16-O c t-2003 \backslash 0 \backslash \backslash \# N$ GEOM=ALLCHECK GUESS=READ SCRF=CHECK TEST GENCHK RB3LYP/6-31G (D,P)FREQ $\backslash \backslash 1,2$-dihydroxyethane1 b3lyp/6-31G(d,p) opt freq $\backslash \backslash 0,1 \backslash \mathrm{C}, 0.6846871585,0$ $.6099678035,-0.2627758529 \backslash \mathrm{C},-0.7404008459,0.5721429676,0.2655051471 \backslash 0$, $1.318992022,-0.5753752696,0.2223301471 \backslash 0,-1.4224559788,-0.5822059538,-$ $0.1895908529 \backslash \mathrm{H},-0.7138378405,0.6188139646,1.3664291471 \backslash \mathrm{H},-1.2952317453$ $, 1.4448610315,-0.0944678529 \backslash \mathrm{H}, 0.6635081602,0.6247938059,-1.3619138529 \backslash$ $\mathrm{H}, 1.200790263,1.516912744,0.0886721471 \backslash \mathrm{H},-0.8242870638,-1.3199150227,0$ $.0013401471 \backslash \mathrm{H}, 2.1310520056,-0.7174813631,-0.2783498529 \backslash \backslash$ Version=x86-Li nux-G03RevB.03 \State $=1-\mathrm{A} \backslash \mathrm{HF}=-230.2573838 \backslash \mathrm{RMSD}=7.254 \mathrm{e}-10 \backslash \mathrm{RMSF}=4.175 \mathrm{e}-06$ $\backslash \mathrm{Dipole}=0.918257,0.2940294,0.2296127 \backslash \mathrm{PG}=\mathrm{C} 01[\mathrm{X}(\mathrm{C} 2 \mathrm{H} 6 \mathrm{O} 2)] \backslash \backslash @$

\section{TS: $1 a \rightarrow 3 a$}

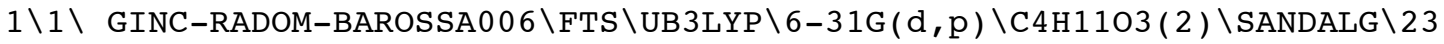

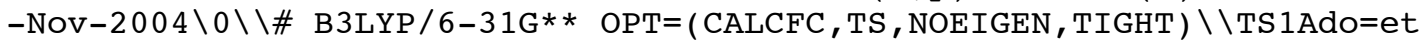

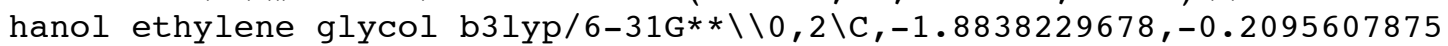
$,-0.0886086444 \backslash 0,-3.1720483792,0.1858474146,-0.5452299871 \backslash \mathrm{H},-3.3349163$ $167,1.0528881575,-0.1472608907 \backslash \mathrm{H},-1.6312959462,-1.1361922289,-0.613302$ $0768 \backslash \mathrm{H},-1.8896773157,-0.4220108532,0.9921165458 \backslash \mathrm{C},-0.8392654111,0.8418$ $457106,-0.37736264 \backslash 0,-1.1675547206,2.0032654739,0.343441458 \backslash \mathrm{H},-0.64908$ $93834,2.742378318,0.0022316781 \backslash \mathrm{H}, 0.3143162239,0.3888821406,0.037153731$ $\backslash \mathrm{H},-0.7102903874,1.0279071198,-1.4525816421 \backslash \mathrm{C}, 2.04735213,-1.2192130306$ $,-0.181225183 \backslash \mathrm{C}, 1.5791365935,-0.0066705136,0.574237982 \backslash \mathrm{H}, 2.1151744128$, $-0.9887134,-1.2572071336 \backslash 0,3.3255378223,-1.610399772,0.33249229 \backslash \mathrm{H}, 1.35$ $30067118,-0.1831956796,1.6267566925 \backslash \mathrm{H}, 2.1975373415,0.8811297715,0.4292$ $936945 \backslash \mathrm{H}, 1.3163447881,-2.0365399905,-0.0691199272 \backslash \mathrm{H}, 3.6110100232,-2.39$ $464656,-0.1559598469 \backslash \backslash$ Version=x86-Linux-G03RevB. 02 $\backslash$ State $=2-A \backslash H F=-384.6$ $113666 \backslash \mathrm{S} 2=0.75679 \backslash \mathrm{S} 2-1=0 . \backslash \mathrm{S} 2 \mathrm{~A}=0.750023 \backslash \mathrm{RMSD}=3.897 \mathrm{e}-09 \backslash \mathrm{RMSF}=1.721 \mathrm{e}-06 \backslash \mathrm{D}$ ipole $=0.3336865,0.309941,-0.4968816 \backslash \mathrm{PG}=\mathrm{C} 01[\mathrm{X}(\mathrm{C} 4 \mathrm{H} 1103)] \backslash \backslash @$

\section{3a}

$1 \backslash 1 \backslash G I N C-L C 85 \backslash F O p t \backslash U B 3 L Y P \backslash 6-31 G(d, p) \backslash C 2 H 5 O 2(2) \backslash G M S 501 \backslash 16-0 c t-2003 \backslash 0 \backslash \backslash \#$ B3LYP/6-31G(D,P) OPT=(TIGHT,MAXCYCLE=100) FREQ TEST MAXDISK=26214400\\ dihydroxyethane radical b3lyp/6-31G(d,p) opt freq $\backslash \backslash 0,2 \backslash \mathrm{C}, 0.7373400859$, $0.5821731587,-0.2622973548 \backslash \mathrm{C},-0.6816019268,0.6501536639,0.1914114348 \backslash 0$ $, 1.4365648724,-0.5320624608,0.104076246 \backslash \mathrm{H}, 0.7650894517,-1.2173656526,0$ $.269172713 \backslash \mathrm{H}, 1.3537897125,1.4679403731,-0.3754200319 \backslash 0,-1.3271357747$, $0.6152256011,-0.0362061045 \backslash \mathrm{H},-1.3494597082,-0.7472160299,-0.9956254499$ $\backslash \mathrm{H},-0.7708081059,0.7985794021,1.2801027536 \backslash \mathrm{H},-1.2084730863,1.482405466$ $8,-0.2958755966 \backslash \backslash$ Version=x86-Linux-G03RevB.03 \State $=2-A \backslash H F=-229.600149$ $\backslash \mathrm{S} 2=0.753065 \backslash \mathrm{S} 2-1=0 . \backslash \mathrm{S} 2 \mathrm{~A}=0.750007 \backslash \mathrm{RMSD}=2.837 \mathrm{e}-09 \backslash \mathrm{RMSF}=1.947 \mathrm{e}-06 \backslash \mathrm{Dipole}$ $=-0.3597031,0.2537363,-0.3119129 \backslash \mathrm{PG}=\mathrm{C} 01[\mathrm{X}(\mathrm{C} 2 \mathrm{H} 5 \mathrm{O} 2)] \backslash \backslash \mathrm{a}$ 


\section{4a}

$1 \backslash 1 \backslash G I N C-L C 79 \backslash F O p t \backslash U B 3 L Y P \backslash 6-31 G(d, p) \backslash C 2 H 5 O 2$ ( 2$) \backslash G M S 501 \backslash 29-N o v-2003 \backslash 0 \backslash \backslash \#$ P B3LYP/6-31G(D,P) OPT=(TIGHT) FREQ TEST MAXDISK=26214400 GFINPUTIOP(

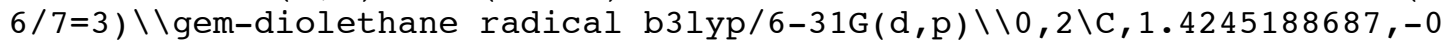
$.0905794869,-0.0536664395 \backslash \mathrm{H}, 2.1237786375,0.6804688173,0.2461599298 \backslash \mathrm{H}, 1$ $.7586361548,-0.9250596891,-0.6580724643 \backslash \mathrm{C},-0.0016407348,0.0152504032,0$ $.3563399519 \backslash 0,-0.6617502136,-1.1461389327,-0.0969044933 \backslash \mathrm{H},-0.098114843$ $6,0.126696031,1.44849059 \backslash 0,-0.621074386,1.1980195042,-0.141147367 \backslash \mathrm{H},-0$ $.4669795405,1.2045967309,-1.0981800801 \backslash \mathrm{H},-1.5919924148,-1.0497719591,0$ $.1499758327 \backslash \backslash$ Version=x86-Linux-G03RevB .03 $\backslash$ State=2-A \HF=-229.5992417\S2 $=0.753791 \backslash \mathrm{S} 2-1=0 . \backslash \mathrm{S} 2 \mathrm{~A}=0.750009 \backslash \mathrm{RMSD}=2.579 e-09 \backslash \mathrm{RMSF}=5.776 e-07 \backslash \mathrm{Dipole}=0$. $0088366,0.0284326,-0.1055384 \backslash \mathrm{PG}=\mathrm{C} 01[\mathrm{X}(\mathrm{C} 2 \mathrm{H} 5 \mathrm{O} 2)] \backslash \backslash @$

\section{TS: $4 a \rightarrow 5 a$}

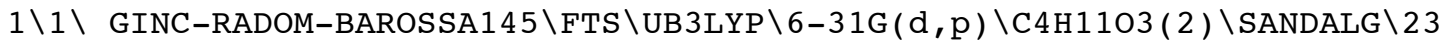

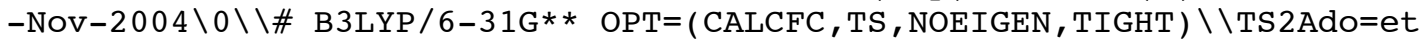

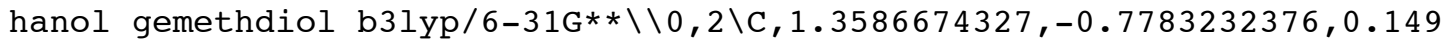
$5512748 \backslash \mathrm{H}, 1.9510736506,-1.2993872211,0.901778973 \backslash \mathrm{H}, 0.0779982711,-0.929$ $9656931,0.5517808146 \backslash \mathrm{C}, 1.6493207774,0.6971273974,0.076605001 \backslash 0,0.77772$ $40623,1.2488602224,-0.8928006168 \backslash \mathrm{H}, 1.5046166976,1.1749264934,1.0554966$ $696 \backslash 0,3.003530471,0.977949578,-0.2387824433 \backslash \mathrm{H}, 3.1946183372,0.520303931$ $9,-1.0714788847 \backslash \mathrm{H}, 0.9806747197,2.1935329164,-0.9441398289 \backslash \mathrm{H}, 1.38914738$ $37,-1.2697697606,-0.8263063569 \backslash \mathrm{C},-2.0702953043,-0.5190635675,-0.155955$ $8306 \backslash \mathrm{C},-1.2058324384,-1.0581663559,0.9541934667 \backslash \mathrm{H},-1.9038428683,-1.103$ $6053698,-1.0749153313 \backslash 0,-3.4369065679,-0.6057961972,0.2633012753 \backslash \mathrm{H},-1$. $2539688044,-0.4764514946,1.8769228434 \backslash \mathrm{H},-1.3227542018,-2.1272485412,1$. $1418237039 \backslash \mathrm{H},-1.7828333491,0.5186201662,-0.3754338488 \backslash \mathrm{H},-3.9806763642$, $-0.218509672,-0.4356379469 \backslash \backslash$ Version=x86-Linux-G03RevB. 02 $\backslash$ State $=2-A \backslash H F=$ $-384.6246412 \backslash \mathrm{S} 2=0.756971 \backslash \mathrm{S} 2-1=0 . \backslash \mathrm{S} 2 \mathrm{~A}=0.750026 \backslash \mathrm{RMSD}=7.255 \mathrm{e}-09 \backslash \mathrm{RMSF}=1.29$ $3 e-06 \backslash \mathrm{Dipole}=0.2549031,0.2631202,-0.6255309 \backslash \mathrm{PG}=\mathrm{C} 01[\mathrm{X}(\mathrm{C} 4 \mathrm{H} 1103)] \backslash \backslash @$

\section{$5 a$}

$1 \backslash 1 \backslash \mathrm{GINC}-\mathrm{SC} 3 \backslash \mathrm{FOpt} \backslash \mathrm{RB} 3 \mathrm{LYP} \backslash 6-31 \mathrm{G}(\mathrm{d}, \mathrm{p}) \backslash \mathrm{C} 2 \mathrm{H} 6 \mathrm{O} 2 \backslash \mathrm{GMS} 501 \backslash 08-\mathrm{Dec}-2003 \backslash 0 \backslash \backslash \# \mathrm{~PB} 3$ LYP /6-31G(D,P) OPT=(TIGHT,MAXCYC=100) FREQ TEST MAXDISK=31457280 GFINP

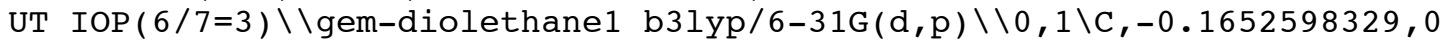
$.294509144,-1.3676259284 \backslash \mathrm{C},-0.1661370075,0.2882474345,0.1520703085 \backslash 0,1$ $.1846270363,0.2795409943,0.566437715 \backslash \mathrm{H}, 1.1785559244,0.2645869318,1.533$ $7899103 \backslash \mathrm{O},-0.8791184962,-0.8041938664,0.6957908791 \backslash \mathrm{H},-0.4600331721,-1$. $6078802166,0.3532847372 \backslash \mathrm{H},-0.6931490663,1.1699194419,0.5438028245 \backslash \mathrm{H}, 0$. $34805676,1.1805092142,-1.7500764158 \backslash \mathrm{H}, 0.3619318908,-0.5902603794,-1.74$ $1936682 \backslash \mathrm{H},-1.1910496147,0.2838085143,-1.7433594077 \backslash \backslash$ Version=DEC $-\mathrm{AXP}-\mathrm{OS}$ $\mathrm{F} / 1-\mathrm{G} 03 \mathrm{RevB} .03 \backslash \mathrm{State}=1-\mathrm{A} \backslash \mathrm{HF}=-230.2755365 \backslash \mathrm{RMSD}=1.919 \mathrm{e}-09 \backslash \mathrm{RMSF}=1.798 \mathrm{e}-06$ $\backslash$ Dipole $=0.0437383,-0.0879945,-0.0173994 \backslash \mathrm{PG}=\mathrm{C} 01$ [X(C2H6O2) ] \\@

\section{2a}

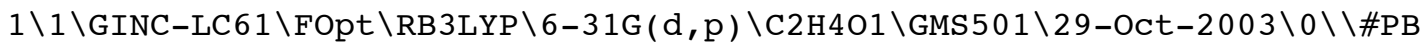
3LYP/6-31G(D,P) OPT=(TIGHT, READFC) FREQ TEST MAXDISK=19660800 GUESS=RE

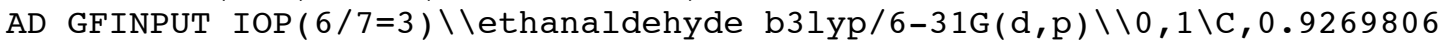
$606,-0.7294793263,0 . \backslash \mathrm{C}, 0.0053674452,0.4631479279,0 . \backslash 0,-1.2036047885,0$. $398565881,0 . \backslash \mathrm{H}, 0.5204029884,1.4513440402,0 . \backslash \mathrm{H}, 1.5812781437,-0.69252306$ $97,0.8796768448 \backslash \mathrm{H}, 1.5812781437,-0.6925230697,-0.8796768448 \backslash \mathrm{H}, 0.3517903$ $969,-1.656836559,0 . \backslash \backslash$ Version=x86-Linux-G03RevB . 03 \State=1-A $\backslash$ HF $=-153.8$ $357277 \backslash \mathrm{RMSD}=7.876 \mathrm{e}-09 \backslash \mathrm{RMSF}=2.215 \mathrm{e}-06 \backslash \mathrm{Dipole}=1.0332679,-0.1360127,0 . \backslash \mathrm{PG}$ $=\mathrm{CS} \quad[\mathrm{SG}(\mathrm{C} 2 \mathrm{H} 2 \mathrm{O} 1), \mathrm{X}(\mathrm{H} 2)] \backslash \backslash @$ 


\section{$1 \mathrm{~b}$}

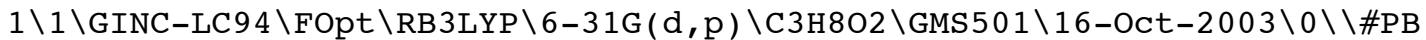
3LYP $/ 6-31 \mathrm{G}(\mathrm{D}, \mathrm{P})$ OPT=(TIGHT, MAXCYCLE=100) FREQ TEST MAXDISK=30146560GF INPUT IOP(6/7=3) \\oxygens gauche, methyl anti a2 conformer-H inpage $31 \mathrm{yp} / 6-31 \mathrm{G}(\mathrm{d}, \mathrm{p}) \backslash \backslash 0,1 \backslash \mathrm{C},-0.4684074294,0.0521124594,-0.3416584183 \backslash \mathrm{C}, 0.71$ $60611187,-0.7439030349,0.1995763755 \backslash 0,1.8912871716,-0.0147960467,-0.16$ $23885881 \backslash \mathrm{H}, 0.6222563148,-0.8263171213,1.292504072 \backslash \mathrm{H}, 0.7271380614,-1.75$ $799394,-0.2278767287 \backslash \mathrm{C},-1.8064669271,-0.5650266888,0.0377231948 \backslash 0,-0.4$ $29209804,1.3732194443,0.1814532389 \backslash \mathrm{H},-0.375127934,0.0764294356,-1.4414$ $10518 \backslash \mathrm{H},-1.9083969962,-0.6196245499,1.1263669832 \backslash \mathrm{H},-2.6225955928,0.051$ $690907,-0.3473550156 \backslash \mathrm{H},-1.9084363563,-1.5725058188,-0.3783598976 \backslash \mathrm{H}, 2.6$ $295736692,-0.3365810177,0.3682838788 \backslash \mathrm{H}, 0.4918493199,1.6584185111,0.081$ $4831075 \backslash \backslash$ Version=x86-Linux-G03RevB.03 SState $=1-\mathrm{A} \backslash \mathrm{HF}=-269.5800463 \backslash \mathrm{RMSD}=5$ $.355 e-09 \backslash \mathrm{RMSF}=2.718 \mathrm{e}-06 \backslash \mathrm{Dipole}=0.5632987,-0.7455832,0.2383958 \backslash \mathrm{PG}=\mathrm{C} 01[$ $\mathrm{X}(\mathrm{C} 3 \mathrm{H} 8 \mathrm{O} 2)] \backslash \backslash @$

\section{TS: $1 b \rightarrow 3 b$}

$1 \backslash 1 \backslash G I N C-B A R O S S A 023 \backslash F T S \backslash U B 3 L Y P \backslash 6-31 G(d, p) \backslash C 5 H 1303(2) \backslash S A N D A L G \backslash 20-A p r-20$ $05 \backslash 0 \backslash \backslash \#$ B3LYP/6-31G* * OPT=(TS, NOEIGEN, CALCFC) FREQ=NORAMAN \TS2diolet


$.1552435493 \backslash \mathrm{C},-0.9026162751,-0.4952490279,0.4979263642 \backslash 0,-1.196461882$, $-0.8256548951,1.8330397879 \backslash \mathrm{H},-1.1402426664,-1.3056547373,-0.2062490233$ $\backslash \mathrm{H}, 0.3872785742,-0.2991373771,0.3842595835 \backslash \mathrm{C},-1.3838472564,1.22998568$, $-1.2845581258 \backslash \mathrm{O},-3.0259967751,0.6319173994,0.3306619726 \backslash \mathrm{H},-1.244253546$ $1,1.5775329392,0.8398215166 \backslash \mathrm{H},-1.7182512301,0.4513725943,-1.9776779325$ $\backslash \mathrm{H},-1.9552359697,2.1375428374,-1.4949572167 \backslash \mathrm{H},-0.3248587408,1.43596740$ $33,-1.4660928925 \backslash \mathrm{H},-0.951477468,-1.7455112767,1.9915989624 \backslash \mathrm{H},-3.136813$ $3159,0.2383694548,1.2084494013 \backslash \mathrm{C}, 2.3536218151,-0.5981647417,-0.9091517$ $154 \backslash \mathrm{C}, 1.8012159677,-0.1154801226,0.4032634337 \backslash \mathrm{H}, 1.9207112221,-0.013634$ $4695,-1.7373114442 \backslash 0,3.7784970278,-0.459000784,-0.883319781 \backslash \mathrm{H}, 2.103386$ $7258,-0.7099508403,1.2673007227 \backslash \mathrm{H}, 1.9203599236,0.9543727835,0.58116413$ $25 \backslash \mathrm{H}, 2.0690706016,-1.650869102,-1.0727146579 \backslash \mathrm{H}, 4.1188074714,-0.7599753$ $839,-1.7369880248 \backslash \backslash$ Version=IA32L-G03RevC . 02 \State=2-A \HF=-423.9335861 $\mathrm{S} 2=0.756801 \backslash \mathrm{S} 2-1=0 . \backslash \mathrm{S} 2 \mathrm{~A}=0.750023 \backslash \mathrm{RMSD}=7.423 e-09 \backslash \mathrm{RMSF}=3.666 \mathrm{e}-06 \backslash \mathrm{Dipole}=$ $0.1516431,-0.8519774,-0.2778073 \backslash \mathrm{PG}=\mathrm{C} 01 \quad[\mathrm{X}(\mathrm{C} 5 \mathrm{H} 13 \mathrm{O} 3)] \backslash \backslash @$

\section{3b}

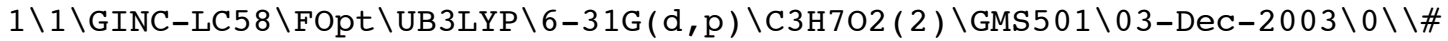
P B3LYP/6-31G(D,P) OPT=(TIGHT, MAXCYC=100) FREQ TEST MAXDISK=26214400G FINPUT IOP(6/7=3) \dihydroxypropane radical C1 symmetry b3lyp/6-31G(d, p) Hre removed $\mathrm{R}$-conformer $\backslash \backslash 0,2 \backslash \mathrm{C},-0.4316642743,0.0320280073,-0.326890$ $4794 \backslash \mathrm{C}, 0.7754359724,-0.6386619334,0.2349896668 \backslash 0,1.9594282461,-0.07723$ $07847,-0.1912689675 \backslash \mathrm{C},-1.7130124734,-0.7250011352,-0.0053190166 \backslash 0,-0.5$ $929001634,1.3637506612,0.2096657882 \backslash \mathrm{H},-0.3063186251,0.0984097317,-1.42$ $02029442 \backslash \mathrm{H},-1.8365729033,-0.8207343694,1.0782330744 \backslash \mathrm{H},-2.5749943187,-0$ $.1791930341,-0.3968285756 \backslash \mathrm{H},-1.6950997652,-1.7248915894,-0.4478055848 \backslash$ $\mathrm{H}, 2.6913255014,-0.455422913,0.3118427766 \backslash \mathrm{H}, 0.247694876,1.8185613553,0$. $06129061 \backslash \mathrm{H}, 0.7571852255,-1.0390778259,1.2496150533 \backslash \backslash$ Version=x86-LinuxG03RevB.03 \State $=2-A \backslash H F=-268.917507 \backslash S 2=0.753215 \backslash \mathrm{S} 2-1=0 . \backslash \mathrm{S} 2 \mathrm{~A}=0.750007 \backslash \mathrm{R}$ $\mathrm{MSD}=7.284 \mathrm{e}-09 \backslash \mathrm{RMSF}=1.585 e-06 \backslash \mathrm{Dipole}=0.7641683,-0.4498462,0.2350218 \backslash \mathrm{PG}=$ $\mathrm{C} 01[\mathrm{X}(\mathrm{C} 3 \mathrm{H} 7 \mathrm{O} 2)] \backslash \backslash @$ 


\section{4b}

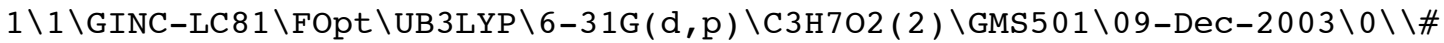
P B3LYP $/ 6-31 \mathrm{G}(\mathrm{D}, \mathrm{P})$ OPT $=(\mathrm{TIGHT}, \mathrm{MAXCYCLE}=100)$ FREQ MAXDISK $=32768000 \mathrm{GFIN}$


$40244311,-0.6278868961,-0.2116858595 \backslash C,-0.5136555185,-0.0204602291,0.3$ $262029217 \backslash 0,-0.682202813,1.3351408097,-0.0746902567 \backslash 0,-1.5913402826,-0$ $.8264233639,-0.0987565929 \backslash \mathrm{H},-0.4878454782,0.0523583565,1.428186691 \backslash \mathrm{C}, 2$ $.0657816228,-0.001484966,0.0497094752 \backslash \mathrm{H}, 2.7827022277,-0.2224471812,-0$. $7485901565 \backslash \mathrm{H}, 1.9727357107,1.0849754702,0.1453169515 \backslash \mathrm{H}, 2.5188139164,-0$. $3709323573,0.9859399869 \backslash \mathrm{H},-0.628543238,1.3374459632,-1.0428519673 \backslash \mathrm{H},-2$ $.3958661724,-0.4193191602,0.2509977947 \backslash \mathrm{H}, 0.6721253063,-1.6328281096,-0$ $.616783728 \backslash \backslash$ Version $=x 86-L$ inux $-G 03 R e v B .03 \backslash$ State $=2-A \backslash H F=-268.9235221 \backslash \mathrm{S} 2=$ $0.753781 \backslash \mathrm{S} 2-1=0 . \backslash \mathrm{S} 2 \mathrm{~A}=0.75001 \backslash \mathrm{RMSD}=6.435 \mathrm{e}-09 \backslash \mathrm{RMSF}=3.890 \mathrm{e}-06 \backslash \mathrm{Dipole}=0.10$ $85641,0.0160703,-0.0398751 \backslash \mathrm{PG}=\mathrm{C} 01[\mathrm{X}(\mathrm{C} 3 \mathrm{H} 7 \mathrm{O} 2)] \backslash \backslash @$

\section{TS: $4 b \rightarrow 5 b$}

$1 \backslash 1 \backslash G I N C-B A R O S S A 011 \backslash F T S \backslash U B 3 L Y P \backslash 6-31 G(d, p) \backslash C 5 H 1303(2) \backslash S A N D A L G \backslash 20-A p r-20$ $05 \backslash 0 \backslash \backslash \#$ B3LYP $/ 6-31 \mathrm{G} * *$ OPT=(TS, NOEIGEN, CALCFC) FREQ=NORAMAN $\backslash T S 2$ diolet

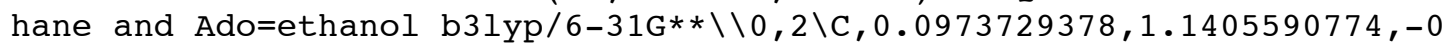
$.5254918917 \backslash \mathrm{C}, 1.3570918181,0.8732156486,0.2665446978 \backslash 0,2.1552543444,2$. $0329123245,0.4505091079 \backslash 0,0.9658216864,0.329411908,1.512086897 \backslash \mathrm{H}, 2.019$ $2309576,0.184937504,-0.2786598621 \backslash C, 0.3093761232,1.6808482455,-1.92435$ $56536 \backslash \mathrm{H},-0.6442623059,1.8226150259,-2.4414616519 \backslash \mathrm{H}, 0.8307881164,2.6456$ $110603,-1.9030207124 \backslash \mathrm{H}, 0.9194446206,0.9975613239,-2.5263167353 \backslash \mathrm{H}, 1.596$ $2504548,2.6826147244,0.9035289966 \backslash \mathrm{H}, 1.777286244,0.1662041792,2.0130117$ $189 \backslash \mathrm{H},-0.5081121254,-0.0348271892,-0.6476834787 \backslash \mathrm{H},-0.6195310644,1.7170$ $563748,0.0706075105 \backslash \mathrm{C},-1.5445882086,-1.7385505797,0.5439631791 \backslash \mathrm{C},-1.12$ $37419556,-1.2612487097,-0.8200214578 \backslash \mathrm{H},-2.2454132399,-1.0162138245,0.9$ $922903907 \backslash 0,-2.1630278778,-3.0233920605,0.397596425 \backslash \mathrm{H},-0.3535460813,-1$ $.8747441129,-1.2915283705 \backslash \mathrm{H},-1.9482765122,-1.0450534523,-1.501888063 \backslash \mathrm{H}$ $,-0.667065784,-1.789895174,1.2032517583 \backslash \mathrm{H},-2.3942427924,-3.3362659085$, $1.282495817 \backslash \backslash$ Version=IA32L-G03RevC.02 \State $=2-A \backslash H F=-423.9442509 \backslash \mathrm{S} 2=0.7$ $56977 \backslash \mathrm{S} 2-1=0 . \backslash \mathrm{S} 2 \mathrm{~A}=0.750026 \backslash \mathrm{RMSD}=8.151 \mathrm{e}-09 \backslash \mathrm{RMSF}=1.973 \mathrm{e}-06 \backslash \mathrm{Dipole}=0.1483$ $379,0.4429818,0.5878286 \backslash P G=C 01 \quad[X(C 5 H 1303)] \backslash \backslash @$

\section{$5 b$}

$1 \backslash 1 \backslash G I N C-S C 5 \backslash F O p t \backslash R B 3 L Y P \backslash 6-31 G(d, p) \backslash C 3 H 802 \backslash G M S 501 \backslash 03-D e c-2003 \backslash 0 \backslash \backslash$ \#PB3 LYP $/ 6-31 \mathrm{G}(\mathrm{D}, \mathrm{P}) \quad \mathrm{OPT}=(\mathrm{TIGHT}, \mathrm{READFC}, \mathrm{MAXCYCLE}=100) \quad$ FREQ MAXDISK $=32768000 \mathrm{G}$ UESS=READ GFINPUT IOP $(6 / 7=3) \backslash \backslash$ gem-diolpropane b3lyp/6-31G $(d, p) \backslash \backslash 0,1 \backslash \mathrm{C}$, $0.7278919831,-0.6699529508,-0.2620484964 \backslash \mathrm{C},-0.5116055735,-0.0059996742$ $, 0.3297145407 \backslash 0,-0.6663850399,1.3405752285,-0.0749621709 \backslash 0,-1.62232249$ $85,-0.7872692771,-0.0572589441 \backslash \mathrm{H},-0.4302002628,0.0546519314,1.42520896$ $37 \backslash \mathrm{C}, 2.0258941839,0.0514425331,0.1041021579 \backslash \mathrm{H}, 2.8878697833,-0.42696553$ $68,-0.3701087429 \backslash \mathrm{H}, 1.9931955439,1.0971007143,-0.2141074214 \backslash \mathrm{H}, 2.1936364$ $292,0.0410229835,1.1868734474 \backslash \mathrm{H},-0.7293336438,1.3325920915,-1.04215339$ $05 \backslash \mathrm{H},-2.4070256972,-0.3609206624,0.3147107094 \backslash \mathrm{H}, 0.7511138297,-1.710711$ $3194,0.0792333817 \backslash \mathrm{H}, 0.5973207639,-0.7061572617,-1.3524972407 \backslash \backslash$ Version= DEC-AXP-OSF $/ 1-G 03 R e v B .03 \backslash$ State $=1-A \backslash H F=-269.5924038 \backslash R M S D=3.457 e-09 \backslash$ RMSF $=2.546 e-06 \backslash \mathrm{Dipole}=-0.0323951,0.0199158,-0.0976567 \backslash \mathrm{PG}=\mathrm{C} 01[\mathrm{X}(\mathrm{C} 3 \mathrm{H} 8 \mathrm{O} 2)] \backslash \backslash$ a 


\section{2b}

$1 \backslash 1 \backslash G I N C-L C 70 \backslash F O p t \backslash R B 3 L Y P \backslash 6-31 G(d, p) \backslash C 3 H 601 \backslash G M S 501 \backslash 14-N o v-2003 \backslash 0 \backslash \backslash \# P \quad B$ 3LYP/6-31G(D,P) OPT=TIGHT FREQ TEST MAXDISK=19660800 GFINPUT IOP $(6 / 7=3$

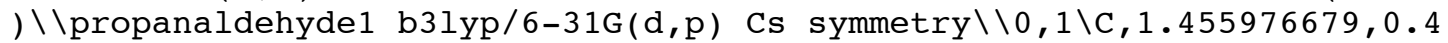
$567123197,0 . \backslash \mathrm{C}, 0.0005575379,0.9157273693,0 . \backslash \mathrm{C},-1.0004953573,-0.2163949$ $766,0 . \backslash 0,-0.71110388,-1.3928085862,0 . \backslash \mathrm{H},-2.0676592602,0.1051065488,0 . \backslash$ $\mathrm{H}, 2.1348533564,1.3138751284,0 . \backslash \mathrm{H}, 1.6709908439,-0.1547706253,0.88019955$ $87 \backslash \mathrm{H}, 1.6709908439,-0.1547706253,-0.8801995587 \backslash \mathrm{H},-0.2282889509,1.548379$ $9944,0.8709942932 \backslash \mathrm{H},-0.2282889509,1.5483799944,-0.8709942932 \backslash \backslash$ Version= $\mathrm{x} 86-\mathrm{Linux}-\mathrm{G} 03 \mathrm{RevB} .03 \backslash$ State $=1-\mathrm{A}^{\prime} \backslash \mathrm{HF}=-193.1537589 \backslash \mathrm{RMSD}=3.392 \mathrm{e}-09 \backslash \mathrm{RMSF}=5$. 205e-06\Dipole $=-0.0135519,1.0017122,0 . \backslash \mathrm{PG}=\mathrm{CS} \quad[\mathrm{SG}(\mathrm{C} 3 \mathrm{H} 2 \mathrm{O} 1), \mathrm{X}(\mathrm{H} 4)] \backslash \backslash @$

\section{6a}

$1 \backslash 1 \backslash G I N C-L C 99 \backslash F O p t \backslash U B 3 L Y P \backslash 6-31 G(d, p) \backslash C 2 H 3 O 1(2) \backslash G M S 501 \backslash 29-0 c t-2003 \backslash 0 \backslash \backslash \#$ P B3LYP/6-31G(D,P) OPT=TIGHT FREQ TEST MAXDISK=19660800 GFINPUT IOP(6/

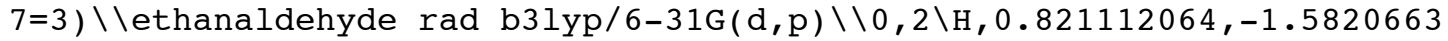
$886,0 . \backslash \mathrm{C}, 1.0594325052,-0.5234723143,0 . \backslash \mathrm{C}, 0.0002604455,0.4294828908,0 . \backslash$ $\mathrm{O},-1.1965369046,0.1075523117,0 . \backslash \mathrm{H}, 0.2938070558,1.4976594553,0 . \backslash \mathrm{H}, 2.099$ $218413,-0.2120750192,0 . \backslash \backslash$ Version=x86-Linux-G03RevB . 03 \State=2-A " $\backslash H F=-1$ $53.175911 \backslash \mathrm{S} 2=0.769392 \backslash \mathrm{S} 2-1=0 . \backslash \mathrm{S} 2 \mathrm{~A}=0.750093 \backslash \mathrm{RMSD}=3.760 \mathrm{e}-09 \backslash \mathrm{RMSF}=1.754 \mathrm{e}-$ $06 \backslash \mathrm{Dipole}=1.0992751,-0.0134545,0 . \backslash \mathrm{PG}=\mathrm{CS} \quad[\mathrm{SG}(\mathrm{C} 2 \mathrm{H} 3 \mathrm{O} 1)] \backslash \backslash @$

\section{TS: $6 \mathbf{a} \rightarrow \mathbf{2 a}$}

$1 \backslash 1 \backslash G I N C-S C 96 \backslash F T S \backslash U B 3 L Y P \backslash 6-31 G(d, p) \backslash C 4 H 9 O 2(2) \backslash G M S 501 \backslash 10-J a n-2005 \backslash 0 \backslash \backslash \#$ B3LYP /6-31G** OPT=(TS, NOEIGEN, CALCFC) FREQ=NORAMAN $\backslash$ lethanradAdo=ethan

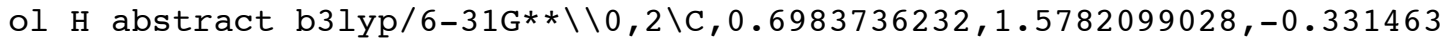
$046 \backslash \mathrm{C}, 1.9443317315,1.4262266047,0.4485422088 \backslash 0,1.9956465608,1.43977916$ $7,1.6665128872 \backslash \mathrm{H}, 2.8641979346,1.2558556346,-0.1543607018 \backslash \mathrm{H}, 0.243592516$ $8,0.3900988789,-0.5561030199 \backslash \mathrm{H}, 0.8307208528,1.9786884729,-1.3382606659$ $\backslash \mathrm{H},-0.1074733456,2.0546866466,0.2284089146 \backslash \mathrm{C},-1.6629214801,-0.95395930$ $77,-0.2596787132 \backslash \mathrm{C},-0.2601557694,-0.92570855,-0.8043024131 \backslash \mathrm{H},-2.282820$ $6291,-0.2065115662,-0.7798897556 \backslash 0,-2.1765673766,-2.2728905465,-0.4574$ $664665 \backslash \mathrm{H}, 0.4599178033,-1.5377861261,-0.2606674433 \backslash \mathrm{H},-0.1740850381,-1.0$ $184529837,-1.8870700962 \backslash \mathrm{H},-1.6512553963,-0.6885117845,0.8086106843 \backslash \mathrm{H},-$ $3.0531968031,-2.3117880355,-0.0516275002 \backslash \backslash$ Version=A164T-G03RevC.02\Sta $t e=2-A \backslash H F=-308.1901051 \backslash S 2=0.759367 \backslash S 2-1=0 . \backslash S 2 A=0.750058 \backslash R M S D=9.523 e-09$ $\backslash \mathrm{RMSF}=3.411 \mathrm{e}-04 \backslash \mathrm{Dipole}=-0.7933172,0.1400688,-0.7998768 \backslash \mathrm{PG}=\mathrm{C} 01[\mathrm{X}(\mathrm{C} 4 \mathrm{H} 90$ $2)] \backslash \backslash$ a

\section{6b}

$1 \backslash 1 \backslash G I N C-L C 8 \backslash F O p t \backslash U B 3 L Y P \backslash 6-31 G(d, p) \backslash C 3 H 501(2) \backslash G M S 501 \backslash 14-N o v-2003 \backslash 0 \backslash \backslash \# P$ B3LYP/6-31G(D,P) OPT=TIGHT FREQ TEST MAXDISK=19660800 GFINPUT IOP(6/7

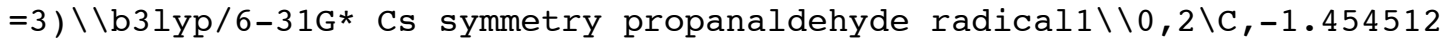
$0739,0.5542699535,0 . \backslash \mathrm{C},-0.0008757162,0.8587150349,0 . \backslash \mathrm{C}, 0.997314086,-0$. $165631831,0 . \backslash 0,0.734988996,-1.3774558849,0 . \backslash \mathrm{H}, 2.050166321,0.1782159238$ $, 0 . \backslash \mathrm{H},-1.6158119697,-0.5250254294,0 . \backslash \mathrm{H},-1.94801777,0.9933683534,0.8778$ $316892 \backslash \mathrm{H},-1.94801777,0.9933683534,-0.8778316892 \backslash \mathrm{H}, 0.3302114454,1.89560$ $09339,0 . \backslash \backslash$ Version=x86-Linux-G03RevB.03 \State $=2-\mathrm{A} " \backslash \mathrm{HF}=-192.5041238 \backslash \mathrm{S} 2=0$ $.765935 \backslash \mathrm{S} 2-1=0 . \backslash \mathrm{S} 2 \mathrm{~A}=0.750073 \backslash \mathrm{RMSD}=4.176 \mathrm{e}-09 \backslash \mathrm{RMSF}=3.419 \mathrm{e}-06 \backslash \mathrm{Dipole}=-0.2$ $862423,1.1330892,0 . \backslash \mathrm{PG}=\mathrm{CS}[\mathrm{SG}(\mathrm{C} 3 \mathrm{H} 3 \mathrm{O} 1), \mathrm{X}(\mathrm{H} 2)] \backslash \backslash @$ 


\section{TS: $6 \mathbf{b} \rightarrow \mathbf{2 b}$}

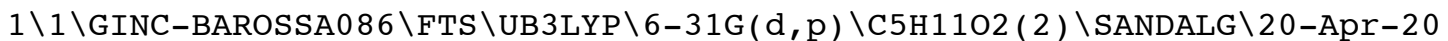
$05 \backslash 0 \backslash \backslash \#$ B3LYP/6-31G(D,P) OPT=(TS, NOEIGEN, CALCFC) FREQ=NORAMAN \propana

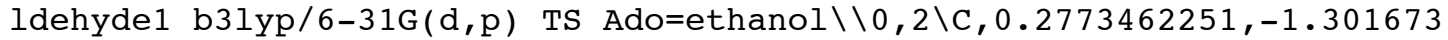
$7277,1.8769652717 \backslash \mathrm{C}, 0.6530081917,-1.4308200365,0.4128171078 \backslash \mathrm{C},-0.40478$ $86945,-1.9374926034,-0.4964478952 \backslash 0,-1.5942331117,-1.9514687194,-0.226$ $7096301 \backslash \mathrm{H},-0.0486499183,-2.2750600139,-1.4954387359 \backslash \mathrm{H}, 1.0283220473,-0$. $7308713673,2.4306379155 \backslash \mathrm{H},-0.6927616729,-0.809958544,1.9809534757 \backslash \mathrm{H}, 0$. $1929864539,-2.2888039996,2.3454718729 \backslash \mathrm{H}, 0.8802198946,-0.2578761968,-0$. $0162680102 \backslash \mathrm{H}, 1.6194673231,-1.9101723306,0.2255480802 \backslash \mathrm{C},-0.1969985153,1$ $.8059425607,-0.4136748807 \backslash \mathrm{C}, 1.150650861,1.1391679511,-0.4045215754 \backslash \mathrm{H},-$ $0.6254212428,1.7995439486,0.6003847044 \backslash 0,-0.0197594451,3.1437968259,-0$ $.8885658149 \backslash \mathrm{H}, 1.6155609572,1.0106217701,-1.3820516411 \backslash \mathrm{H}, 1.8455770427,1$ $.4687284198,0.3673580787 \backslash \mathrm{H},-0.886726249,1.2447482018,-1.0629879717 \backslash \mathrm{H},-$ $0.8919425893,3.5597303946,-0.9222323775 \backslash \backslash$ Version=IA32L-G03RevC.02\Stat $\mathrm{e}=2-\mathrm{A} \backslash \mathrm{HF}=-347.5107862 \backslash \mathrm{S} 2=0.758607 \backslash \mathrm{S} 2-1=0 . \backslash \mathrm{S} 2 \mathrm{~A}=0.750049 \backslash \mathrm{RMSD}=8.047 \mathrm{e}-09 \backslash$ $\mathrm{RMSF}=3.475 \mathrm{e}-06 \backslash \mathrm{Dipole}=0.5412534,0.1483738,0.1544558 \backslash \mathrm{PG}=\mathrm{C} 01 \quad[\mathrm{X}(\mathrm{C} 5 \mathrm{H} 1102)$ ]$\backslash \backslash Q$

\section{$\mathrm{H}_{2} \mathrm{O}$}

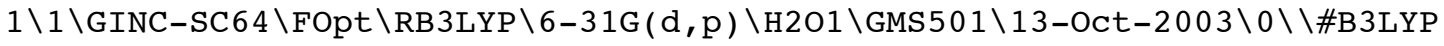
$/ 6-31 \mathrm{G}(\mathrm{D}, \mathrm{P})$ OPT $=(\mathrm{TIGHT}, \mathrm{CALCFC})$ FREQ TEST MAXDISK $=13107200 \backslash \backslash \mathrm{h} 2 \mathrm{ob} 31 \mathrm{yp} / 6$ $-31 \mathrm{G}(\mathrm{d}, \mathrm{p}) \backslash \backslash 0,1 \backslash \mathrm{O}, 0 \ldots, 0.0 .1192085997 \backslash \mathrm{H}, 0 ., 0.7593368728,-0.4768343988 \backslash \mathrm{H}$, 0 . $,-0.7593368728,-0.4768343988 \backslash \backslash$ Version=DEC-AXP-OSF / 1-G03RevB.03 \State $=1-\mathrm{A} 1 \backslash \mathrm{HF}=-76.4197366 \backslash \mathrm{RMSD}=2.851 \mathrm{e}-09 \backslash \mathrm{RMSF}=4.759 \mathrm{e}-08 \backslash \mathrm{Dipole}=0 ., 0 .,-0.803$ $7529 \backslash \mathrm{PG}=\mathrm{C} 02 \mathrm{~V} \quad[\mathrm{C} 2(\mathrm{O} 1), \mathrm{SGV}(\mathrm{H} 2)] \backslash \backslash @$

\section{Ethanol}

$1 \backslash 1 \backslash G I N C-S C 100 \backslash F O p t \backslash R B 3 L Y P \backslash 6-31 G(d, p) \backslash C 2 H 6 O 1 \backslash G M S 501 \backslash 10-D e c-2004 \backslash 0 \backslash \backslash \# B$ 3LYP/6-31G(D,P) OPT=TIGHT FREQ MAXDISK=65536000\\ethanal b3lyp/6-31G(d , p) $\backslash \backslash 0,1 \backslash \mathrm{C}, 0 .,-0.4108199965,-0.3733970491 \backslash \mathrm{C}, 0$. $-0.4842493265,1.1440932$ $505 \backslash \mathrm{H}, 0.8859120452,0.0131303888,1.5496089642 \backslash \mathrm{H},-0.8859120452,0.0131303$ $888,1.5496089642 \backslash \mathrm{H}, 0.8866066022,-0.929781072,-0.7728881235 \backslash \mathrm{H},-0.886606$ $6022,-0.929781072,-0.7728881235 \backslash \mathrm{H}, 0 \ldots,-1.52588461,1.4803969067 \backslash 0,0 ., 0.9$ $645023319,-0.7437595536 \backslash \mathrm{H}, 0 ., 1.0135832592,-1.7079393676 \backslash \backslash$ Version $=\mathrm{Al} 64 \mathrm{~T}$ -G03RevC.02 $\backslash$ State $=1-A^{\prime} \backslash \mathrm{HF}=-155.0462138 \backslash \mathrm{RMSD}=4.710 \mathrm{e}-09 \backslash \mathrm{RMSF}=3.257 \mathrm{e}-06 \backslash \mathrm{D}$ ipole $=0,-0.4410045,-0.4094234 \backslash \mathrm{PG}=\mathrm{CS} \quad[\mathrm{SG}(\mathrm{C} 2 \mathrm{H} 2 \mathrm{O} 1), \mathrm{X}(\mathrm{H} 4)] \backslash \backslash @$

\section{Ethanol Radical}

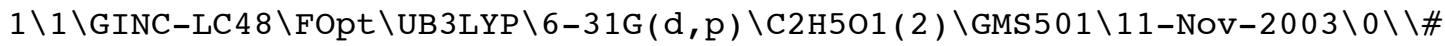
P B3LYP/6-31G(D,P) OPT=(TIGHT,READFC) TEST MAXDISK=26214400FREQ GFINP UT IOP $(6 / 7=3) \backslash \backslash$ ethanal rad b3lyp/6-31G(d,p) C1symmetry oH in $\backslash \backslash 0,2 \backslash \mathrm{C}, 0$ $.0108223448,0.53778621 \overline{7}, 0.0308161344 \backslash \mathrm{C},-1.2355089126,-0.272346092,-0.0$ $129520808 \backslash \mathrm{O}, 1.1940611202,-0.2584734395,0.0446532461 \backslash \mathrm{H}, 0.0212854804,1.2$ $650216324,-0.7989787653 \backslash \mathrm{H}, 0.0640519197,1.1337380569,0.9566178524 \backslash \mathrm{H}, 1.1$ $442897228,-0.8457251149,-0.7217237858 \backslash \mathrm{H},-1.2867468228,-1.19974889,0.54$ $87597442 \backslash \mathrm{H},-2.147249855,0.1218610816,-0.4490853362 \backslash \backslash$ Version=x86-LinuxG03RevB . 03 \State $=2-A \backslash H F=-154.3726787 \backslash S 2=0.753731 \backslash \mathrm{S} 2-1=0 . \backslash \mathrm{S} 2 \mathrm{~A}=0.750009 \backslash$ $\mathrm{RMSD}=3.387 \mathrm{e}-09 \backslash \mathrm{RMSF}=2.634 \mathrm{e}-06 \backslash \mathrm{Dipole}=-0.4661107,-0.0196257,-0.3964782 \backslash$ $\mathrm{PG}=\mathrm{C} 01[\mathrm{X}(\mathrm{C} 2 \mathrm{H} 5 \mathrm{O} 1)] \backslash \backslash @$ 


\section{1a with $\mathrm{K}^{+}$}

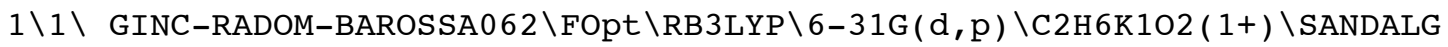

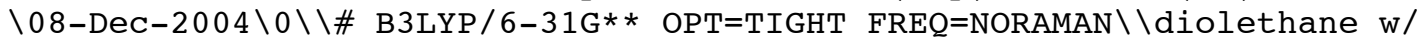
$\mathrm{K}+\mathrm{b} 31 \mathrm{yp} / 6-31 \mathrm{G} * * \backslash \backslash 1,1 \backslash \mathrm{C}, 1.5886906265,0.7007785797,-0.2854 \backslash \mathrm{C}, 1.58866937$ $35,-0.7007914203,0.28543 \backslash 0,0.401801243,1.3904296408,0.1581 \backslash 0,0.4017887$ $57,-1.3904303592,-0.15813 \backslash \mathrm{H}, 1.6132694084,-0.6616914423,1.38206 \backslash \mathrm{H}, 2.488$ $0789115,-1.2175922243,-0.06678 \backslash \mathrm{H}, 1.6133505915,0.6616785577,-1.38204 \backslash \mathrm{H}$, $2.4880910885,1.2175577756,0.06686 \backslash \mathrm{H}, 0.5705879076,-2.3404805101,-0.1173$ $\backslash \mathrm{H}, 0.5705820924,2.3404894899,0.11718 \backslash \mathrm{K},-1.83352,0.0000016392,0 . \backslash \backslash$ Versi on $=x 86-$ Linux-G03RevB. 02 $\backslash$ State $=1-A \backslash H F=-830.0334702 \backslash R M S D=7.513 e-09 \backslash R M S F=$ $3.977 e-06 \backslash \mathrm{Dipole}=-1.4652824,-0.0000006,0.0000278 \backslash \mathrm{PG}=\mathrm{C} 01[\mathrm{X}(\mathrm{C} 2 \mathrm{H} 6 \mathrm{~K} 102)] \backslash$ $\backslash \mathrm{Q}$

\section{TS:1a with $\mathrm{K}^{+} \rightarrow 2 \mathrm{a}$ with $\mathrm{K}^{+}$}

$1 \backslash 1 \backslash$ GINC-RADOM-BAROSSA $119 \backslash \mathrm{FTS} \backslash \mathrm{UB} 3 \mathrm{LYP} \backslash 6-31 \mathrm{G}(\mathrm{d}, \mathrm{p}) \backslash \mathrm{C} 4 \mathrm{H} 11 \mathrm{~K} 103(1+, 2) \backslash$ SANDA

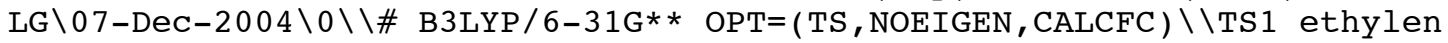
e glycol, with $\mathrm{K}+\mathrm{b} 31 \mathrm{yp} / 6-31 \mathrm{G} * * \backslash \backslash 1,2 \backslash \mathrm{C}, 0.7490348401,-0.5895439628,-1$. $0151406156 \backslash \mathrm{C}, 0.1902498226,-0.2980935693,0.344404268 \backslash 0,2.1857054977,-0$. $3717333114,-1.0187011466 \backslash 0,0.4611321606,1.0464681443,0.7241048833 \backslash \mathrm{H}, 0$. $4943815381,-1.015173051,1.1161276367 \backslash \mathrm{H},-1.1340371275,-0.4141558012,0.2$ $480576162 \backslash \mathrm{H}, 0.2803286748,0.0612862048,-1.7647891579 \backslash \mathrm{H}, 0.5250711752,-1$. $631589325,-1.2666650556 \backslash \mathrm{H},-0.1532102289,1.2927836415,1.4300664367 \backslash \mathrm{H}, 2$. $5677187305,-0.8898765871,-1.7397948116 \backslash \mathrm{K}, 2.9312344583,1.8393764976,0.1$ $872440308 \backslash \mathrm{C},-3.0633380526,-1.7545235637,0.1957793114 \backslash \mathrm{C},-2.5186127795,-$ $0.3460915344,0.2079042863 \backslash \mathrm{H},-2.7160205143,-2.2996417018,1.0880540515 \backslash \mathrm{O}$ $,-4.480006485,-1.641920736,0.1732030393 \backslash \mathrm{H},-2.715654137,0.2239306035,-0$ $.7019142102 \backslash \mathrm{H},-2.7735067602,0.218461977,1.1079796643 \backslash \mathrm{H},-2.6879983678,-$ $2.2958489152,-0.6879477662 \backslash \mathrm{H},-4.8591800613,-2.5313274951,0.1866513019 \backslash$ $\backslash$ Version $=x 86-$ Linux-G03RevB . 02 \State $=2-A \backslash H F=-984.3865317 \backslash \mathrm{S} 2=0.756866 \backslash \mathrm{S} 2$ $-1=0 . \backslash \mathrm{S} 2 \mathrm{~A}=0.750024 \backslash \mathrm{RMSD}=7.625 \mathrm{e}-09 \backslash \mathrm{RMSF}=3.358 \mathrm{e}-06 \backslash \mathrm{Dipole}=3.9494792,1.54$ $01608,0.1228578 \backslash \mathrm{PG}=\mathrm{C} 01 \quad[\mathrm{X}(\mathrm{C} 4 \mathrm{H} 11 \mathrm{~K} 1 \mathrm{O} 3)] \backslash \backslash @$

\section{2a with $\mathrm{K}^{+}$}

$1 \backslash 1 \backslash$ GINC-RADOM-BAROSSA061\FOpt \UB3LYP \6-31G(d,p) \C2H5K102 $(1+, 2) \backslash$ SANDA

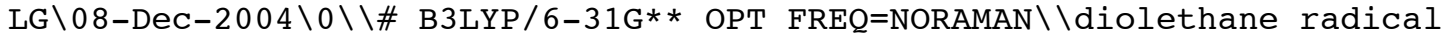
$\mathrm{w} / \mathrm{K}+\mathrm{b} 31 \mathrm{yp} / 6-31 \mathrm{G} * * \backslash \backslash 1,2 \backslash \mathrm{C}, 1.662987127,-0.6669386834,0.1018 \backslash \mathrm{C}, 1.46809$ $48619,0.7253892795,-0.364164 \backslash 0,0.4176512672,-1.4036889205,-0.006624 \backslash 0$, $0.4813167247,1.4463720916,0.285531 \backslash \mathrm{H}, 2.2870277554,1.2851144354,-0.8019$ $29 \backslash \mathrm{H}, 1.9942121311,-0.6888436204,1.154307 \backslash \mathrm{H}, 2.4416512151,-1.1300085352$, $-0.513806 \backslash \mathrm{H}, 0.6459285443,2.394153123,0.178626 \backslash \mathrm{H}, 0.5972814414,-2.318671$ $8863,0.250373 \backslash \mathrm{K},-1.7865439977,-0.0123113401,-0.048665 \backslash \backslash$ Version $=x 86-\mathrm{Lin}$ ux-G03RevB.02 $\backslash$ State $=2-A \backslash H F=-829.3696804 \backslash \mathrm{S} 2=0.753388 \backslash \mathrm{S} 2-1=0 . \backslash \mathrm{S} 2 \mathrm{~A}=0.7500$ $08 \backslash \mathrm{RMSD}=4.583 e-09 \backslash \mathrm{RMSF}=4.113 e-06 \backslash \mathrm{Dipole}=-1.5006977,0.224543,-0.0000982$ $\backslash \mathrm{PG}=\mathrm{C} 01 \quad[\mathrm{X}(\mathrm{C} 2 \mathrm{H} 5 \mathrm{~K} 102)] \backslash \backslash @$

\section{3a with $\mathrm{K}^{+}$}

$1 \backslash 1 \backslash G I N C-S C 14 \backslash F O p t \backslash U B 3 L Y P \backslash 6-31 G(d, p) \backslash C 2 H 5 K 102(1+, 2) \backslash G M S 501 \backslash 20-A u g-2004$ $\backslash 0 \backslash \backslash \# P$ B3LYP/6-31G** OPT=TIGHT FREQ=NORAMAN MAXDISK=39321600 GFINPUT I

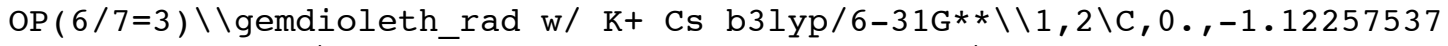
$18,2.2068373114 \backslash \mathrm{H}, 0$. $,-2.0391533451,2.7846152744 \backslash \mathrm{H}, 0$. , $-0.1664619407,2.7$ $198327685 \backslash \mathrm{C}, 0$. $,-1.1470819177,0.7240868335 \backslash 0,1.1028407229,-0.4206699908$ $, 0.1629949648 \backslash 0,-1.1028407229,-0.4206699908,0.1629949648 \backslash \mathrm{H}, 0 .,-2.17370$ $96761,0.3331836311 \backslash \mathrm{H},-1.9244543639,-0.7776273749,0.5278497979 \backslash \mathrm{H}, 1.9244$ $543639,-0.7776273749,0.5278497979 \backslash \mathrm{K}, 0.1 .3833285948,-1.4256208198 \backslash \backslash \mathrm{Ver}$

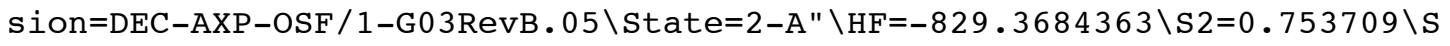
$2-1=0 . \backslash S 2 A=0.750009 \backslash \mathrm{RMSD}=7.195 \mathrm{e}-09 \backslash \mathrm{RMSF}=3.435 \mathrm{e}-06 \backslash \mathrm{Dipole}=0 ., 1.0571694$, $-1.1205881 \backslash \mathrm{PG}=\mathrm{CS} \quad[\mathrm{SG}(\mathrm{C} 2 \mathrm{H} 3 \mathrm{~K} 1), \mathrm{X}(\mathrm{H} 2 \mathrm{O} 2)] \backslash \backslash @$ 


\section{TS:3a with $\mathrm{K}^{+} \rightarrow \mathbf{4 a}$ with $\mathrm{K}^{+}$}

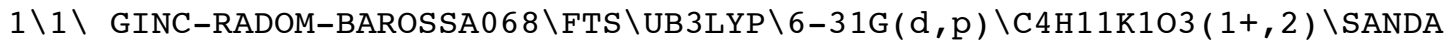

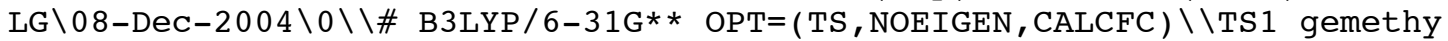
lene glycol, with $\mathrm{K}+\mathrm{b} 31 \mathrm{yp} / 6-31 \mathrm{G} *$ * Ado=ethanol $\backslash \backslash 1,2 \backslash \mathrm{C},-0.8963759555,0$ $.6683790768,-0.6283037825 \backslash \mathrm{C}, 0.6024686221,0.7092495654,-0.6754916423 \backslash 0$, $1.1985926878,0.0434700467,0.4378871816 \backslash 0,1.1374882634,2.0336818619,-0$. $5204431136 \backslash \mathrm{H}, 0.8560991393,-0.8600455077,0.4739430507 \backslash \mathrm{H}, 0.7521082415,2$. $6029121063,-1.2001953263 \backslash \mathrm{H}, 0.9871665829,0.2774701565,-1.6111858911 \backslash \mathrm{H},-$ $1.2499622665,-0.6411997156,-0.5889560843 \backslash \mathrm{H},-1.3829473069,1.0367708759$, $-1.5329868486 \backslash \mathrm{H},-1.3159842152,1.0959337957,0.2837537977 \backslash \mathrm{K}, 2.7325643087$ $, 1.8615574817,1.5694925103 \backslash \mathrm{C},-3.0259451018,-2.104038344,-0.8320371757 \backslash$ $\mathrm{C},-1.5569698553,-1.9584376306,-0.5039774047 \backslash \mathrm{H},-3.2161071536,-1.7472314$ $55,-1.8560866716 \backslash 0,-3.3342099757,-3.4839827173,-0.6913230488 \backslash \mathrm{H},-1.3183$ $608765,-2.2304411913,0.5279215706 \backslash \mathrm{H},-0.8934875147,-2.4425214453,-1.224$ $3972066 \backslash \mathrm{H},-3.6253939621,-1.4831892861,-0.1477543929 \backslash \mathrm{H},-4.2658865945,-3$ $.6143200223,-0.9145218154 \backslash \backslash$ Version=x86-Linux-G03RevB.02 $\backslash$ State $=2-\mathrm{A} \backslash \mathrm{HF}=-$ $984.39389 \backslash \mathrm{S} 2=0.756897 \backslash \mathrm{S} 2-1=0 . \backslash \mathrm{S} 2 \mathrm{~A}=0.750025 \backslash \mathrm{RMSD}=6.683 e-09 \backslash \mathrm{RMSF}=3.601 \mathrm{e}-$ $06 \backslash \mathrm{Dipole}=2.8156513,2.7721022,1.3606359 \backslash \mathrm{PG}=\mathrm{C} 01 \quad[\mathrm{X}(\mathrm{C} 4 \mathrm{H} 11 \mathrm{~K} 1 \mathrm{O} 3)] \backslash \backslash @$

\section{4a with $\mathrm{K}^{+}$}

$1 \backslash 1 \backslash$ GINC-SC14 1 FOpt $\backslash R B 3 L Y P \backslash 6-31 G(d, p) \backslash C 2 H 6 K 102(1+) \backslash G M S 501 \backslash 20-A u g-2004 \backslash 0$ $\backslash \backslash \# P$ B3LYP/6-31G** OPT=TIGHT FREQ=NORAMAN MAXDISK=32768000 GFINPUT IOP $(6 / 7=3) \backslash \backslash$ gemdioleth $\mathrm{w} / \mathrm{K}+\mathrm{Cs} \mathrm{b} 31 \mathrm{yp} / 6-31 \mathrm{G} * * \backslash \backslash 1,1 \backslash \mathrm{C}, 0 .,-1.050644393,2.20$ $59363566 \backslash \mathrm{C}, 0 \ldots,-1.1056676003,0.6900649357 \backslash 0,1.1025888769,-0.4029417662$, $0.1203358325 \backslash \mathrm{O},-1.1025888769,-0.4029417662,0.1203358325 \backslash \mathrm{H}, 1.9258302586$ $,-0.7865741497,0.4508096868 \backslash \mathrm{H},-1.9258302586,-0.7865741497,0.4508096868$ $\backslash \mathrm{H}, 0 .,-2.1438987607,0.3252162095 \backslash \mathrm{H}, 0.8803455201,-1.5600065999,2.610878$ $5571 \backslash \mathrm{H},-0.8803455201,-1.5600065999,2.6108785571 \backslash \mathrm{H}, 0$. , $-0.0118976462,2.5$ $468057544 \backslash \mathrm{K}, 0$. , $1.3807314803,-1.4893041855 \backslash \backslash$ Version=DEC-AXP-OSF $/ 1-\mathrm{G} 03 \mathrm{Re}$ vB.05 \State $=1-A^{\prime} \backslash H F=-830.0437233 \backslash R M S D=3.918 e-09 \backslash R M S F=8.940 e-07 \backslash D i p o l e=$ $0 ., 1.0431341,-1.2653927 \backslash \mathrm{PG}=\mathrm{CS}[\mathrm{SG}(\mathrm{C} 2 \mathrm{H} 2 \mathrm{~K} 1), \mathrm{X}(\mathrm{H} 4 \mathrm{O} 2)] \backslash \backslash \mathrm{a}$

\section{$\mathrm{FH} \cdots 1 \mathrm{a}$}

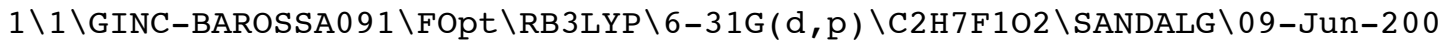
$5 \backslash 0 \backslash \backslash \#$ B3LYP/6-31G** OPT FREQ=NORAMAN \\F ethylene glycol b3lyp/6-31G* $* \backslash \backslash 0,1 \backslash \mathrm{C}, 0.0367773754,0.6675120447,0.1149660119 \backslash 0,0.1643961369,-0.2608$ $523755,-0.9714171408 \backslash \mathrm{H},-0.3149206852,-1.0539634986,-0.6798099577 \backslash \mathrm{H}, 0.3$ $535962623,1.644620344,-0.2578339346 \backslash \mathrm{H}, 0.7033504948,0.3776568358,0.9359$ $506502 \backslash \mathrm{C},-1.4039075062,0.7078866794,0.5856272851 \backslash 0,-1.7352373403,-0.63$ $74430255,0.934859367 \backslash \mathrm{H},-2.0474379998,1.0759952939,-0.2257865831 \backslash \mathrm{H},-2.6$ $932599527,-0.7115861593,1.0197933513 \backslash \mathrm{F}, 2.7150631348,-0.3697212086,-0.5$ $816040784 \backslash \mathrm{H}, 1.8339953557,-0.459114446,-0.913623033 \backslash \mathrm{H},-1.5013812758,1.3$ $878533711,1.4446486203 \backslash \backslash$ Version=IA32L-G03RevC.02 $\backslash$ State=1-A $\backslash H F=-330.704$ $5732 \backslash \mathrm{RMSD}=3.574 \mathrm{e}-09 \backslash \mathrm{RMSF}=1.231 \mathrm{e}-05 \backslash \mathrm{Dipole}=-1.9116771,0.3219796,0.37777$ $67 \backslash \mathrm{PG}=\mathrm{C} 01 \quad[\mathrm{X}(\mathrm{C} 2 \mathrm{H} 7 \mathrm{~F} 1 \mathrm{O} 2)] \backslash \backslash @$ 


\section{TS:FH $\cdots 1 \mathrm{a} \rightarrow \mathrm{FH} \cdots 2 \mathrm{a}$}

$1 \backslash 1 \backslash G I N C-B A R O S S A 095 \backslash F T S \backslash U B 3 L Y P \backslash 6-31 G(d, p) \backslash C 4 H 12 F 103(2) \backslash S A N D A L G \backslash 09-J u n-$ $2005 \backslash 0 \backslash \backslash \#$ B3LYP $/ 6-31 \mathrm{G} * *$ OPT=(TS, NOEIGEN, CALCFC) FREQ=NORAMAN \\TS1 Ado=

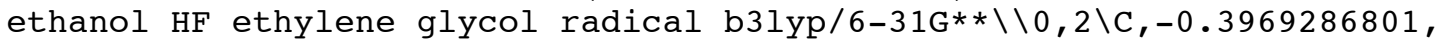
$1.1733211254,0.2162760078 \backslash \mathrm{O},-0.4874955082,2.5435690697,0.6600582339 \backslash \mathrm{H}$, $-0.1856218864,2.5396882738,1.5816998238 \backslash \mathrm{H},-0.5787524434,1.1847401906,-$ $0.8605456917 \backslash \mathrm{H},-1.1841629445,0.5774526103,0.6931562132 \backslash \mathrm{C}, 0.9603739375$, $0.6027551061,0.5230824174 \backslash \mathrm{O}, 1.149040739,0.6542892282,1.9151585543 \backslash \mathrm{H}, 1$. $7697709299,1.0874887836,-0.0389452966 \backslash \mathrm{H}, 2.0774297868,0.4833228617,2.11$ $65762514 \backslash \mathrm{F},-3.0520512295,2.4445977864,0.3696035236 \backslash \mathrm{H},-2.1576514047,2.7$ $116588158,0.5198914299 \backslash \mathrm{H}, 0.9627012614,-0.6471030247,0.1279563125 \backslash \mathrm{C}, 0.5$ $892747132,-2.2065022468,-1.6151392997 \backslash \mathrm{C}, 0.9868367433,-2.0371048833,-0$. $1741251695 \backslash \mathrm{H}, 1.2716530174,-1.6324626125,-2.2635228898 \backslash 0,0.6414804045,-$ $3.6001247974,-1.9339047898 \backslash \mathrm{H}, 0.2815219038,-2.4520131585,0.5472490707 \backslash \mathrm{H}$ $, 2.0179574101,-2.320846845,0.0448586809 \backslash \mathrm{H},-0.4253031544,-1.8067302212$, $-1.7734804136 \backslash \mathrm{H}, 0.3573732232,-3.7032583642,-2.8523849276 \backslash \backslash$ Version=IA32 $\mathrm{L}-\mathrm{G} 03$ RevC. 02 $\backslash \mathrm{State}=2-\mathrm{A} \backslash \mathrm{HF}=-485.0581198 \backslash \mathrm{S} 2=0.756789 \backslash \mathrm{S} 2-1=0 . \backslash \mathrm{S} 2 \mathrm{~A}=0.75002$ $3 \backslash \mathrm{RMSD}=8.434 \mathrm{e}-09 \backslash \mathrm{RMSF}=3.368 \mathrm{e}-06 \backslash \mathrm{Dipole}=1.6241842,-0.1177991,-0.0862628$ $\backslash \mathrm{PG}=\mathrm{C} 01 \quad[\mathrm{X}(\mathrm{C} 4 \mathrm{H} 12 \mathrm{~F} 103)] \backslash \backslash @$

\section{$\mathbf{F H} \cdot \cdot 2 a$}

$1 \backslash 1 \backslash G I N C-B A R O S S A 095 \backslash F O p t \backslash U B 3 L Y P \backslash 6-31 G(d, p) \backslash C 2 H 6 F 102(2) \backslash S A N D A L G \backslash 09-J u n-$ $2005 \backslash 0 \backslash \backslash \#$ B3LYP/6-31G** OPT FREQ=NORAMAN $\backslash$ HF ethylene glycol radical b $31 \mathrm{yp} / 6-31 \mathrm{G} * * \backslash \backslash 0,2 \backslash \mathrm{C}, 0.0737758282,0.4627573442,-0.3092178921 \backslash 0,-0.42327$ $10887,0.0303179741,1.0007880021 \backslash \mathrm{H},-0.0752566763,-0.861677288,1.1452317$ $556 \backslash \mathrm{H},-0.3053736038,1.4786187967,-0.4295766992 \backslash \mathrm{H},-0.3780455462,-0.1685$ $496399,-1.0829518442 \backslash \mathrm{C}, 1.5454862379,0.4402521671,-0.4023900734 \backslash 0,2.081$ $1617932,-0.819644976,-0.5009124774 \backslash \mathrm{H}, 2.1601228394,1.2156492065,0.04965$ $60883 \backslash \mathrm{H}, 3.0445118916,-0.7653641146,-0.4620453953 \backslash \mathrm{F},-2.819620635,0.0019$ $247599,0.0502286001 \backslash \mathrm{H},-2.0480712218,-0.0194408519,0.5982722893 \backslash \backslash$ Versio $\mathrm{n}=\mathrm{IA} 32 \mathrm{~L}-\mathrm{G} 03 \mathrm{RevC} .02 \backslash \mathrm{State}=2-\mathrm{A} \backslash \mathrm{HF}=-330.0431096 \backslash \mathrm{S} 2=0.753327 \backslash \mathrm{S} 2-1=0 . \backslash \mathrm{S} 2 \mathrm{~A}=0$ $.750008 \backslash \mathrm{RMSD}=5.734 \mathrm{e}-09 \backslash \mathrm{RMSF}=8.817 \mathrm{e}-06 \backslash \mathrm{Dipole}=1.974273,-0.1088229,0.126$ $9747 \backslash \mathrm{PG}=\mathrm{C} 01 \quad[\mathrm{X}(\mathrm{C} 2 \mathrm{H} 6 \mathrm{~F} 1 \mathrm{O} 2)] \backslash \backslash @$

\section{$\mathbf{F H} \cdots 3 a$}

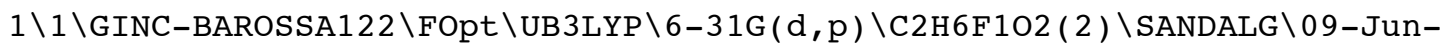
$2005 \backslash 0 \backslash \backslash \#$ B3LYP/6-31G** OPT FREQ=NORAMAN \\HF gemdiol ethylene glycol r

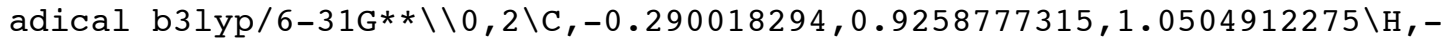
$1.246219436,0.480759455,1.2924700768 \backslash \mathrm{H}, 0.0268406534,1.866640643,1.4822$ $605989 \backslash \mathrm{C}, 0.6265287537,0.1584602088,0.172065595 \backslash 0,1.7942272722,0.898001$ $1048,-0.0299311861 \backslash \mathrm{H}, 0.8323156702,-0.8440032707,0.5761837242 \backslash 0,-0.0067$ $849449,-0.1570399121,-1.1004381781 \backslash \mathrm{H},-0.3187223718,0.6848276915,-1.469$ $2054758 \backslash \mathrm{H}, 2.402201004,0.3515571371,-0.546839501 \backslash \mathrm{F},-1.858659364,-1.5387$ $488333,0.1001873808 \backslash \mathrm{H},-1.28708262,-1.1247593395,-0.5289418721 \backslash \backslash$ Version $=I A 32 L-G 03 R e v C .02 \backslash$ State $=2-A \backslash H F=-330.0464983 \backslash S 2=0.753655 \backslash S 2-1=0 . \backslash S 2 A=0$. $750008 \backslash \mathrm{RMSD}=4.124 \mathrm{e}-09 \backslash \mathrm{RMSF}=2.151 \mathrm{e}-05 \backslash \mathrm{Dipole}=0.7008202,0.5639921,-0.410$ $2151 \backslash \mathrm{PG}=\mathrm{C} 01 \quad[\mathrm{X}(\mathrm{C} 2 \mathrm{H} 6 \mathrm{~F} 102)] \backslash \backslash @$ 


\section{TS:FH $\cdots 3 a \rightarrow F H \cdots 4 a$}

$1 \backslash 1 \backslash G I N C-B A R O S S A 084 \backslash F T S \backslash U B 3 L Y P \backslash 6-31 G(d, p) \backslash C 4 H 12 F 103(2) \backslash S A N D A L G \backslash 09-J u n-$ $2005 \backslash 0 \backslash \backslash \#$ B3LYP $/ 6-31 \mathrm{G} * *$ OPT=(TS, NOEIGEN, CALCFC) FREQ=NORAMAN \\TS2 Ado=

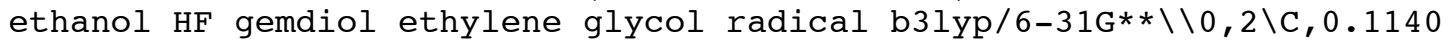
$374518,0.516745907,0.9757030334 \backslash \mathrm{C}, 1.4141707877,0.5888933349,0.22917783$ $12 \backslash 0,2.2691108169,-0.4048951823,0.7151813371 \backslash \mathrm{H}, 1.2562302231,0.51174095$ $89,-0.8537709998 \backslash 0,2.0377360527,1.8938097131,0.359701756 \backslash \mathrm{H}, 2.139351975$ $4,2.063925178,1.3093790649 \backslash \mathrm{H}, 3.0761700268,-0.3868102383,0.182072406 \backslash \mathrm{F}$, $0.0491115642,3.0648001997,-0.8478293948 \backslash \mathrm{H}, 0.8584333725,2.8457989106,-0$ $.4104369371 \backslash \mathrm{H},-0.5850748795,1.2847968672,0.6491350245 \backslash \mathrm{H},-0.4667249379$, $-0.6632018729,0.6613998649 \backslash \mathrm{H}, 0.2394916125,0.4630444064,2.0595263181 \backslash \mathrm{C}$, $-2.1485926723,-1.5667113107,-0.6274507391 \backslash \mathrm{C},-1.020955447,-1.8465754494$ $, 0.3320691614 \backslash \mathrm{H},-1.7639461786,-1.0388067172,-1.5147970861 \backslash 0,-2.7401359$ $221,-2.8159137547,-0.9930306363 \backslash \mathrm{H},-1.3394622439,-2.2635702943,1.288886$ $7731 \backslash \mathrm{H},-0.1873647329,-2.4036786075,-0.0980052059 \backslash \mathrm{H},-2.8890149707,-0.90$ $47877655,-0.150752463 \backslash \mathrm{H},-3.465741646,-2.6297737233,-1.603987582 \backslash \backslash$ Versi on $=I A 32 L-G 03 R e v C .02 \backslash$ State $=2-A \backslash H F=-485.0705573 \backslash \mathrm{S} 2=0.756922 \backslash \mathrm{S} 2-1=0 . \backslash \mathrm{S} 2 \mathrm{~A}=$ $0.750025 \backslash \mathrm{RMSD}=7.864 \mathrm{e}-09 \backslash \mathrm{RMSF}=5.160 \mathrm{e}-06 \backslash \mathrm{Dipole}=0.5105229,-0.0148044,0.2$ $844909 \backslash \mathrm{PG}=\mathrm{C} 01 \quad[\mathrm{X}(\mathrm{C} 4 \mathrm{H} 12 \mathrm{~F} 103)] \backslash \backslash @$

\section{$\mathbf{F H} \cdots 4 a$}

$1 \backslash 1 \backslash G I N C-B A R O S S A 122 \backslash F O p t \backslash R B 3 L Y P \backslash 6-31 G(d, p) \backslash C 2 H 7 F 102 \backslash S A N D A L G \backslash 09-J u n-200$ $5 \backslash 0 \backslash \backslash \#$ B3LYP/6-31G** OPT FREQ=NORAMAN \\HF gemdiol ethylene glycol radi

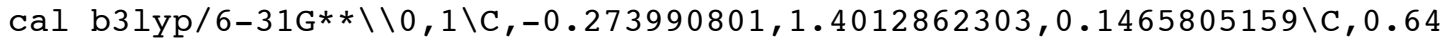
$91249582,0.2066375044,0.0126460715 \backslash 0,1.7922980227,0.6015262867,-0.6907$ $775221 \backslash \mathrm{H}, 0.8931428863,-0.2159715024,0.9952697968 \backslash 0,0.0038751239,-0.893$ $6301975,-0.6603634964 \backslash \mathrm{H},-0.2735753052,-0.5709416963,-1.5318582536 \backslash \mathrm{H}, 2$. $4025269751,-0.1490317473,-0.7078516706 \backslash \mathrm{F},-1.8746459562,-1.1361388123,1$ $.1296725247 \backslash \mathrm{H},-1.2773728507,-1.2276469859,0.4025531707 \backslash \mathrm{H},-1.1885911709$ $, 1.0837373759,0.6493745344 \backslash \mathrm{H}, 0.2094989091,2.1927525287,0.7241437346 \backslash \mathrm{H}$, $-0.5140059539,1.8016402166,-0.8449154106 \backslash \backslash$ Version=IA32L-G03RevC.02\Sta $\mathrm{te}=1-\mathrm{A} \backslash \mathrm{HF}=-330.7229108 \backslash \mathrm{RMSD}=4.537 \mathrm{e}-09 \backslash \mathrm{RMSF}=5.956 \mathrm{e}-06 \backslash \mathrm{Dipole}=0.7372322$, $0.1405204,-0.7079186 \backslash \mathrm{PG}=\mathrm{C} 01 \quad[\mathrm{X}(\mathrm{C} 2 \mathrm{H} 7 \mathrm{~F} 1 \mathrm{O} 2)] \backslash \backslash @$

\section{$\mathrm{NH}_{4}^{+} \cdots \cdot 1 \mathrm{a}$}

$1 \backslash 1 \backslash$ GINC-RADOM-BAROSSA088 \FOpt \RB3LYP \6-31G(d,p) \C2H10N1O2 ( 1+) \SANDAL

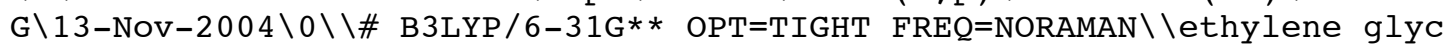
ol, H-bond from NH4+ no donor $\backslash \backslash 1,1 \backslash \mathrm{C},-0.1875029469,0.9850954006,-0.09$ $61614172 \backslash \mathrm{O}, 0.3710431942,-0.3392558329,-0.3040938582 \backslash \mathrm{H},-0.2950119707,-0$ $.9334012432,0.1033991824 \backslash \mathrm{H}, 0.2061066214,1.6445048555,-0.8721422495 \backslash \mathrm{H}, 0$ $.107731967,1.355177971,0.892241024 \backslash \mathrm{C},-1.7013198332,0.8536372436,-0.184$ $8266088 \backslash \mathrm{O},-2.005437254,-0.2515839837,0.6670308883 \backslash \mathrm{H},-2.9184645193,-0.5$ $341729307,0.5275873893 \backslash \mathrm{H}, 1.7838475054,-0.6592009747,-0.120041702 \backslash \mathrm{N}, 2.8$ $670090457,-0.93469663,-0.0710098501 \backslash \mathrm{H}, 3.1595677035,-1.1085114183,0.893$ $3269793 \backslash \mathrm{H}, 3.0346988727,-1.7807996795,-0.6210369332 \backslash \mathrm{H}, 3.442909068,-0.18$ $20829959,-0.4557258743 \backslash \mathrm{H},-2.1784204945,1.7817303614,0.1526209941 \backslash \mathrm{H},-2$. $0039389148,0.6539551318,-1.2207279449 \backslash \backslash$ Version=x86-Linux-G03RevB.02\St ate $=1-\mathrm{A} \backslash \mathrm{HF}=-287.212044 \backslash \mathrm{RMSD}=5.473 \mathrm{e}-09 \backslash \mathrm{RMSF}=3.232 \mathrm{e}-05 \backslash \mathrm{Dipole}=2.841096,-$ $0.8756841,-0.2105857 \backslash \mathrm{PG}=\mathrm{C} 01[\mathrm{X}(\mathrm{C} 2 \mathrm{H} 10 \mathrm{~N} 1 \mathrm{O} 2)] \backslash \backslash @$ 


\section{TS: $\mathrm{NH}_{4}^{+} \cdots \cdot 1 \mathrm{a} \rightarrow \mathrm{NH}_{4}^{+} \cdot \cdots \cdot 2 \mathrm{a}$}

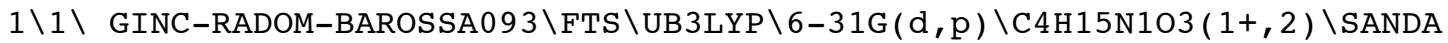

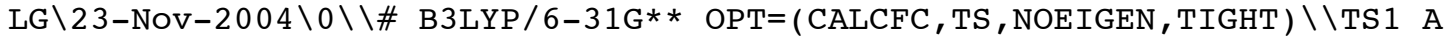

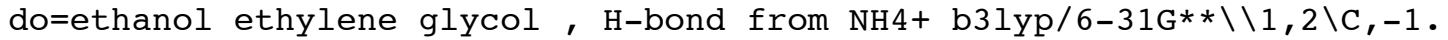
$246968157,-0.36364368,-0.0542250828 \backslash 0,-2.4532993663,0.3802631082,-0.43$ $00718402 \backslash \mathrm{H},-2.1557066104,1.310743042,-0.4593837279 \backslash \mathrm{H},-1.3580662719,-1$. $3815075613,-0.4315734156 \backslash \mathrm{H},-1.1716110833,-0.3774070013,1.0394748154 \backslash \mathrm{C}$, $-0.0522130956,0.3244424904,-0.6591128075 \backslash 0,-0.1804139911,1.6882201818$, $-0.329926195 \backslash \mathrm{H}, 0.4564349089,2.2135859639,-0.8316419997 \backslash \mathrm{H},-3.7568401896$ $, 0.1127955,0.1477322753 \backslash \mathrm{N},-4.7975686786,-0.1126579489,0.5063304937 \backslash \mathrm{H},-$ $4.9984933591,-1.109987297,0.4013476907 \backslash \mathrm{H},-4.9129877651,0.1414728526,1$. $4900030023 \backslash \mathrm{H},-5.4789708714,0.4113944485,-0.0480421418 \backslash \mathrm{H}, 1.0222500983,-$ $0.1891916513,-0.0750026488 \backslash \mathrm{H}, 0.0624906522,0.1461154833,-1.7345196578 \backslash \mathrm{C}$ $, 3.3529072103,-0.4754248211,-0.2378143827 \backslash \mathrm{C}, 2.1376038675,-0.6350002689$ $, 0.6423685702 \backslash \mathrm{H}, 3.4532029443,0.5765955451,-0.5531879122 \backslash 0,4.4769415702$ $,-0.8943646368,0.5265946307 \backslash \mathrm{H}, 1.895498731,-1.6702816544,0.8902093037 \backslash \mathrm{H}$ , $2.1267328093,0.0162599446,1.5181738343 \backslash \mathrm{H}, 3.2388068892,-1.0819518162$, $1.1505500577 \backslash \mathrm{H}, 5.2664352139,-0.8252217037,-0.0274233624 \backslash \backslash$ Version $=\mathrm{x} 86-\mathrm{L}$ inux-G03RevB.02 $\backslash$ State $=2-A \backslash H F=-441.5653624 \backslash S 2=0.7567 \backslash \mathrm{S} 2-1=0 . \backslash \mathrm{S} 2 \mathrm{~A}=0.7500$ $23 \backslash \mathrm{RMSD}=5.577 \mathrm{e}-09 \backslash \mathrm{RMSF}=1.246 \mathrm{e}-06 \backslash \mathrm{Dipole}=-6.057514,0.2881555,-0.2066247$ $\backslash \mathrm{PG}=\mathrm{C} 01 \quad[\mathrm{X}(\mathrm{C} 4 \mathrm{H} 15 \mathrm{~N} 103)] \backslash \backslash \mathrm{Q}$

\section{$\mathrm{NH}_{4}{ }^{+\cdots \cdot 2 a}$}

$1 \backslash 1 \backslash$ GINC-RADOM-BAROSSA108\Freq \UB3LYP \6-31G (d,p) \C2H9N102 $(1+, 2) \backslash$ SANDA LG \16-Nov-2004\0\\\# B3LYP/6-31G** FREQ=NORAMAN \lethylene glycol radica 1 , H-bond from NH4+ no donor $\backslash \backslash 1,2 \backslash \mathrm{C},-0.4470888864,0.6951241136,0.28230$ $69545 \backslash 0,0.5577491136,-0.2645158864,-0.3077690455 \backslash \mathrm{H}, 0.1507521136,-1.144$ $4978864,-0.2822140455 \backslash \mathrm{H},-0.0702038864,1.6829921136,0.0171269545 \backslash \mathrm{H},-0.4$ $201908864,0.5542231136,1.3680269545 \backslash \mathrm{C},-1.7933808864,0.4791731136,-0.24$ $51190455 \backslash \mathrm{O},-2.4132028864,-0.6304748864,0.2363369545 \backslash \mathrm{H},-2.1749858864,0$. $9644561136,-1.1364450455 \backslash \mathrm{H},-3.2930098864,-0.7285318864,-0.1540650455 \backslash \mathrm{H}$ $, 1.9884291136,-0.1911488864,-0.0497980455 \backslash \mathrm{N}, 3.1039301136,-0.1103368864$ $, 0.0508129545 \backslash \mathrm{H}, 3.3879941136,-0.1182358864,1.0331019545 \backslash \mathrm{H}, 3.5595661136$ $,-0.8922028864,-0.4260190455 \backslash \mathrm{H}, 3.4305871136,0.7594471136,-0.3770760455$ $\backslash \backslash$ Version $=x 86-$ Linux-G03RevB. 02 \State $=2-A \backslash H F=-286.5501474 \backslash \mathrm{S} 2=0.75337 \backslash \mathrm{S} 2$ $-1=0 . \backslash \mathrm{S} 2 \mathrm{~A}=0.750008 \backslash \mathrm{RMSD}=7.042 \mathrm{e}-09 \backslash \mathrm{RMSF}=5.857 \mathrm{e}-06 \backslash \mathrm{Dipole}=3.0891901,-0.1$ $642144,0.1454772 \backslash \mathrm{PG}=\mathrm{C} 01[\mathrm{X}(\mathrm{C} 2 \mathrm{H} 9 \mathrm{~N} 1 \mathrm{O} 2)] \backslash \backslash @$

\section{$\mathrm{NH}_{4}^{+} \cdot \cdots 3 \mathrm{a}$}

$1 \backslash 1 \backslash$ GINC-RADOM-BAROSSA092\Freq \UB3LYP \6-31G(d,p) \C2H9N1O2 $(1+, 2) \backslash$ SANDA LG \16-NOv-2004\0\\\# B3LYP/6-31G** FREQ=NORAMAN \\gemdiolethane radical, $\mathrm{H}$-bond from NH4+ freq no donor $\backslash \backslash 1,2 \backslash \mathrm{C}, 1.2458410455,1.3970041591,-0.07$ $20019773 \backslash \mathrm{H}, 0.6693450455,2.2376691591,0.2935830227 \backslash \mathrm{H}, 2.0771440455,1.561$ $2111591,-0.7469559773 \backslash \mathrm{C}, 0.9114850455,0.0192201591,0.3439730227 \backslash 0,1.955$ $4130455,-0.8185378409,0.0321530227 \backslash \mathrm{H}, 0.5818210455,-0.0494538409,1.3886$ $920227 \backslash \mathrm{O},-0.3193929545,-0.4634788409,-0.3872099773 \backslash \mathrm{H},-0.0667529545,-0$. $5103468409,-1.3247499773 \backslash \mathrm{H}, 1.8412900455,-1.6751068409,0.4681930227 \backslash \mathrm{H},-$ $1.7575359545,-0.1249668409,-0.1025469773 \backslash \mathrm{N},-2.8335919545,0.0195611591$, $0.1031200227 \backslash \mathrm{H},-3.3755009545,-0.7252098409,-0.3429529773 \backslash \mathrm{H},-3.00987295$ $45,-0.0128048409,1.1105100227 \backslash \mathrm{H},-3.1569109545,0.9208681591,-0.25698297$ $73 \backslash \backslash$ Version=x86-Linux-G03RevB. 02 \State=2-A \HF=-286.5442943 \S2=0.753694 $\backslash \mathrm{S} 2-1=0 . \backslash \mathrm{S} 2 \mathrm{~A}=0.750009 \backslash \mathrm{RMSD}=2.050 \mathrm{e}-09 \backslash \mathrm{RMSF}=7.217 \mathrm{e}-06 \backslash \mathrm{Dipole}=-3.6979171$, $-0.0436759,0.2240452 \backslash \mathrm{PG}=\mathrm{C} 01[\mathrm{X}(\mathrm{C} 2 \mathrm{H} 9 \mathrm{~N} 1 \mathrm{O} 2)] \backslash \backslash @$ 


\section{TS: $\mathrm{NH}_{4}^{+} \cdots \cdot 3 \mathrm{a} \rightarrow \mathrm{NH}_{4}^{+} \cdots \cdot 4 \mathrm{a}$}

$1 \backslash 1 \backslash$ GINC-RADOM-BAROSSA065 \FTS \UB3LYP \6-31G $(\mathrm{d}, \mathrm{p}) \backslash \mathrm{C} 4 \mathrm{H} 15 \mathrm{~N} 103(1+, 2) \backslash$ SANDA

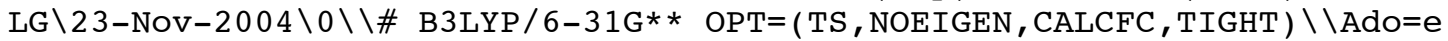

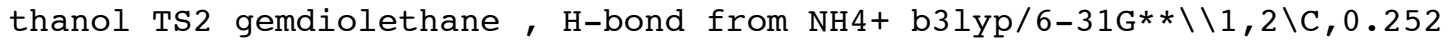
$166174,-0.4462517453,-0.4824747059 \backslash \mathrm{H}, 0.6147679374,-1.4440496109,-0.236$ $0370241 \backslash \mathrm{H},-0.9743463478,-0.3759977406,0.107296978 \backslash \mathrm{C}, 1.0611840476,0.666$ $0251448,0.0990211024 \backslash 0,0.5117347248,1.8728877531,-0.2755008205 \backslash \mathrm{H}, 1.201$ $2235488,0.5666587596,1.18255751 \backslash 0,2.481637035,0.6327235238,-0.39684772$ $26 \backslash \mathrm{H}, 2.4378358243,0.862447338,-1.3401822694 \backslash \mathrm{H}, 0.8939576977,2.590799055$ $7,0.2492468824 \backslash \mathrm{H}, 3.5213548693,-0.3472103856,0.0027724128 \backslash \mathrm{N}, 4.357891414$ $,-1.0038892457,0.3293363359 \backslash \mathrm{H}, 5.2519828665,-0.6040855462,0.0333705699 \backslash$ $\mathrm{H}, 4.3645801613,-1.086833305,1.3489710832 \backslash \mathrm{H}, 4.2680504804,-1.9394490461$, $-0.0740224849 \backslash \mathrm{H}, 0.0379803965,-0.3172737391,-1.5444580103 \backslash \mathrm{C},-3.22855588$ $68,-0.6327450554,-0.3107900292 \backslash C,-2.1698338227,-0.2754037109,0.7060663$ $287 \backslash \mathrm{H},-3.0554288329,-1.6522158321,-0.6912955791 \backslash 0,-4.4813082683,-0.532$ $0549772,0.3547344293 \backslash \mathrm{H},-2.1961175491,0.7660485491,1.0306652424 \backslash \mathrm{H},-2.10$ $60760209,-0.9710647796,1.5449912869 \backslash \mathrm{H},-3.1697995664,0.0552349511,-1.16$ $87271572 \backslash \mathrm{H},-5.1814763672,-0.7339821447,-0.2805270579 \backslash \backslash$ Version $=x 86-\mathrm{Linu}$ $\mathrm{x}-\mathrm{G} 03$ RevB. $02 \backslash$ State $=2-\mathrm{A} \backslash \mathrm{HF}=-441.5703904 \backslash \mathrm{S} 2=0.756695 \backslash \mathrm{S} 2-1=0 . \backslash \mathrm{S} 2 \mathrm{~A}=0.75002$ $4 \backslash \mathrm{RMSD}=9.188 \mathrm{e}-09 \backslash \mathrm{RMSF}=1.509 \mathrm{e}-06 \backslash \mathrm{Dipole}=6.2735238,-1.1962261,-0.0347526$ $\backslash P G=C 01[X(C 4 \mathrm{H} 15 \mathrm{~N} 103)] \backslash \backslash @$

\section{$\mathrm{NH}_{4}{ }^{+} \cdot .4 \mathrm{a}$}

$1 \backslash 1 \backslash$ GINC-RADOM-BAROSSA $115 \backslash$ Freq $\backslash$ RB $3 L Y P \backslash 6-31 \mathrm{G}(\mathrm{d}, \mathrm{p}) \backslash \mathrm{C} 2 \mathrm{H} 10 \mathrm{~N} 1 \mathrm{O} 2(1+) \backslash$ SANDAL

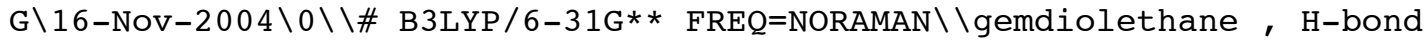
from NH4+ no donor $\backslash \backslash 1,1 \backslash \mathrm{C}, 1.2026600222,1.375911,-0.0877800667 \backslash \mathrm{H}, 0.3617$ $430222,2.050435,0.0924229333 \backslash \mathrm{H}, 2.0649660222,1.736022,0.4777489333 \backslash \mathrm{C}, 0$. $8696810222,-0.036742,0.3404659333 \backslash 0,1.9244240222,-0.861572,0.013898933$ $3 \backslash \mathrm{H}, 0.5839940222,-0.096888,1.3978539333 \backslash \mathrm{O},-0.3441289778,-0.526139,-0.3$ $559990667 \backslash \mathrm{H},-0.0783499778,-0.691943,-1.2756110667 \backslash \mathrm{H}, 1.8447260222,-1.70$ $9361,0.4742249333 \backslash \mathrm{H},-1.7823389778,-0.146761,-0.0931480667 \backslash \mathrm{N},-2.8498319$ $778,0.036466,0.1096089333 \backslash \mathrm{H},-3.4009809778,-0.781607,-0.1631460667 \backslash \mathrm{H},-2$ $.9970789778,0.207303,1.1077049333 \backslash \mathrm{H},-3.1861409778,0.848479,-0.41406206$ $67 \backslash \mathrm{H}, 1.4618780222,1.395733,-1.1505650667 \backslash \backslash$ Version $=x 86-$ Linux-G03RevB. 02 $\backslash$ State $=1-\mathrm{A} \backslash \mathrm{HF}=-287.2208033 \backslash \mathrm{RMSD}=3.652 \mathrm{e}-09 \backslash \mathrm{RMSF}=2.609 \mathrm{e}-06 \backslash \mathrm{Dipole}=-3.734$ $4122,-0.1050587,0.2604773 \backslash \mathrm{PG}=\mathrm{C} 01[\mathrm{X}(\mathrm{C} 2 \mathrm{H} 10 \mathrm{~N} 1 \mathrm{O} 2)] \backslash \backslash @$

\section{$1 \mathrm{a} \cdot \cdot \mathrm{NH}_{3}$}


B3LYP/6-31G** OPT FREQ=NORAMAN \lethylene glycol donating to nh3 $\backslash \backslash 0,1 \backslash \mathrm{C}$ $,-1.842140987,-0.3645430658,-0.09751 \backslash 0,-1.9164310375,1.0485299315,-0.1$ $67849 \backslash \mathrm{H},-1.1761860479,1.339540958,0.389498 \backslash \mathrm{H},-2.4538299723,-0.77580808$ $77,-0.908386 \backslash \mathrm{H},-2.2493829736,-0.7395510804,0.85648 \backslash \mathrm{C},-0.3946769706,-0$. $8221560141,-0.231886 \backslash 0,0.3435250053,-0.1485119877,0.773476 \backslash \mathrm{H}, 1.2684560$ $012,-0.0347889547,0.456627 \backslash \mathrm{N}, 2.9906309914,0.2399641069,-0.194946 \backslash \mathrm{H}, 3.5$ $142920221,-0.6172208744,-0.354102 \backslash \mathrm{H}, 3.0096939727,0.7642391075,-1.06625$ $\backslash \mathrm{H}, 3.516441972,0.7830171256,0.485779 \backslash \mathrm{H},-0.3315109314,-1.9188730118,-0$. $126596 \backslash \mathrm{H},-0.02823698,-0.560243001,-1.237064 \backslash \backslash$ Version=Al64T-G03RevC.02 $\backslash$ State $=1-\mathrm{A} \backslash \mathrm{HF}=-286.8318021 \backslash \mathrm{RMSD}=8.876 \mathrm{e}-09 \backslash \mathrm{RMSF}=1.599 \mathrm{e}-06 \backslash \mathrm{Dipole}=2.06210$ $88,-0.2728346,-0.4616417 \backslash \mathrm{PG}=\mathrm{C} 01[\mathrm{X}(\mathrm{C} 2 \mathrm{H} 9 \mathrm{~N} 1 \mathrm{O} 2)] \backslash \backslash @$ 


\section{TS:1a $\cdots \mathrm{NH}_{3} \rightarrow \mathbf{2 a} \cdots \mathrm{NH}_{3}$}

$1 \backslash 1 \backslash G I N C-S C 108 \backslash F T S \backslash U B 3 L Y P \backslash 6-31 G(d, p) \backslash C 4 H 14 N 103(2) \backslash G M S 501 \backslash 29-M a r-2005 \backslash 0$ \\\# B3LYP/6-31G** OPT=(CALCFC,TS, NOEIGEN, TIGHT) FREQ=NORAMAN \\TS1 Ado= ethanol ethylene glycol donating to $\mathrm{nh} 3 \backslash \backslash 0,2 \backslash \mathrm{C}, 1.7562100155,-0.9742156$ $937,0.5015133571 \backslash \mathrm{O}, 3.061094015,-0.584280493,0.9161728489 \backslash \mathrm{H}, 3.304872197$ $6,0.1287825125,0.3070811161 \backslash \mathrm{H}, 1.3515900355,-1.6309114668,1.2785829376 \backslash$ $\mathrm{H}, 1.7943284192,-1.544598173,-0.4406980906 \backslash \mathrm{C}, 0.861823796,0.2316603889,0$ $.3112638217 \backslash 0,1.4219444944,1.0620913474,-0.652384735 \backslash \mathrm{H}, 1.0317361823,1$. $9670743022,-0.5653946988 \backslash \mathrm{N}, 0.2692909501,3.6136970663,-0.4059995951 \backslash \mathrm{H},-$ $0.7439032904,3.5650610863,-0.4809107964 \backslash \mathrm{H}, 0.4796006247,4.0952430326,0$. $4648050906 \backslash \mathrm{H}, 0.5912819924,4.2146236666,-1.1610132597 \backslash \mathrm{H},-0.2909349547,-$ $0.1948597998,-0.086669638 \backslash \mathrm{H}, 0.6494675606,0.7496790224,1.2597995854 \backslash \mathrm{C},-$ $2.1923473841,-1.6181566081,0.2582660272 \backslash \mathrm{C},-1.5807350613,-0.5873447279$, $-0.6452666014 \backslash \mathrm{H},-2.2803219376,-1.2172567988,1.2818760837 \backslash \mathrm{O},-3.48553234$ $37,-1.9766307029,-0.2504797746 \backslash \mathrm{H},-1.3091994803,-0.947088232,-1.6383918$ $341 \backslash \mathrm{H},-2.1242914818,0.3586517287,-0.6826384394 \backslash \mathrm{H},-1.539307719,-2.50425$ $56638,0.3119872515 \backslash \mathrm{H},-3.8497123215,-2.6571260489,0.3324555152 \backslash \backslash$ Version $=\mathrm{Al} 64 \mathrm{~T}-\mathrm{G} 03 \mathrm{RevC} .02 \backslash \mathrm{State}=2-\mathrm{A} \backslash \mathrm{HF}=-441.1878782 \backslash \mathrm{S} 2=0.756687 \backslash \mathrm{S} 2-1=0 . \backslash \mathrm{S} 2 \mathrm{~A}=0$. $750023 \backslash \mathrm{RMSD}=4.530 \mathrm{e}-09 \backslash \mathrm{RMSF}=1.173 \mathrm{e}-06 \backslash \mathrm{Dipole}=-0.6120357,1.7434431,0.285$ $8081 \backslash \mathrm{PG}=\mathrm{C} 01 \quad[\mathrm{X}(\mathrm{C} 4 \mathrm{H} 14 \mathrm{~N} 1 \mathrm{O})] \backslash \backslash @$

\section{$2 \mathrm{a} \cdot \cdots \mathrm{NH}_{3}$}

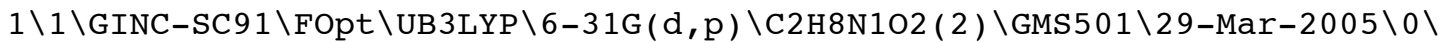
$\backslash \#$ B3LYP/6-31G** OPT FREQ=NORAMAN \ethylene glycol radical donating to $\mathrm{nh} 3 \backslash \backslash 0,2 \backslash \mathrm{C},-1.7690789668,-0.5354271096,0.030123 \backslash 0,-2.3067470475,0.766$ $765857,-0.28963 \backslash \mathrm{H},-1.7527760863,1.3930738914,0.196633 \backslash \mathrm{H},-2.2945909231$, $-1.2414351422,-0.619541 \backslash \mathrm{H},-2.0076559509,-0.7918041244,1.074684 \backslash \mathrm{C},-0.30$ $24869605,-0.6372460187,-0.179448 \backslash 0,0.4417769953,0.0759930274,0.713481 \backslash$ $\mathrm{H}, 0.0997180458,-0.7389679938,-1.189634 \backslash \mathrm{H}, 1.3810989929,0.1139780856,0.3$ $98661 \backslash \mathrm{N}, 3.0837479905,0.1540761911,-0.201479 \backslash \mathrm{H}, 3.4455420485,-0.78216778$ $64,-0.365771 \backslash \mathrm{H}, 3.1998759582,0.6737861983,-1.067971 \backslash \mathrm{H}, 3.6917119635,0.58$ $89662288,0.48843 \backslash \backslash$ Version $=\mathrm{A} 164 \mathrm{~T}-\mathrm{G} 03$ RevC.02 $\backslash$ State $=2-\mathrm{A} \backslash \mathrm{HF}=-286.1730168 \backslash \mathrm{S}$ $2=0.753279 \backslash \mathrm{S} 2-1=0 . \backslash \mathrm{S} 2 \mathrm{~A}=0.750008 \backslash \mathrm{RMSD}=7.966 \mathrm{e}-09 \backslash \mathrm{RMSF}=1.540 \mathrm{e}-06 \backslash \mathrm{Dipole}=2$ $.3195555,-0.0005613,-0.289578 \backslash \mathrm{PG}=\mathrm{C} 01 \quad[\mathrm{X}(\mathrm{C} 2 \mathrm{H} 8 \mathrm{~N} 1 \mathrm{O} 2)] \backslash \backslash @$

\section{$3 \mathrm{a} \cdot \cdots \mathrm{NH}_{3}$}

$1 \backslash 1 \backslash G I N C-S C 91 \backslash F O p t \backslash U B 3 L Y P \backslash 6-31 G(d, p) \backslash C 2 H 8 N 1 O 2(2) \backslash G M S 501 \backslash 29-M a r-2005 \backslash 0 \backslash$ $\backslash \#$ B3LYP/6-31G** OPT FREQ=NORAMAN \gemdiolethane radical donating to $\mathrm{n}$ $\mathrm{h} 3 \backslash \backslash 0,2 \backslash \mathrm{C}, 2.1889880031,-0.1728609602,0.125897 \backslash \mathrm{H}, 2.8644549902,0.5381160$ $52,0.586816 \backslash \mathrm{H}, 2.5779030175,-0.9626509532,-0.505598 \backslash \mathrm{C}, 0.7203460005,-0.0$ $301929869,0.315544 \backslash 0,0.08139102,-1.1016039985,-0.297139 \backslash \mathrm{H}, 0.462921999$, $0.0550200084,1.385009 \backslash 0,0.2567929774,1.2432040047,-0.203656 \backslash \mathrm{H}, 0.499052$ $9775,1.2398870091,-1.142538 \backslash \mathrm{H},-0.884035982,-0.9929440161,-0.11965 \backslash \mathrm{N},-2$ $.5000469988,-0.0670570454,0.114512 \backslash \mathrm{H},-3.0263249997,-0.015029055,0.9826$ $23 \backslash \mathrm{H},-3.176116998,-0.1117900577,-0.643344 \backslash \mathrm{H},-1.9790000146,0.8043089641$ , $0.012811 \backslash \backslash$ Version $=\mathrm{A} 164 \mathrm{~T}-\mathrm{G} 03$ RevC $.02 \backslash$ State $=2-\mathrm{A} \backslash \mathrm{HF}=-286.1750794 \backslash \mathrm{S} 2=0.753$ $804 \backslash \mathrm{S} 2-1=0 . \backslash \mathrm{S} 2 \mathrm{~A}=0.750009 \backslash \mathrm{RMSD}=3.855 \mathrm{e}-09 \backslash \mathrm{RMSF}=2.566 \mathrm{e}-06 \backslash \mathrm{Dipole}=-0.76151$ $14,0.4883508,-0.0567 \backslash \mathrm{PG}=\mathrm{C} 01 \quad[\mathrm{X}(\mathrm{C} 2 \mathrm{H} 8 \mathrm{~N} 1 \mathrm{O} 2)] \backslash \backslash @$ 


\section{TS:3a $\cdots \mathrm{NH}_{3} \rightarrow 4 \mathrm{a} \cdots \mathrm{NH}_{3}$}

$1 \backslash 1 \backslash G I N C-S C 123 \backslash F T S \backslash U B 3 L Y P \backslash 6-31 G(d, p) \backslash C 4 H 14 N 103(2) \backslash G M S 501 \backslash 29-M a r-2005 \backslash 0$ \\\# B3LYP/6-31G** OPT=(TS, NOEIGEN, CALCFC, TIGHT) FREQ=NORAMAN \\Ado=etha


$26956,-1.4430421468,0.433355094 \backslash C, 1.2982200318,0.0010348121,0.36053265$ $04 \backslash 0,0.6583158277,0.5973164206,-0.7260617809 \backslash \mathrm{H}, 1.0783480742,0.51882181$ $63,1.306302813 \backslash 0,2.7311776181,0.1252011269,0.2554925378 \backslash \mathrm{H}, 2.9677282003$ $,-0.3072776086,-0.5793699472 \backslash \mathrm{H}, 0.9166150799,1.5514565969,-0.7160650978$ $\backslash \mathrm{N}, 2.0249653522,3.0365991008,-0.4649589363 \backslash \mathrm{H}, 1.8753007193,3.7485996702$ $, 0.2449984095 \backslash \mathrm{H}, 2.3817043577,3.508528756,-1.2916714406 \backslash \mathrm{H}, 2.7557140246$, $2.4100030487,-0.1272164952 \backslash \mathrm{H}, 1.3125345463,-1.9881520107,1.2708978091 \backslash \mathrm{H}$ $,-0.4538849521,-1.4638693658,0.6563027217 \backslash \mathrm{H}, 0.9897838575,-1.9695459547$ $,-0.5178055561 \backslash \mathrm{C},-2.4118767086,-0.8238257575,-0.3570954022 \backslash \mathrm{C},-1.791282$ $2684,-1.4449972463,0.8666107456 \backslash \mathrm{H},-2.1858544902,-1.4396025148,-1.24230$ $73757 \backslash \mathrm{O},-3.8273398335,-0.7358390354,-0.146210496 \backslash \mathrm{H},-1.9088193355,-0.84$ $98267547,1.7744876803 \backslash \mathrm{H},-2.0553392934,-2.4916489695,1.0303780541 \backslash \mathrm{H},-1$. $9688817308,0.1660029323,-0.5310113158 \backslash \mathrm{H},-4.2043379242,-0.2781294128,-0$ $.9093883191 \backslash \backslash$ Version $=A 164 \mathrm{~T}-\mathrm{G} 03$ RevC $.02 \backslash$ State $=2-\mathrm{A} \backslash \mathrm{HF}=-441.2007847 \backslash \mathrm{S} 2=0.7$ $56966 \backslash \mathrm{S} 2-1=0 . \backslash \mathrm{S} 2 \mathrm{~A}=0.750026 \backslash \mathrm{RMSD}=7.800 \mathrm{e}-09 \backslash \mathrm{RMSF}=4.446 \mathrm{e}-06 \backslash \mathrm{Dipole}=1.0144$ $75,0.9958442,-0.5157406 \backslash \mathrm{PG}=\mathrm{C} 01 \quad[\mathrm{X}(\mathrm{C} 4 \mathrm{H} 14 \mathrm{~N} 103)] \backslash \backslash @$

\section{$4 \mathrm{a} \cdot \cdot \cdot \mathrm{NH}_{3}$}

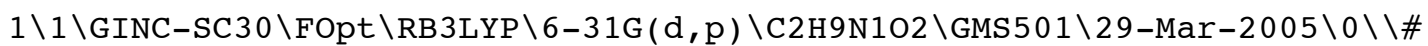
B3LYP $/ 6-31 \mathrm{G} *$ * OPT=TIGHT FREQ=NORAMAN $\backslash$ gemdiolethane donating to nh3 $\backslash \backslash 0$ $, 1 \backslash \mathrm{C},-1.7827077013,1.2411192848,0.0787766793 \backslash \mathrm{C},-0.5462805166,0.3821458$ $546,0.2912287816 \backslash 0,0.4877644497,0.9023652367,-0.4828277255 \backslash \mathrm{H},-0.283686$ $4067,0.342831526,1.3596978987 \backslash 0,-0.8046189085,-0.994523304,-0.02928624$ $97 \backslash \mathrm{H},-0.9833320981,-1.0083315751,-0.98191938 \backslash \mathrm{H}, 1.2890593344,0.36332860$ $52,-0.2799097434 \backslash \mathrm{N}, 2.2590847335,-1.1985017176,0.1535590206 \backslash \mathrm{H}, 2.7384042$ $46,-1.3560455003,1.0360490335 \backslash \mathrm{H}, 2.8248446338,-1.626668369,-0.574438068$ $7 \backslash \mathrm{H}, 1.3704784604,-1.6986360918,0.1889784993 \backslash \mathrm{H},-2.6289235047,0.84047927$ $52,0.6423662145 \backslash \mathrm{H},-1.5918423824,2.2694507397,0.3974012757 \backslash \mathrm{H},-2.0398304$ $399,1.2607771155,-0.9862598379 \backslash \backslash$ Version=Al64 T-G03RevC.02 $\backslash$ State $=1-\mathrm{A} \backslash \mathrm{HF}=$ $-286.8510018 \backslash \mathrm{RMSD}=3.917 \mathrm{e}-09 \backslash \mathrm{RMSF}=3.297 \mathrm{e}-06 \backslash \mathrm{Dipole}=0.4548094,-0.7823534$ , $0.0593456 \backslash \mathrm{PG}=\mathrm{C} 01 \quad[\mathrm{X}(\mathrm{C} 2 \mathrm{H} 9 \mathrm{~N} 1 \mathrm{O} 2)] \backslash \backslash @$

\section{$1 \mathrm{a} \cdot \cdot \mathrm{OCHO}^{-}$}

$1 \backslash 1 \backslash$ GINC-RADOM-BAROSSA105\FOpt \RB3LYP \6-31G(d,p) \C3H7O4 (1-) \SANDALG 2

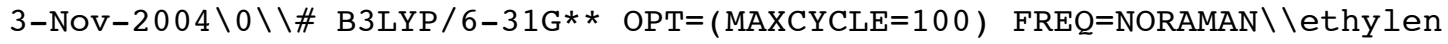
e glycol and HCCO- b3lyp/6-31G** \\-1,1\C, $-2.3429270001,0.4433429994,-0$ $.220338 \backslash \mathrm{O},-2.304702,0.1034179994,1.154895 \backslash \mathrm{H},-1.6319569998,-0.607494000$ $4,1.14796 \backslash \mathrm{H},-2.6311650004,1.4997449993,-0.318924 \backslash \mathrm{H},-3.108003,-0.158452$ $0008,-0.748201 \backslash C,-0.968156,0.1903519998,-0.85082 \backslash 0,-0.5515669997,-1.09$ $41090001,-0.432683 \backslash \mathrm{H}, 0.4391190003,-1.0361489999,-0.200228 \backslash \mathrm{O}, 1.97063900$ $03,-0.9952709995,0.187804 \backslash \mathrm{C}, 2.525434,0.1466810006,0.219071 \backslash 0,2.0510589$ $997,1.2749290005,-0.008112 \backslash \mathrm{H}, 3.619697,0.1052410009,0.497695 \backslash \mathrm{H},-1.05827$ $90001,0.2573769997,-1.952558 \backslash \mathrm{H},-0.2389620002,0.9457349999,-0.528458 \backslash \backslash \mathrm{V}$ ersion $=x 86-L$ inux-G03RevB. 02 \State $=1-A \backslash H F=-419.4873324 \backslash \mathrm{RMSD}=4.797 \mathrm{e}-09 \backslash \mathrm{R}$ $\mathrm{MSF}=1.431 \mathrm{e}-06 \backslash \mathrm{Dipole}=-1.2873278,0.0544308,-0.4365933 \backslash \mathrm{PG}=\mathrm{C} 01 \quad[\mathrm{X}(\mathrm{C} 3 \mathrm{H} 704)$ ]$\backslash \backslash$ 


\section{TS: $1 \mathrm{a} \cdots \mathrm{OCHO}^{-} \rightarrow 2 \mathrm{a} \cdots \mathrm{OCHO}^{-}$}

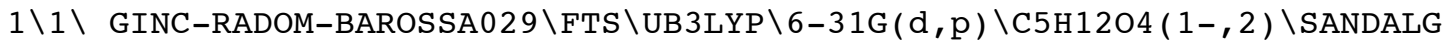
$\backslash 24-N o v-2004 \backslash 0 \backslash \backslash \#$ B3LYP/6-31G** OPT=(TS,NOEIGEN, CALCFC) \\TS1 ado=ethan

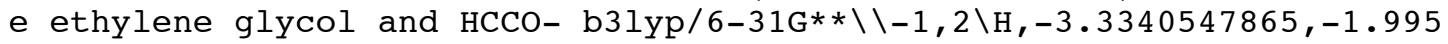
$9580812,-0.1786618934 \backslash \mathrm{C},-2.331300543,-1.4807151022,-0.1683590509 \backslash 0,-1$. $5466138275,-1.9222292737,0.7278287837 \backslash 0,-2.1506114313,-0.5866618208,-1$ $.0163418626 \backslash \mathrm{H},-0.182337618,-1.2351355241,0.8292553354 \backslash 0,0.7557349008,-$ $0.8192313675,0.9338092333 \backslash \mathrm{C}, 2.4570629702,0.1924995003,-0.3460633696 \backslash 0$, $3.0238831071,-1.1070330888,-0.5239790987 \backslash \mathrm{H}, 2.4715628148,-1.6454410857$, $0.0703538929 \backslash \mathrm{H}, 2.6565844051,0.7809526001,-1.250800514 \backslash \mathrm{H}, 2.9449671604,0$ $.7058552561,0.5023728871 \backslash \mathrm{C}, 0.9650953162,0.0903109649,-0.0860916954 \backslash \mathrm{H}, 0$ $.5490877452,1.2671129965,0.2543940516 \backslash \mathrm{H}, 0.3600847841,-0.1235067252,-0$. $9766085288 \backslash \mathrm{C},-1.2513062052,2.7099104054,-0.2759572494 \backslash \mathrm{C},-0.1220706267$, $2.4880342776,0.7033379361 \backslash \mathrm{H},-1.7966718473,1.7754709635,-0.4533489523 \backslash \mathrm{H}$ $,-0.8724965274,3.0547934945,-1.2467605413 \backslash \mathrm{H},-1.9740299912,3.4683414384$ $, 0.0707268151 \backslash \mathrm{H}, 0.640191469,3.2713534922,0.7490747118 \backslash \mathrm{H},-0.4269150691$, $2.1571653055,1.6982668662 \backslash \backslash$ Version=x86-Linux-G03RevB . 02 $\backslash$ State $=2-\mathrm{A} \backslash \mathrm{HF}=-$ $498.642214 \backslash \mathrm{S} 2=0.756744 \backslash \mathrm{S} 2-1=0 . \backslash \mathrm{S} 2 \mathrm{~A}=0.750024 \backslash \mathrm{RMSD}=6.876 \mathrm{e}-09 \backslash \mathrm{RMSF}=4.159 \mathrm{e}$ $-06 \backslash \mathrm{Dipole}=0.8447357,1.3024531,0.0959332 \backslash \mathrm{PG}=\mathrm{C} 01 \quad[\mathrm{X}(\mathrm{C} 5 \mathrm{H} 12 \mathrm{O} 4)] \backslash \backslash @$

\section{$2 \mathrm{a} \cdot \cdots \mathrm{OCHO}^{-}$}

$1 \backslash 1 \backslash$ GINC-RADOM-BAROSSA005 $\backslash$ FOpt \UB3LYP \6-31G(d,p) \C3H6O4 $(1-, 2) \backslash$ SANDALG

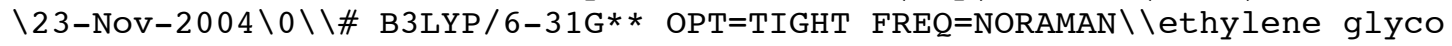

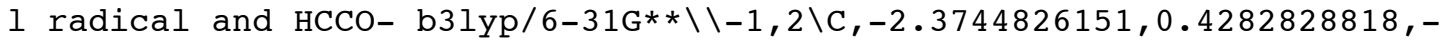
$0.4275321407 \backslash \mathrm{O},-2.853899194,0.0189775116,0.8844453666 \backslash \mathrm{H},-2.2210206537$, $-0.6753007135,1.1229688115 \backslash \mathrm{H},-2.680422891,1.471951936,-0.5706017877 \backslash \mathrm{H}$, $-2.9006399231,-0.1829222846,-1.1852879831 \backslash \mathrm{C},-0.9038336468,0.2868946425$ $,-0.5734415958 \backslash \mathrm{O},-0.4598358241,-0.9730165771,-0.3437238118 \backslash \mathrm{H},-0.207193$ $4009,1.0976113804,-0.362522751 \backslash \mathrm{H}, 0.5701231552,-0.952968454,-0.18696112$ $36 \backslash 0,2.0471430164,-1.0132136224,0.0547570261 \backslash \mathrm{C}, 2.6479399658,0.10324231$ $03,0.1794686163 \backslash 0,2.2000018106,1.2599289045,0.122253014 \backslash \mathrm{H}, 3.7541330196$ $,-0.0103006049,0.3695827962 \backslash \backslash$ Version=x86-Linux-G03RevB.02 $\backslash$ State $=2-A \backslash H F$ $=-418.8318667 \backslash \mathrm{S} 2=0.753317 \backslash \mathrm{S} 2-1=0 . \backslash \mathrm{S} 2 \mathrm{~A}=0.750008 \backslash \mathrm{RMSD}=9.746 \mathrm{e}-09 \backslash \mathrm{RMSF}=3.1$ $74 \mathrm{e}-06 \backslash \mathrm{Dipole}=-1.0581871,-0.1240452,-0.1332757 \backslash \mathrm{PG}=\mathrm{C} 01[\mathrm{X}(\mathrm{C} 3 \mathrm{H} 6 \mathrm{O} 4)] \backslash \backslash \mathrm{a}$

\section{$3 \mathrm{a} \cdots \mathrm{OCHO}^{-}$}

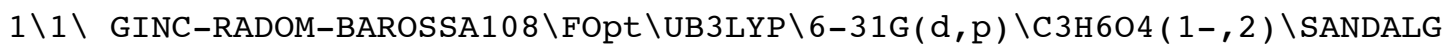
$\backslash 22-N O v-2004 \backslash 0 \backslash \backslash \#$ B3LYP/6-31G** OPT=TIGHT FREQ=NORAMAN \\gemethdiol rad ical and HCCO- b3lyp/6-31G**\\-1,2\C,2.794105906,-0.0004677201,0.30271 $12477 \backslash \mathrm{H}, 3.3271933362,0.9375538827,0.4143713846 \backslash \mathrm{H}, 3.3269086488,-0.93869$ $38608,0.4140140385 \backslash \mathrm{C}, 1.3033770469,-0.0002259596,0.2175944958 \backslash 0,0.88515$ $5235,-1.1813188478,-0.4214867314 \backslash \mathrm{H}, 0.890761247,-0.0005076681,1.2558101$ $476 \backslash 0,0.885502874,1.1813861209,-0.4207321067 \backslash \mathrm{H},-0.1004725181,1.2134350$ $765,-0.2927957151 \backslash \mathrm{H},-0.1008120224,-1.2131934634,-0.2935023499 \backslash 0,-1.795$ $8821163,-1.1398747868,0.0615726983 \backslash \mathrm{C},-2.321100837,0.0002559332,0.13768$ $36465 \backslash \mathrm{O},-1.7955259739,1.1402612439,0.06206999 \backslash \mathrm{H},-3.4358715371,0.000402$ $6705,0.3027753519 \backslash \backslash$ Version=x86-Linux-G03RevB. 02 \State $=2-\mathrm{A} \backslash \mathrm{HF}=-418.8374$ $698 \backslash \mathrm{S} 2=0.75375 \backslash \mathrm{S} 2-1=0 . \backslash \mathrm{S} 2 \mathrm{~A}=0.750009 \backslash \mathrm{RMSD}=5.472 \mathrm{e}-09 \backslash \mathrm{RMSF}=3.306 \mathrm{e}-06 \backslash \mathrm{Dipo}$ $l e=0.8063426,-0.0002391,0.5363761 \backslash P G=C 01[X(C 3 H 604)] \backslash \backslash @$ 


\section{TS: $3 \mathrm{a} \cdots \mathrm{OCHO}^{-} \rightarrow \mathbf{4 a} \cdots \mathrm{OCHO}^{-}$}

$1 \backslash 1 \backslash$ GINC-RADOM-BAROSSA065 $\backslash F T S \backslash U B 3 L Y P \backslash 6-31 G(d, p) \backslash C 5 H 1204(1-, 2) \backslash \operatorname{SANDALG}$ $\backslash 13-D e C-2004 \backslash 0 \backslash \backslash \#$ B3LYP/6-31G** OPT=(TS, NOEIGEN, CALCFC) \TS2 ado=ethan

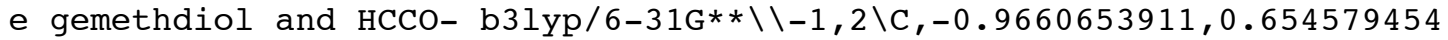
$4,1.7735768409 \backslash \mathrm{C},-0.4993367374,0.5725819316,0.3360861808 \backslash 0,0.779655177$ $5,-0.0160524008,0.3257700246 \backslash \mathrm{H},-1.1998021395,-0.0667507922,-0.24006099$ $51 \backslash 0,-0.5170021395,1.8712559946,-0.1984776278 \backslash \mathrm{H},-0.3541538633,1.764355$ $6791,-1.1771139747 \backslash \mathrm{H}, 1.0113859574,-0.1472970139,-0.6351409415 \backslash 0,1.2493$ $042722,-0.3583165507,-2.3203819652 \backslash \mathrm{C}, 0.6872993295,0.4596343149,-3.0928$ $45174 \backslash \mathrm{O},-0.086080435,1.4168920909,-2.8317308539 \backslash \mathrm{H}, 0.9069043515,0.31003$ $27603,-4.186939527 \backslash \mathrm{H},-1.9780089964,1.0503437604,1.8881013753 \backslash \mathrm{H},-1.0198$ $892751,-0.6158743712,2.2573191529 \backslash \mathrm{H},-0.2440883328,1.1618832033,2.41843$ $63506 \backslash \mathrm{C}, 0.4563271813,-2.3144976186,2.7099892961 \backslash \mathrm{C},-0.9997399385,-1.905$ $668511,2.6472736087 \backslash \mathrm{H}, 0.9703506095,-1.8243093179,3.5454019099 \backslash \mathrm{H}, 0.9605$ $823061,-2.0080273554,1.7885001888 \backslash \mathrm{H}, 0.5812606607,-3.4005392207,2.84117$ $23444 \backslash \mathrm{H},-1.5708851896,-2.399157191,1.8565286051 \backslash \mathrm{H},-1.5415777537,-1.934$ $6706413,3.5978743747 \backslash \backslash$ Version=x86-Linux-G03RevB .02 $\backslash$ State $=2-A \backslash H F=-498.6$ $585637 \backslash \mathrm{S} 2=0.756996 \backslash \mathrm{S} 2-1=0 . \backslash \mathrm{S} 2 \mathrm{~A}=0.750025 \backslash \mathrm{RMSD}=2.638 \mathrm{e}-09 \backslash \mathrm{RMSF}=3.843 e-06 \backslash$ Dipole $=-0.6900003,-1.1318067,1.9269647 \backslash \mathrm{PG}=\mathrm{C} 01 \quad[\mathrm{X}(\mathrm{C} 5 \mathrm{H} 12 \mathrm{O} 4)] \backslash \backslash @$

\section{$4 \mathrm{a} \cdot \cdot \mathrm{OCHO}^{-}$}

$1 \backslash 1 \backslash$ GINC-RADOM-BAROSSA030

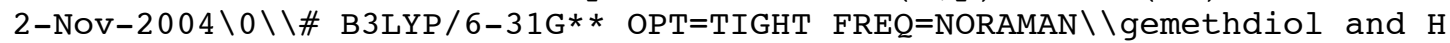

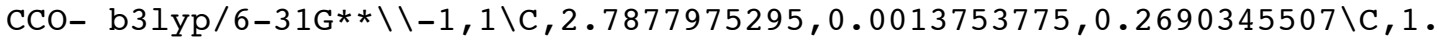
$263357219,0.002184563,0.2768405624 \backslash 0,0.833578876,-1.1800808017,-0.3463$ $045155 \backslash \mathrm{H}, 0.9042460026,0.0128959321,1.3281379711 \backslash 0,0.8347107912,1.17213$ $89143,-0.3698807964 \backslash \mathrm{H},-0.1553653913,1.1937578346,-0.2754802829 \backslash \mathrm{H},-0.15$ $65191654,-1.1988511553,-0.2514921823 \backslash 0,-1.8662825892,-1.1385585803,0.0$ $26550903 \backslash \mathrm{C},-2.3940796615,0.0018606933,0.0739160563 \backslash 0,-1.8652244358,1.1$ $406037306,0.0036177208 \backslash \mathrm{H},-3.5138640648,0.0036712231,0.2022653549 \backslash \mathrm{H}, 3.1$ $765387952,0.8999043949,0.759116745 \backslash \mathrm{H}, 3.1756777042,-0.8875491944,0.7769$ $874585 \backslash \mathrm{H}, 3.1325744605,-0.0091789415,-0.770148576 \backslash \backslash$ Version=x86-Linux-G0 3 RevB . 02 \State $=1-A \backslash H F=-419.5145161 \backslash$ RMSD=5 .396e-09 $\backslash$ RMSF=2 . 501e-06 $\backslash \mathrm{Dipol}$ $e=0.9641985,0.0042201,0.4651049 \backslash \mathrm{PG}=\mathrm{C} 01 \quad[\mathrm{X}(\mathrm{C} 3 \mathrm{H} 704)] \backslash \backslash \mathrm{a}$

\section{$\mathrm{FH} \cdots 1 \mathrm{a} \cdots \mathrm{OCHO}^{-}$}

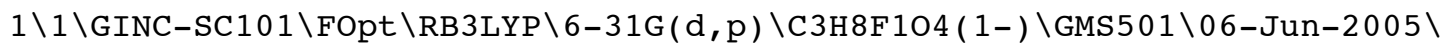
$0 \backslash \backslash \#$ B3LYP/6-31G** OPT FREQ=NORAMAN $\backslash \mathrm{HF}$ ethylene glycol radical and HC

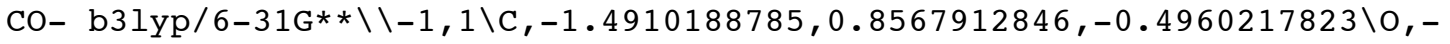
$1.6507606819,0.1349140134,0.7337050757 \backslash \mathrm{H},-1.0301660246,-0.6192586597,0$ $.564742981 \backslash \mathrm{H},-1.6239859946,1.9263275095,-0.2961813097 \backslash \mathrm{H},-2.2725168933$, $0.5356362182,-1.2000493863 \backslash \mathrm{C},-0.0868591553,0.5460715954,-1.0356033383 \backslash$ $0,0.1320248911,-0.8353012354,-0.821904533 \backslash \mathrm{H}, 0.6803995378,1.1312530214$, $-0.5115830146 \backslash \mathrm{H}, 1.0861338309,-0.9457811067,-0.4446844317 \backslash 0,2.480359174$ $9,-1.1527521486,0.1442705462 \backslash \mathrm{C}, 3.1176623301,-0.1076719979,0.4902478766$ $\backslash 0,2.7834708071,1.0887394792,0.4223637254 \backslash \mathrm{H}, 4.1363941479,-0.3282488089$ $, 0.9185890066 \backslash \mathrm{F},-4.1060231056,-0.4317218749,0.5315965522 \backslash \mathrm{H},-3.17342616$ $4,-0.2786365929,0.7135006096 \backslash \mathrm{H},-0.0480837976,0.8082591314,-2.107918474$ $7 \backslash \backslash$ Version=Al64T-G03RevC.02 \State $=1-A \backslash H F=-519.94592 \backslash$ RMSD $=7.467 e-09 \backslash$ RMS $\mathrm{F}=3.517 \mathrm{e}-05 \backslash \mathrm{Dipole}=-1.0098726,0.4515285,-0.4797482 \backslash \mathrm{PG}=\mathrm{C} 01 \quad[\mathrm{X}(\mathrm{C} 3 \mathrm{H} 8 \mathrm{~F} 104)$ ]$\backslash \backslash Q$ 


\section{TS: $\mathrm{FH} \cdots 1 \mathrm{a} \cdots \mathrm{OCHO}^{-} \rightarrow \mathrm{FH}^{\prime} \cdots 2 \mathrm{a} \cdots \mathrm{OCHO}^{-}$}

$1 \backslash 1 \backslash G I N C-S C 121 \backslash F T S \backslash U B 3 L Y P \backslash 6-31 G(d, p) \backslash C 5 H 13 F 104(1-, 2) \backslash G M S 501 \backslash 07-J u n-200$ $5 \backslash 0 \backslash \backslash \#$ B3LYP/6-31G** OPT=(TS,NOEIGEN, CALCFC) FREQ=NORAMAN \\TS1 Ado=eth

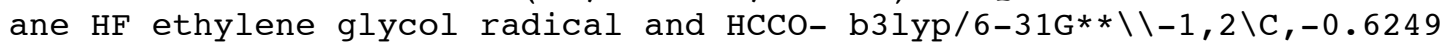
$297021,-1.5393406804,0.9051876553 \backslash 0,-2.0046924003,-1.2101250532,1.1962$ $02215 \backslash \mathrm{H},-1.9544032076,-0.229675796,1.2269451016 \backslash \mathrm{H},-0.6102402456,-2.483$ $6637105,0.3514112593 \backslash \mathrm{H},-0.0967517752,-1.684436293,1.8580754636 \backslash \mathrm{C},-0.01$ $79667691,-0.4030432291,0.1034824676 \backslash 0,-0.3990005757,0.7887352172,0.693$ $6917424 \backslash \mathrm{H},-0.2285569178,-0.4310021564,-0.9725302805 \backslash \mathrm{H},-0.4319017048,1$. $5382708876,-0.0326878569 \backslash 0,-0.5278430764,2.6447027283,-1.0204620429 \backslash \mathrm{C}$, $-0.1245062734,2.3770467303,-2.1971348889 \backslash 0,0.3029286586,1.3056994947,-$ $2.6645490193 \backslash \mathrm{H},-0.1775671455,3.256336153,-2.8969342289 \backslash \mathrm{F},-2.2279507197$ $,-2.3508146691,3.4500055955 \backslash \mathrm{H},-2.2537963526,-1.8810234289,2.6130070239$ $\backslash \mathrm{H}, 1.2710459692,-0.5391279238,0.1378876601 \backslash \mathrm{C}, 3.0701195147,-0.421174029$ $,-1.3838594464 \backslash \mathrm{C}, 2.7195897969,-0.4439439467,0.0856718666 \backslash \mathrm{H}, 2.420091634$ $9,0.276446502,-1.9248162371 \backslash \mathrm{H}, 3.1101218162,-1.285235517,0.6650474884 \backslash \mathrm{H}$ ,2.8434794109,0.5058582925,0.6094936954\H,2.9383071969,-1.410690817,-1 $.839660762 \backslash \mathrm{H}, 4.1167475463,-0.1240923378,-1.5644377739 \backslash \backslash$ Version=Al64T-G 03 RevC.02 $\backslash$ State $=2-A \backslash H F=-599.0993655 \backslash S 2=0.756744 \backslash \mathrm{S} 2-1=0 . \backslash \mathrm{S} 2 \mathrm{~A}=0.750024 \backslash \mathrm{R}$ $M S D=2.926 e-09 \backslash R M S F=3.347 e-06 \backslash D i p o l e=0.7348197,-0.9855591,0.2498954 \backslash P G=$ $\mathrm{C} 01[\mathrm{X}(\mathrm{C} 5 \mathrm{H} 13 \mathrm{~F} 104)] \backslash \backslash @$

\section{$\mathrm{FH} \cdot \cdot 2 \mathrm{a} \cdot \cdots \mathrm{OCHO}^{-}$}

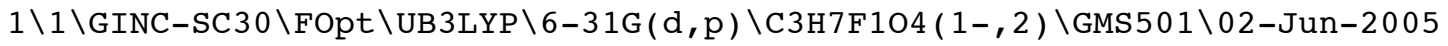
$\backslash 0 \backslash \backslash \#$ B3LYP/6-31G** OPT FREQ=NORAMAN $\backslash$ HF ethylene glycol radical and $\mathrm{H}$

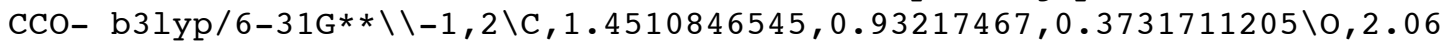
$5641011,0.2178310719,-0.7748974173 \backslash \mathrm{H}, 1.5362624883,-0.5988009766,-0.795$ $8984495 \backslash \mathrm{H}, 1.598447316,2.0012005536,0.1945987624 \backslash \mathrm{H}, 2.0419421656,0.63579$ $4587,1.2535567364 \backslash \mathrm{C}, 0.0216570331,0.5833793283,0.4981190053 \backslash 0,-0.197234$ $736,-0.7495344116,0.5180282609 \backslash \mathrm{H},-0.786298613,1.2168662143,0.136038883$ $9 \backslash \mathrm{H},-1.2223348223,-0.9439984675,0.3252543629 \backslash \mathrm{O},-2.5982888166,-1.282335$ $9808,0.0604983904 \backslash \mathrm{C},-3.3566350708,-0.317867681,-0.2924311821 \backslash 0,-3.0921$ $677832,0.8855920552,-0.4355087873 \backslash \mathrm{H},-4.414166631,-0.6440910045,-0.4953$ $361196 \backslash \mathrm{F}, 4.3980943221,-0.1516681617,0.1396524163 \backslash \mathrm{H}, 3.5430620947,-0.060$ $4972325,-0.2932031589 \backslash \backslash$ Version=Al64T-G03RevC . 02 \State=2-A \HF=-519.2906 $616 \backslash \mathrm{S} 2=0.753312 \backslash \mathrm{S} 2-1=0 . \backslash \mathrm{S} 2 \mathrm{~A}=0.750008 \backslash \mathrm{RMSD}=4.133 \mathrm{e}-09 \backslash \mathrm{RMSF}=3.043 e-05 \backslash \mathrm{Dip}$ ole $=0.9605556,0.2137283,-0.0653089 \backslash \mathrm{PG}=\mathrm{C} 01[\mathrm{X}(\mathrm{C} 3 \mathrm{H} 7 \mathrm{~F} 104)] \backslash \backslash @$

\section{$\mathrm{FH} \cdot \cdot 3 \mathrm{a} \cdots \cdot \mathrm{OCHO}^{-}$}

$1 \backslash 1 \backslash G I N C-S C 122 \backslash F O p t \backslash U B 3 L Y P \backslash 6-31 G(d, p) \backslash C 3 H 7 F 104(1-, 2) \backslash G M S 501 \backslash 02-J u n-200$ $5 \backslash 0 \backslash \backslash \#$ B3LYP/6-31G** OPT FREQ=NORAMAN \\HF gemdiol ethylene glycol radi cal and HCCO- b3lyp/6-31G**\\-1,2\C,-1.3558570457,1.3338181102,0.99781 $1997 \backslash \mathrm{H},-2.3731780065,1.0345993615,1.2207820371 \backslash \mathrm{H},-0.9152610789,2.18860$ $13897,1.499233636 \backslash \mathrm{C},-0.4781748774,0.4543737891,0.1726141654 \backslash 0,0.356466$ $7132,1.1837167083,-0.6370425932 \backslash \mathrm{H}, 0.0946189679,-0.2670455897,0.7802185$ $688 \backslash 0,-1.3535812563,-0.4056106491,-0.683611174 \backslash \mathrm{H},-1.2773478,0.04455123$ $59,-1.5387017011 \backslash \mathrm{H}, 1.237879725,0.6535605144,-0.7507031855 \backslash 0,2.54538061$ $98,-0.1036247388,-0.9262892286 \backslash \mathrm{C}, 2.8523681793,-0.8441355055,0.06179242$ $92 \backslash 0,2.210671449,-1.091878321,1.0980936013 \backslash \mathrm{H}, 3.8598210751,-1.337075660$ $6,-0.0446724178 \backslash \mathrm{F},-3.7720077889,-0.4612264249,0.0948114391 \backslash \mathrm{H},-2.859980$ $5246,-0.4913157859,-0.2219762819 \backslash \backslash$ Version=Al64T-G03RevC.02 $\backslash$ State $=2-A \backslash H$ $\mathrm{F}=-519.2842058 \backslash \mathrm{S} 2=0.753637 \backslash \mathrm{S} 2-1=0 . \backslash \mathrm{S} 2 \mathrm{~A}=0.750008 \backslash \mathrm{RMSD}=9.322 \mathrm{e}-09 \backslash \mathrm{RMSF}=2$. $009 e-05 \backslash \mathrm{Dipole}=-0.9980279,0.5700859,-0.3045327 \backslash \mathrm{PG}=\mathrm{C} 01[\mathrm{X}(\mathrm{C} 3 \mathrm{H} 7 \mathrm{~F} 104)] \backslash \backslash \mathrm{a}$ 


\section{TS:FH $\cdots 3 \mathrm{a} \cdots \cdot \mathrm{OCHO}^{-} \rightarrow \mathrm{FH}^{\prime} \cdot 4 \mathrm{a} \cdot \cdots \mathrm{OCHO}^{-}$}

$1 \backslash 1 \backslash G I N C-S C 18 \backslash$ Freq $\backslash U B 3 L Y P \backslash 6-31 G(d, p) \backslash C 5 H 13 F 104(1-, 2) \backslash G M S 501 \backslash 07-J u n-200$ $5 \backslash 0 \backslash \backslash \#$ B3LYP/6-31G** FREQ=NORAMAN \\TS2 Ado=ethanol HF gemdiol ethylene glycol radical and $\mathrm{HCCO}-\mathrm{b} 31 \mathrm{yp} / 6-31 \mathrm{G} * * \backslash \backslash-1,2 \backslash \mathrm{C}, 1.3471710476,0.7849118$ $81,0.4876200476 \backslash C, 0.6858590476,-0.528537119,0.1566550476 \backslash 0,-0.01823295$ $24,-1.010867119,1.2264140476 \backslash \mathrm{H}, 0.0670910476,-0.452995119,-0.7452579524$ $\backslash 0,1.7490440476,-1.525772119,-0.1953409524 \backslash \mathrm{H}, 1.7823290476,-2.057522119$ $, 0.6144350476 \backslash \mathrm{H},-0.8919109524,-1.440505119,0.8758290476 \backslash 0,-2.170480952$ $4,-2.057336119,0.3498270476 \backslash \mathrm{C},-2.7700669524,-1.343386119,-0.5144599524$ $\backslash 0,-2.4177429524,-0.271907119,-1.0429879524 \backslash \mathrm{H},-3.7614959524,-1.7678561$ $19,-0.8377999524 \backslash \mathrm{F}, 3.9800030476,-0.407331119,-0.7186819524 \backslash \mathrm{H}, 3.1499440$ $476,-0.861644119,-0.5403289524 \backslash \mathrm{H}, 2.0315710476,1.156589881,-0.275680952$ $4 \backslash \mathrm{H}, 0.2981190476,1.658350881,0.5446420476 \backslash \mathrm{H}, 1.7873100476,0.800228881,1$ $.4877970476 \backslash \mathrm{C},-0.6218359524,3.628615881,-0.2715009524 \backslash \mathrm{C},-0.8346519524$, $2.370814881,0.5425200476 \backslash \mathrm{H},-0.3375089524,3.388835881,-1.3024259524 \backslash \mathrm{H},-$ $1.0274519524,2.542870881,1.6049850476 \backslash \mathrm{H},-1.5287339524,1.645684881,0.11$ $09700476 \backslash \mathrm{H}, 0.1662790476,4.265612881,0.1502810476 \backslash \mathrm{H},-1.5351179524,4.240$ $871881,-0.3276109524 \backslash \backslash$ Version=Al64T-G03RevC.02 $\backslash$ State $=2-A \backslash H F=-599.10469$ $33 \backslash S 2=0.757023 \backslash S 2-1=0 . \backslash S 2 A=0.750026 \backslash R M S D=5.645 e-09 \backslash R M S F=3.735 e-06 \backslash D i p o$ $l e=0.7198576,1.1468175,0.5246881 \backslash \mathrm{PG}=\mathrm{C} 01[\mathrm{X}(\mathrm{C} 5 \mathrm{H} 13 \mathrm{~F} 104)] \backslash \backslash \mathrm{a}$

\section{FH $\cdots 4 a \cdot \cdots \mathrm{OCHO}^{-}$}

$1 \backslash 1 \backslash$ GINC-SC102\FOpt \RB3LYP \6-31G(d,p) \C3H8F104 (1-) \GMS501\06-Jun-2005 $0 \backslash \backslash \#$ B3LYP/6-31G** OPT FREQ=NORAMAN \\HF gemdiol ethylene glycol radica

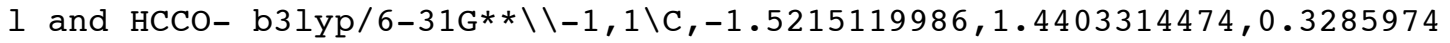
$938 \backslash C,-0.510918425,0.3468206548,0.011620191 \backslash 0,0.2044055462,0.647708049$ $5,-1.1175949152 \backslash \mathrm{H}, 0.1477645641,0.1262792055,0.8604816279 \backslash 0,-1.27245847$ $15,-0.9113724926,-0.2373326502 \backslash \mathrm{H},-1.0809005158,-1.0636263891,-1.174573$ $025 \backslash \mathrm{H}, 1.1642835413,0.2706612344,-1.0105239173 \backslash \mathrm{O}, 2.5630106995,-0.294445$ $3557,-0.8939063095 \backslash \mathrm{C}, 3.0152715991,-0.341875866,0.2955775877 \backslash 0,2.452161$ $4059,-0.0687705559,1.3694296275 \backslash \mathrm{H}, 4.0865293926,-0.6874407929,0.3514549$ $345 \backslash \mathrm{F},-3.7412586318,-0.7332083426,0.3233011122 \backslash \mathrm{H},-2.8101789598,-0.8436$ $920144,0.0979122307 \backslash \mathrm{H},-2.2285755103,1.1166470347,1.0962198574 \backslash \mathrm{H},-0.995$ $0263798,2.3381188054,0.6668301804 \backslash \mathrm{H},-2.0865689405,1.68531342,-0.577049$ $5544 \backslash \backslash$ Version=Al64T-G03RevC . 02 \State $=1-A \backslash H F=-519.960036 \backslash$ RMSD $=2.942 e-09$ $\backslash \mathrm{RMSF}=2.666 \mathrm{e}-05 \backslash \mathrm{Dipole}=-1.0841014,0.211034,-0.4435408 \backslash \mathrm{PG}=\mathrm{C} 01 \quad[\mathrm{X}(\mathrm{C} 3 \mathrm{H} 8 \mathrm{~F} 1$ 04 ) $] \backslash \backslash$ @

\section{$\mathrm{NH}_{4}^{+} \cdots \cdot 1 \mathrm{a} \cdots \cdot \mathrm{NH}_{3}$}

$1 \backslash 1 \backslash$ GINC-RADOM-BAROSSA036 $\backslash$ FOpt $\backslash R B 3 L Y P \backslash 6-31 G(d, p) \backslash C 2 H 13 N 2 O 2(1+) \backslash$ SANDAL

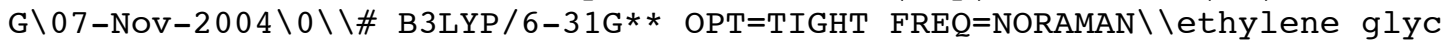
ol, H-bond from $\mathrm{NH} 4+$ and donating to $\mathrm{nh} 3 \backslash \backslash 1,1 \backslash \mathrm{C}, 0.7407521505,1.246718$ $9302,-0.0130684924 \backslash 0,1.1820483906,-0.0643482212,-0.4612453148 \backslash \mathrm{H}, 0.4072$ $728845,-0.6212169507,-0.1567104722 \backslash \mathrm{H}, 1.1186234622,1.9995349201,-0.7071$ $890263 \backslash \mathrm{H}, 1.1330081013,1.4362893704,0.9928437905 \backslash \mathrm{C},-0.7897603361,1.1756$ $987949,0.0044998656 \backslash \mathrm{O},-1.0811889338,-0.1100847002,0.5260908086 \backslash \mathrm{H},-2.01$ $12528004,-0.3931008142,0.2586412471 \backslash \mathrm{H}, 2.4046502341,-0.495971797,-0.122$ $9436089 \backslash \mathrm{N}, 3.4970526219,-0.8909093641,0.0934968607 \backslash \mathrm{H}, 3.6571171902,-1.03$ $20136872,1.0924212691 \backslash \mathrm{H}, 3.6482252137,-1.781835796,-0.3835755207 \backslash \mathrm{H}, 4.19$ $2667297,-0.2271840391,-0.2522892511 \backslash \mathrm{N},-3.6075067831,-0.871239747,-0.12$ $71899036 \backslash \mathrm{H},-4.2938062588,-0.1628066732,0.1262549012 \backslash \mathrm{H},-3.7787237917,-1$ $.1036980442,-1.1035503106 \backslash \mathrm{H},-3.8440125929,-1.7018115127,0.4128740035 \backslash \mathrm{H}$ $,-1.1918332685,1.9853000762,0.6269377051 \backslash \mathrm{H},-1.1815830831,1.2945157467$, $-1.0152156164 \backslash \backslash$ Version=x86-Linux-G03RevB.02 $\backslash$ State $=1-A \backslash H F=-343.7992659 \backslash$ $\mathrm{RMSD}=8.293 e-09 \backslash \mathrm{RMSF}=2.026 \mathrm{e}-06 \backslash \mathrm{Dipole}=2.2629537,-1.0179875,-0.0865882 \backslash \mathrm{P}$ $\mathrm{G}=\mathrm{C} 01[\mathrm{X}(\mathrm{C} 2 \mathrm{H} 13 \mathrm{~N} 2 \mathrm{O} 2)] \backslash \backslash @$ 


\section{TS: $\mathrm{NH}_{4}{ }^{+} \cdots 1 \mathrm{a} \cdots \cdot \mathrm{NH}_{3} \rightarrow \mathrm{NH}_{4}{ }^{+} \cdots 2 \mathrm{a} \cdots \cdot \mathrm{NH}_{3}$}

$1 \backslash 1 \backslash$ GINC-RADOM-BAROSSA086 \FTS \UB3LYP \6-31G $(\mathrm{d}, \mathrm{p}) \backslash \mathrm{C} 4 \mathrm{H} 18 \mathrm{~N} 2 \mathrm{O} 3(1+, 2) \backslash$ SANDA

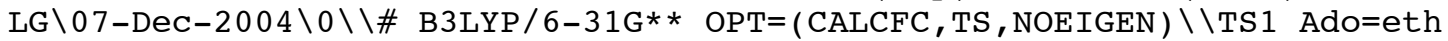
anol ethylene glycol, H-bond from $\mathrm{NH} 4+$ and donating to $\mathrm{nh} 3 \backslash \backslash 1,2 \backslash \mathrm{C}, 1.2$ $086057365,-0.8394390067,0.4677386672 \backslash 0,2.5339439318,-0.2103583701,0.45$ $66753699 \backslash \mathrm{H}, 2.3242424364,0.663628515,0.0388514645 \backslash \mathrm{H}, 1.1438077129,-1.479$ $5756356,1.3489773854 \backslash \mathrm{H}, 1.1065186478,-1.4450252997,-0.4411969323 \backslash \mathrm{C}, 0.19$ $05963763,0.2837008566,0.4861663765 \backslash 0,0.6736316407,1.2682811068,-0.3747$ $182127 \backslash \mathrm{H}, 0.2073695345,2.1501077699,-0.2094241822 \backslash \mathrm{H}, 3.5759741386,-0.882$ $5463831,-0.0700572259 \backslash \mathrm{N}, 4.5262559028,-1.4620417816,-0.4555057153 \backslash \mathrm{H}, 4.4$ $499514715,-1.6951904725,-1.4471417966 \backslash \mathrm{H}, 5.363320002,-0.890765323,-0.32$ $41752945 \backslash \mathrm{H}, 4.6516218253,-2.3295470373,0.0697394999 \backslash \mathrm{N},-0.5985232678,3.6$ $277017694,0.0026886638 \backslash \mathrm{H},-1.6049988662,3.5320616088,-0.1193839525 \backslash \mathrm{H},-0$ $.4568822569,4.0807741608,0.9034160753 \backslash \mathrm{H},-0.2868894997,4.2895659247,-0$. $7059851773 \backslash \mathrm{H},-0.933038648,-0.2093447734,0.0245052317 \backslash \mathrm{H},-0.0344807148,0$ $.6526228465,1.4952282438 \backslash \mathrm{C},-3.131607619,-1.034139229,0.4010363496 \backslash \mathrm{C},-2$ $.1071432652,-0.6557551055,-0.6372672907 \backslash \mathrm{H},-3.338996741,-0.172770729,1$. $0572044414 \backslash \mathrm{O},-4.3061532475,-1.4489797848,-0.2906272963 \backslash \mathrm{H},-1.7578225416$ $,-1.4877127575,-1.251732841 \backslash \mathrm{H},-2.3687957914,0.2151759366,-1.2403297596$ $\backslash \mathrm{H},-2.7397327345,-1.8423462105,1.0402116984 \backslash \mathrm{H},-4.9693823908,-1.7064807$ $629,0.3643289806 \backslash \backslash$ Version $=x 86-L$ inux-G03RevB. $02 \backslash$ State $=2-A \backslash H F=-498.15295$ $57 \backslash \mathrm{S} 2=0.756689 \backslash \mathrm{S} 2-1=0 . \backslash \mathrm{S} 2 \mathrm{~A}=0.750023 \backslash \mathrm{RMSD}=8.451 \mathrm{e}-09 \backslash \mathrm{RMSF}=1.089 \mathrm{e}-06 \backslash \mathrm{Dipo}$ $l e=4.9670969,-0.0573285,0.2335397 \backslash P G=C 01 \quad[X(C 4 \mathrm{H} 18 \mathrm{~N} 2 \mathrm{O} 3)] \backslash \backslash @$

\section{$\mathrm{NH}_{4}{ }^{+} \cdots 2 \mathrm{a} \cdots \cdot \mathrm{NH}_{3}$}

$1 \backslash 1 \backslash$ GINC-RADOM-BAROSSA053\FOpt \UB3LYP \6-31G(d,p) \C2H12N2O2 $(1+, 2) \backslash$ SAND ALG $\backslash 07-N O v-2004 \backslash 0 \backslash \backslash \#$ B3LYP/6-31G** OPT=TIGHT FREQ=NORAMAN \ethylene gl ycol radical, H-bond from $\mathrm{NH} 4+$ and donating to $\mathrm{nh} 3 \backslash \backslash 1,2 \backslash \mathrm{C}, 0.6125551282$ $, 0.93508996,0.1729671437 \backslash 0,1.4551154258,-0.1965694901,-0.4673142634 \backslash \mathrm{H}$, $0.9132834896,-0.9974381919,-0.3767870836 \backslash \mathrm{H}, 1.026948928,1.8455512034,-0$ $.2574690556 \backslash \mathrm{H}, 0.8235676255,0.8684790887,1.24511209 \backslash \mathrm{C},-0.8008942032,0.7$ $655779132,-0.1184946592 \backslash \mathrm{O},-1.4038987957,-0.2614689028,0.4964679666 \backslash \mathrm{H},-$ $1.2935863281,1.2725444973,-0.943045886 \backslash \mathrm{H},-2.3869581004,-0.3221529582,0$ $.2339134625 \backslash \mathrm{H}, 2.738690807,-0.3440875066,-0.1262464029 \backslash \mathrm{N}, 3.905061564,-0$ $.4438871682,0.081880842 \backslash \mathrm{H}, 4.1171257989,-0.4058483661,1.0804148076 \backslash \mathrm{H}, 4$. $2619946287,-1.3276234111,-0.2863630736 \backslash \mathrm{H}, 4.408645497,0.3149564978,-0.3$ $815937632 \backslash \mathrm{N},-4.0085469696,-0.4232264793,-0.1151488499 \backslash \mathrm{H},-4.5146636587$, $0.4242113854,0.1353377552 \backslash \mathrm{H},-4.2341027569,-0.6227754842,-1.0877613509 \backslash$ $\mathrm{H},-4.4162466823,-1.1757213174,0.4373000225 \backslash \backslash$ Version=x86-Linux-G03RevB. $02 \backslash \mathrm{State}=2-\mathrm{A} \backslash \mathrm{HF}=-343.1405806 \backslash \mathrm{S} 2=0.75323 \backslash \mathrm{S} 2-1=0 . \backslash \mathrm{S} 2 \mathrm{~A}=0.750008 \backslash \mathrm{RMSD}=8.00$ $3 e-09 \backslash \mathrm{RMSF}=1.159 \mathrm{e}-06 \backslash \mathrm{Dipole}=2.494799,-0.6097384,-0.1280836 \backslash \mathrm{PG}=\mathrm{C} 01 \quad[\mathrm{X}(\mathrm{C}$ $2 \mathrm{H} 12 \mathrm{~N} 2 \mathrm{O} 2)] \backslash \backslash @$

\section{$\mathrm{NH}_{4}{ }^{+} \cdots 3 \mathrm{a} \cdots \cdot \mathrm{NH}_{3}$}

$1 \backslash 1 \backslash$ GINC-RADOM-BAROSSA116 $\backslash$ Freq \UB3LYP \6-31G (d,p) \C2H12N2O2 $(1+, 2) \backslash$ SAND ALG $\backslash 12-N O v-2004 \backslash 0 \backslash \backslash \#$ B3LYP/6-31G** FREQ=NORAMAN \gemdiolethane radical

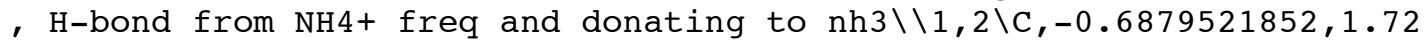
$45038889,0.1956179074 \backslash \mathrm{H},-1.2672831852,2.0390458889,1.0565359074 \backslash \mathrm{H},-0.6$ $548391852,2.3493338889,-0.6895060926 \backslash \mathrm{C}, 0.1487058148,0.5045538889,0.240$ $2989074 \backslash \mathrm{O}, 1.1967668148,0.5797488889,-0.6094720926 \backslash \mathrm{H}, 0.4043988148,0.193$ $8618889,1.2601439074 \backslash \mathrm{O},-0.7182561852,-0.7061731111,-0.2324780926 \backslash \mathrm{H},-0$. $4414051852,-0.8037361111,-1.1601400926 \backslash \mathrm{H}, 1.9893128148,0.0406968889,-0$. $2699500926 \backslash \mathrm{H},-2.0771481852,-0.7073381111,-0.0663720926 \backslash \mathrm{N},-3.2326831852$ $,-0.7682381111,0.1078659074 \backslash \mathrm{H},-3.6790311852,-1.3566161111,-0.598379092$ $6 \backslash \mathrm{H},-3.4329991852,-1.1681261111,1.0266559074 \backslash \mathrm{H},-3.6498671852,0.1631698$ $889,0.0614699074 \backslash \mathrm{N}, 3.3815998148,-0.7271811111,0.2469899074 \backslash \mathrm{H}, 3.8499768$ $148,-0.2159051111,0.9929929074 \backslash \mathrm{H}, 4.0288718148,-0.7381391111,-0.5398810$ $926 \backslash \mathrm{H}, 3.2949898148,-1.6912661111,0.5625399074 \backslash \backslash$ Version=x86-Linux-G03Re 
VB. 02 \State $=2-A \backslash H F=-343.1334232 \backslash \mathrm{S} 2=0.753713 \backslash \mathrm{S} 2-1=0 . \backslash \mathrm{S} 2 \mathrm{~A}=0.750009 \backslash \mathrm{RMSD}=$ $4.339 \mathrm{e}-09 \backslash \mathrm{RMSF}=6.898 \mathrm{e}-06 \backslash \mathrm{Dipole}=-2.2675095,-1.3254798,0.4687197 \backslash \mathrm{PG}=\mathrm{C} 0$

$1[\mathrm{X}(\mathrm{C} 2 \mathrm{H} 12 \mathrm{~N} 2 \mathrm{O} 2)] \backslash \backslash @$

\section{TS: $\mathrm{NH}_{4}{ }^{+} \cdots 3 \mathrm{a} \cdots \cdots \mathrm{NH}_{3} \rightarrow \mathrm{NH}_{4}{ }^{+} \cdots 4 \mathrm{a} \cdots \cdot \mathrm{NH}_{3}$}

$1 \backslash 1 \backslash$ GINC-RADOM-BAROSSA0 74 $\backslash$ Freq $\backslash U B 3 L Y P \backslash 6-31 G(d, p) \backslash C 4 H 18 N 2 O 3(1+, 2) \backslash$ SAND

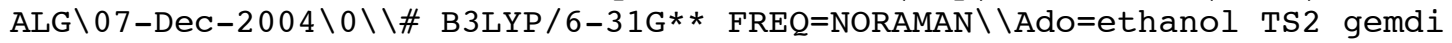

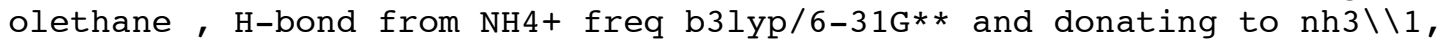
$2 \backslash \mathrm{C}, 0.1201901,-0.9844611625,-0.1563570375 \backslash \mathrm{C}, 1.0383931,0.1686148375,0.0$ $881319625 \backslash 0,0.7954311,1.1959978375,-0.7548760375 \backslash \mathrm{H}, 1.0987301,0.4518618$ $375,1.1457199625 \backslash 0,2.5131571,-0.2772921625,-0.1822300375 \backslash \mathrm{H}, 2.6651821,0$ $.0325808375,-1.0915600375 \backslash \mathrm{H}, 1.0572591,2.0836108375,-0.3410360375 \backslash \mathrm{H}, 2.9$ $164541,-1.5149171625,0.0386609625 \backslash \mathrm{N}, 3.3457501,-2.6094151625,0.28400496$ $25 \backslash \mathrm{H}, 4.2499781,-2.7565281625,-0.1680540375 \backslash \mathrm{H}, 3.4770071,-2.7226861625,1$ $.2907279625 \backslash \mathrm{H}, 2.7055291,-3.3365721625,-0.0386120375 \backslash \mathrm{N}, 1.3557891,3.6187$ $978375,0.2899489625 \backslash \mathrm{H}, 0.6774451,3.8903298375,0.9993779625 \backslash \mathrm{H}, 1.2467941$, $4.2822308375,-0.4756560375 \backslash \mathrm{H}, 2.2775681,3.7874548375,0.6878689625 \backslash \mathrm{H}, 0.2$ $533211,-1.8353071625,0.5138829625 \backslash \mathrm{H},-1.1195019,-0.5217111625,0.1396469$ $625 \backslash \mathrm{H}, 0.0421581,-1.2638261625,-1.2078380375 \backslash \mathrm{C},-3.3849469,-0.8702331625$ $,-0.2036750375 \backslash \mathrm{C},-2.3256709,0.0047618375,0.4228259625 \backslash \mathrm{H},-3.3057219,-1$. $8969041625,0.1887189625 \backslash \mathrm{O},-4.6414849,-0.2867611625,0.1206819625 \backslash \mathrm{H},-2.2$ $516589,1.0024968375,-0.0118040375 \backslash \mathrm{H},-2.3543289,0.0261078375,1.51404996$ $25 \backslash \mathrm{H},-3.2327679,-0.9203081625,-1.2933340375 \backslash \mathrm{H},-5.3388399,-0.8212421625$ ,$-0.2826000375 \backslash \backslash$ Version=x86-Linux-G03RevB.02 $\backslash$ State $=2-A \backslash H F=-498.158356 \backslash$ $\mathrm{S} 2=0.756695 \backslash \mathrm{S} 2-1=0 . \backslash \mathrm{S} 2 \mathrm{~A}=0.750024 \backslash \mathrm{RMSD}=5.433 \mathrm{e}-09 \backslash \mathrm{RMSF}=1.071 \mathrm{e}-06 \backslash \mathrm{Dipole}=$ $4.5255556,-1.6919601,0.349706 \backslash \mathrm{PG}=\mathrm{C} 01 \quad[\mathrm{X}(\mathrm{C} 4 \mathrm{H} 18 \mathrm{~N} 2 \mathrm{O} 3)] \backslash \backslash \mathrm{a}$

\section{$\mathrm{NH}_{4}^{+} \cdots 4 \mathrm{a} \cdots \mathrm{NH}_{3}$}

$1 \backslash 1 \backslash$ GINC-RADOM-BAROSSA023\FOpt \RB3LYP \6-31G(d,p) \C2H13N2O2(1+) \SANDAL

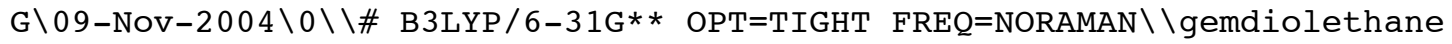
, H-bond from $\mathrm{NH} 4+$ and donating to $\mathrm{nh} 3 \backslash \backslash 1,1 \backslash \mathrm{C},-0.6537450473,1.7576639$ $824,0.18323 \backslash \mathrm{C}, 0.1579189871,0.4784910043,0.22061 \backslash 0,1.2172869854,0.54158$ $50328,-0.623518 \backslash \mathrm{H}, 0.4221809951,0.1809610114,1.243355 \backslash 0,-0.7199309819,-$ $0.6710370194,-0.248988 \backslash \mathrm{H},-0.4059709778,-0.8254530109,-1.155863 \backslash \mathrm{H}, 1.995$ $1710002,-0.0064549463,-0.279861 \backslash \mathrm{H},-2.0997609802,-0.7349040566,-0.06196$ $\backslash \mathrm{N},-3.2301159767,-0.865732087,0.126351 \backslash \mathrm{H},-3.5781549543,-1.6981800964,-$ $0.354009 \backslash \mathrm{H},-3.4106469737,-0.9773870919,1.126204 \backslash \mathrm{H},-3.7554389985,-0.055$ $9421012,-0.208859 \backslash \mathrm{N}, 3.3941380215,-0.7997779086,0.255969 \backslash \mathrm{H}, 3.8229700092$ $,-0.341510897,1.057963 \backslash \mathrm{H}, 4.0724960199,-0.7380358903,-0.501782 \backslash \mathrm{H}, 3.3158$ $160481,-1.7870049107,0.491918 \backslash \mathrm{H},-1.5559510454,1.6849189581,0.799747 \backslash \mathrm{H}$, $-0.0476460695,2.5792189987,0.572339 \backslash \mathrm{H},-0.9271110538,1.997027975,-0.848$ $429 \backslash \backslash$ Version=x86-Linux-G03RevB.02 $\backslash$ State $=1-A \backslash H F=-343.8088183 \backslash \mathrm{RMSD}=5.597$ e-09 $\backslash$ RMSF $=8.038 e-07 \backslash D i p o l e=-2.3981088,-1.5757646,0.501509 \backslash P G=C 01]$ [X(C2 $\mathrm{H} 13 \mathrm{~N} 2 \mathrm{O} 2$ ) ] \\@

\section{$7 a$}

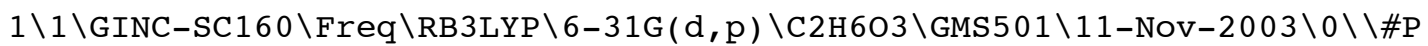
B3LYP/6-31G(D,P) FREQ TEST MAXDISK=19660800 GFINPUT IOP $(6 / 7=3) \backslash$ Confor mer \#4 h2oglycol freq (-305.489199374)b3lyp/6-31G(d,p)usegeom oflo west tight calc $\backslash \backslash 0,1 \backslash 0,-1.563434881,0.0326620238,-0.5694439286 \backslash \mathrm{C},-0.91$ $5216881,-0.0543889762,0.6959340714 \backslash \mathrm{C}, 0.593684119,0.0318590238,0.480323$ $0714 \backslash \mathrm{H},-1.255607881,0.7985520238,1.2883340714 \backslash \mathrm{H},-1.171043881,-0.981442$ $9762,1.2262930714 \backslash 0,0.932453119,1.1911800238,-0.2292959286 \backslash 0,0.9306351$ $19,-1.1422499762,-0.2517179286 \backslash \mathrm{H}, 1.144797119,0.0739270238,1.4292390714$ $\backslash \mathrm{H},-1.222993881,-0.7169299762,-1.0818649286 \backslash \mathrm{H}, 0.227165119,1.2962530238$ $,-0.8913609286 \backslash \mathrm{H}, 1.809653119,-0.9879159762,-0.6245209286 \backslash \backslash$ Version=DECAXP-OSF / 1-G03RevB.03 \State $=1-\mathrm{A} \backslash \mathrm{HF}=-305.4891994 \backslash \mathrm{RMSD}=4.450 \mathrm{e}-09 \backslash \mathrm{RMSF}=5.1$ $20 e-06 \backslash$ Dipole $=0.248907,-0.3204469,0.0219802 \backslash \mathrm{PG}=\mathrm{C} 01[\mathrm{X}(\mathrm{C} 2 \mathrm{H} 6 \mathrm{O} 3)] \backslash \backslash @$ 


\section{TS:7a $\rightarrow \mathbf{8 a}$}

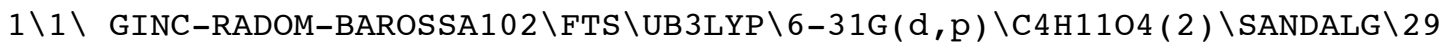

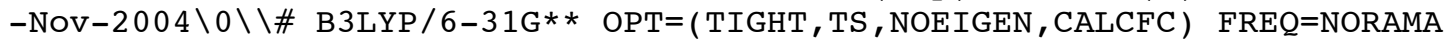

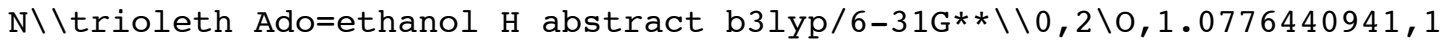
$.130099563,1.5897719622 \backslash \mathrm{C}, 0.5217530231,0.9597647608,0.3164615272 \backslash \mathrm{C},-0$. $9182972863,1.4376066681,0.2836063761 \backslash \mathrm{H}, 1.1513930463,1.4634198031,-0.42$ $01901552 \backslash \mathrm{H}, 0.5316599572,-0.3135599193,-0.0108130422 \backslash 0,-1.0211377607,2$. $8198303346,0.5361967934 \backslash 0,-1.5833199338,0.6846078677,1.2907448802 \backslash \mathrm{H},-1$ $.3776509476,1.2788914299,-0.7002237411 \backslash \mathrm{H}, 0.4363618234,0.737117553,2.20$ $48261087 \backslash \mathrm{H},-0.4031424362,3.0038688574,1.2619301782 \backslash \mathrm{H},-2.428448728,1.12$ $58083318,1.4535767943 \backslash \mathrm{C}, 0.6973117459,-1.9176536329,-1.7367033944 \backslash \mathrm{C}, 0.5$ $614880923,-1.706909817,-0.2541592578 \backslash \mathrm{H},-0.1470656393,-1.4423528797,-2$. $2630348999 \backslash 0,0.7241153468,-3.3258408868,-1.9957582788 \backslash \mathrm{H}, 1.6200600096,-$ $1.4373878225,-2.1009382793 \backslash \mathrm{H}, 1.4225633692,-2.0320932317,0.3315964146 \backslash \mathrm{H}$ $,-0.3852644075,-2.0432979845,0.1699282914 \backslash \mathrm{H}, 0.8275865325,-3.4468370392$ ,$-2.9495320326 \backslash \backslash$ Version=x86-Linux-G03RevB.02 \State=2-A \HF=-459.8422202 $\backslash \mathrm{S} 2=0.756791 \backslash \mathrm{S} 2-1=0 . \backslash \mathrm{S} 2 \mathrm{~A}=0.750023 \backslash \mathrm{RMSD}=3.589 \mathrm{e}-09 \backslash \mathrm{RMSF}=1.402 \mathrm{e}-06 \backslash \mathrm{Dipole}$ $=-0.3949446,0.4279518,-0.2387939 \backslash \mathrm{PG}=\mathrm{C} 01[\mathrm{X}(\mathrm{C} 4 \mathrm{H} 1104)] \backslash \backslash @$

\section{$8 a$}

$1 \backslash 1 \backslash G I N C-S C 10 \backslash F O p t \backslash U B 3 L Y P \backslash 6-31 G(d, p) \backslash C 2 H 5 O 3(2) \backslash G M S 501 \backslash 21-N o v-2003 \backslash 0 \backslash \backslash \#$ P B3LYP/6-31G(D,P) OPT=(TIGHT,READFC) FREQ TEST MAXDISK=26214400 GUESS

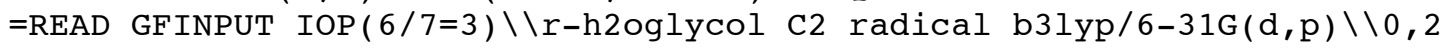
$\backslash 0,1.905934514,-0.0428860719,-0.1074025177 \backslash \mathrm{C}, 0.7462336454,-0.747835422$ $9,-0.0452149227 \backslash \mathrm{C},-0.4999691222,-0.0323963733,0.3739007568 \backslash \mathrm{H}, 0.8735157$ $826,-1.7996306416,0.1864192474 \backslash 0,-1.6563942318,-0.6746191513,-0.096428$ $9849 \backslash 0,-0.3517503283,1.2947638296,-0.1282179957 \backslash \mathrm{H},-0.6379684895,0.0046$ $191059,1.4679206081 \backslash \mathrm{H}, 1.642944557,0.8894715866,-0.2044593233 \backslash \mathrm{H},-1.4954$ $457784,-0.8731502391,-1.0321671523 \backslash \mathrm{H},-1.0429528423,1.8420121129,0.2665$ $676019 \backslash \backslash$ Version=DEC-AXP-OSF $/ 1-$ G03RevB.03 \State $=2-A \backslash H F=-304.8278092 \backslash \mathrm{S} 2=$ $0.752983 \backslash \mathrm{S} 2-1=0 . \backslash \mathrm{S} 2 \mathrm{~A}=0.750006 \backslash \mathrm{RMSD}=6.308 \mathrm{e}-09 \backslash \mathrm{RMSF}=3.246 \mathrm{e}-06 \backslash \mathrm{Dipole}=-0$. $3366524,0.4514264,0.1186608 \backslash P G=C 01 \quad[\mathrm{X}(\mathrm{C} 2 \mathrm{H} 503)] \backslash \backslash @$

\section{9a}

$1 \backslash 1 \backslash G I N C-L C 87 \backslash F O p t \backslash U B 3 L Y P \backslash 6-31 G(d, p) \backslash C 2 H 3 O 2(2) \backslash G M S 501 \backslash 30-S e p-2003 \backslash 0 \backslash \backslash \#$ B3LYP/6-31G(D,P) OPT=(CALCFC, TIGHT) MAXDISK=26214400 FREQ TEST\\glyCol $\mathrm{rad} \mathrm{b} 3 \mathrm{lyp}_{\mathrm{y}} / 6-31 \mathrm{G}(\mathrm{d}, \mathrm{p}) \backslash \backslash 0,2 \backslash \mathrm{H}, 1.3073985455,-0.3853154949,0 . \backslash 0,1.29593773$ $89,0.6006251825,0 . \backslash \mathrm{C}, 0.0003829831,0.9021486442,0 . \backslash \mathrm{C},-0.9239499425,-0.1$ $76386933,0 . \backslash \mathrm{O},-0.4863323374,-1.3476252198,0 . \backslash \mathrm{H},-0.241556123,1.95801651$ $42,0 . \backslash \mathrm{H},-2.001283878,0.0487290114,0 . \backslash \backslash$ Version=x86-Linux-G03RevB.03 \Sta te $=2-\mathrm{A} " \backslash \mathrm{HF}=-228.4168067 \backslash \mathrm{S} 2=0.759149 \backslash \mathrm{S} 2-1=0 . \backslash \mathrm{S} 2 \mathrm{~A}=0.75003 \backslash \mathrm{RMSD}=3.931 \mathrm{e}-09$ $\backslash \mathrm{RMSF}=4.729 \mathrm{e}-07 \backslash \mathrm{Dipole}=-0.2019813,0.7824275,0 . \backslash \mathrm{PG}=\mathrm{CS} \quad[\mathrm{SG}(\mathrm{C} 2 \mathrm{H} 3 \mathrm{O} 2)] \backslash \backslash @$

\section{0a}

$1 \backslash 1 \backslash G I N C-S C 7 \backslash F T S \backslash U B 3 L Y P \backslash 6-31 G(d, p) \backslash C 2 H 3 O 2(2) \backslash G M S 501 \backslash 02-D e C-2003 \backslash 0 \backslash \backslash \# B 3$ LYP / 6-31G (D, P ) OPT=( TS , NOEIGEN , CALCFC , TIGHT , MAXCYCLE=200) MAXDISK=1966


, 0 , $1.167815599,0.5851021147 \backslash 0,0 \ldots,-1.167815599,0.5851021147 \backslash \mathrm{C}, 0 ., 0.712$ $1798276,-0.6197418915 \backslash \mathrm{C}, 0 .,-0.7121798276,-0.6197418915 \backslash \mathrm{H}, 0 ., 1.37396066$ $57,-1.4809357454 \backslash \mathrm{H}, 0 .,-1.3739606657,-1.4809357454 \backslash \backslash$ Version=DEC-AXP-OSF $/ 1-G 03 R e v B .03 \backslash$ State $=2-B 1 \backslash H F=-228.4032095 \backslash \mathrm{S} 2=0.753382 \backslash \mathrm{S} 2-1=0 . \backslash \mathrm{S} 2 \mathrm{~A}=0.750$ $007 \backslash \mathrm{RMSD}=5.465 \mathrm{e}-09 \backslash \mathrm{RMSF}=1.716 \mathrm{e}-08 \backslash \mathrm{Dipole}=0,0 .,-0.993754 \backslash \mathrm{PG}=\mathrm{C} 02 \mathrm{~V}[\mathrm{C} 2(\mathrm{H}$ 1 ) , $\mathrm{SGV}(\mathrm{C} 2 \mathrm{H} 2 \mathrm{O} 2)] \backslash \backslash \mathrm{Q}$ 


\section{TS:9a $\rightarrow 11 a$}

$1 \backslash 1 \backslash$ GINC-RADOM-BAROSSA $140 \backslash F T S \backslash U B 3 L Y P \backslash 6-31 G(d, p) \backslash C 4$ H9O3 (2) \SANDALG \29-

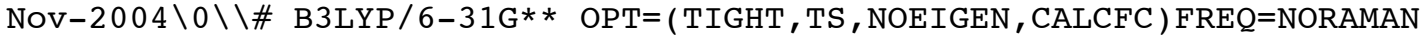


$.7367955512,-1.5359543933 \backslash 0,1.0950899528,-0.1859749474,-1.3890489649 \backslash \mathrm{C}$ $,-0.2898569759,-0.1358886123,-1.3810224722 \backslash \mathrm{H},-0.7373795609,-1.01566009$ $13,-1.8576004799 \backslash \mathrm{H},-0.6973804751,-0.2230706595,-0.213474219 \backslash \mathrm{C},-0.82434$ $77306,1.1558205272,-1.8850728015 \backslash 0,-0.0842276095,2.1102035186,-2.07113$ $74723 \backslash \mathrm{H},-1.919981653,1.2285318499,-2.0268550488 \backslash \mathrm{C}, 0.4121825331,-1.0381$ $328219,1.7477056252 \backslash \mathrm{C},-0.8984607668,-0.4498821406,1.31419945 \backslash \mathrm{H}, 1.23688$ $33947,-0.407409732,1.3844024042 \backslash \mathrm{H}, 0.5452238713,-2.0337886515,1.2976478$ $651 \backslash 0,0.4110713386,-1.114884419,3.1783434658 \backslash \mathrm{H},-1.7694216847,-1.103717$ $7175,1.3301943883 \backslash \mathrm{H},-1.096226366,0.568049164,1.6487918557 \backslash \mathrm{H}, 1.28792613$ $98,-1.4159846445,3.4527325899 \backslash \backslash$ Version=x86-Linux-G03RevB .02 S State=2-A \} $\mathrm{HF}=-383.4089358 \backslash \mathrm{S} 2=0.757852 \backslash \mathrm{S} 2-1=0 . \backslash \mathrm{S} 2 \mathrm{~A}=0.750037 \backslash \mathrm{RMSD}=5.273 e-09 \backslash \mathrm{RMSF}=1$ $.373 e-06 \backslash$ Dipole $=-0.2383445,-0.531391,-0.0501214 \backslash \mathrm{PG}=\mathrm{C} 01 \quad[\mathrm{X}(\mathrm{C} 4 \mathrm{H} 903)] \backslash \backslash \mathrm{Q}$

\section{$11 \mathrm{a}$}

$1 \backslash 1 \backslash G I N C-L C 72 \backslash F O p t \backslash R B 3 L Y P \backslash 6-31 G(d, p) \backslash C 2 H 402 \backslash G M S 501 \backslash 30-$ Sep-2003 $\backslash 0 \backslash \backslash \# B 3 L$ YP $/ 6-31 \mathrm{G}(\mathrm{D}, \mathrm{P})$ OPT $=($ CALCFC $, \mathrm{TIGHT}, \mathrm{MAXCYCLE}=100) \mathrm{MAXDISK}=26214400 \mathrm{FREQTE}$ $\mathrm{ST} \backslash \backslash \mathrm{Glycolaldehyde} \mathrm{b} 3 \mathrm{lyp} / 6-31 \mathrm{G}(\mathrm{d}, \mathrm{p}) \backslash \backslash 0,1 \backslash \mathrm{H}, 1.3332547117,-0.4245004778$, $0 . \backslash 0,1.3442501569,0.5489306851,0 . \backslash \mathrm{C}, 0.0011489655,0.9358595101,0 . \backslash \mathrm{H},-0$. $2466929443,1.5539256172,0.8812125016 \backslash \mathrm{H},-0.2466929443,1.5539256172,-0.8$ $812125016 \backslash \mathrm{C},-0.9286323462,-0.2541516044,0 . \backslash 0,-0.5015622347,-1.39074570$ $23,0 . \backslash \mathrm{H},-2.0164719162,-0.0390780533,0 . \backslash \backslash$ Version=x86-Linux-G03RevB.03\S tate $=1-\mathrm{A}^{\prime} \backslash \mathrm{HF}=-229.0456028 \backslash \mathrm{RMSD}=7.255 \mathrm{e}-09 \backslash \mathrm{RMSF}=1.179 \mathrm{e}-06 \backslash \mathrm{Dipole}=-0.7666$ $453,0.4912846,0 . \backslash P G=C S[S G(\mathrm{C} 2 \mathrm{H} 2 \mathrm{O} 2), \mathrm{X}(\mathrm{H} 2)] \backslash \backslash @$

\section{$\mathrm{CN}^{-} \cdot . \cdot 9 \mathrm{a}$}

$1 \backslash 1 \backslash G I N C-S C 122 \backslash F O p t \backslash U B 3 L Y P \backslash 6-31 G(d, p) \backslash C 3 H 3 N 1 O 2(1-, 2) \backslash G M S 501 \backslash 31-M a r-200$ $5 \backslash 0 \backslash \backslash \#$ B3LYP/6-31G** OPT FREQ=NORAMAN \\follow imag freq cyanide a

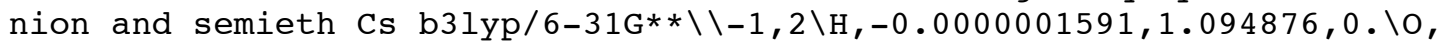
$1.4780899679,0.2210622148,0 . \backslash 0,-0.9886687968,-1.3986461437,0 . \backslash \mathrm{C}, 1.3902$ $901526,-1.050525798,0 . \backslash \mathrm{C}, 0.1935222658,-1.8294279719,0 . \backslash \mathrm{H}, 2.3273072402$, $-1.6527996618,0 . \backslash \mathrm{H}, 0.3927174262,-2.9329599429,0 . \backslash \mathrm{C},-0.8068672721,1.872$ $7698828,0 . \backslash \mathrm{N},-1.6138653935,2.7078087655,0 . \backslash \backslash$ Version=A164T-G03RevC.02\S tate $=2-\mathrm{A}^{\prime} \backslash \mathrm{HF}=-321.3089488 \backslash \mathrm{S} 2=0.756527 \backslash \mathrm{S} 2-1=0 . \backslash \mathrm{S} 2 \mathrm{~A}=0.750017 \backslash \mathrm{RMSD}=6.261 \mathrm{e}$ $-09 \backslash \mathrm{RMSF}=1.898 \mathrm{e}-05 \backslash \mathrm{Dipole}=0.8201583,-0.6382763,0 . \backslash \mathrm{PG}=\mathrm{CS} \quad[\mathrm{SG}(\mathrm{C} 3 \mathrm{H} 3 \mathrm{~N} 102)]$ $\backslash \backslash Q$

\section{TS: $\mathrm{CN}^{-} \cdot . .9 \mathrm{a}$}

$1 \backslash 1 \backslash G I N C-S C 17 \backslash F O p t \backslash U B 3 L Y P \backslash 6-31 G(d, p) \backslash C 3 H 3 N 102(1-, 2) \backslash G M S 501 \backslash 11-J u n-2004$ $\backslash 0 \backslash \backslash \# P$ B3LYP/6-31G** OPT=TIGHT FREQ=NORAMAN MAXDISK=45875200 GFINPUT I


$82415169,0 . \backslash 0,-1.4494398123,-0.6353799831,0 . \backslash 0,1.4494398123,-0.6353799$ $831,0 . \backslash \mathrm{C},-0.7135647401,-1.6655659781,0 . \backslash \mathrm{C}, 0.7135647401,-1.6655659781,0$ $. \backslash \mathrm{H},-1.1754201629,-2.6836370835,0 . \backslash \mathrm{H}, 1.1754201629,-2.6836370835,0 . \backslash \mathrm{C}, 0$ $., 2.0353249346,0 . \backslash \mathrm{N}, 0 ., 3.195707787,0 . \backslash \backslash$ Version=DEC-AXP-OSF / 1-G03RevB. 0 $5 \backslash$ State $=2-B 1 \backslash \mathrm{HF}=-321.3072217 \backslash \mathrm{S} 2=0.756428 \backslash \mathrm{S} 2-1=0 . \backslash \mathrm{S} 2 \mathrm{~A}=0.750016 \backslash \mathrm{RMSD}=4.3$ $48 e-09 \backslash \mathrm{RMSF}=8.208 \mathrm{e}-07 \backslash \mathrm{Dipole}=0 .,-0.8282013,0 . \backslash \mathrm{PG}=\mathrm{C} 02 \mathrm{~V} \quad[\mathrm{C} 2(\mathrm{H} 1 \mathrm{C} 1 \mathrm{~N} 1), \mathrm{SGV}($ $\mathrm{C} 2 \mathrm{H} 2 \mathrm{O} 2$ ) $] \backslash \backslash @$ 


\section{$\mathrm{HCOO}^{-} \cdot . .9 \mathrm{a}$}

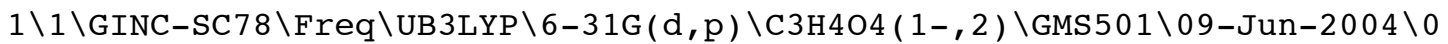
$\backslash \backslash \# P$ B3LYP $/ 6-31 \mathrm{G} * *$ MAXDISK $=45875200$ FREQ=NORAMAN IOP $(6 / 7=3)$ GFINPUT $\backslash \backslash f$

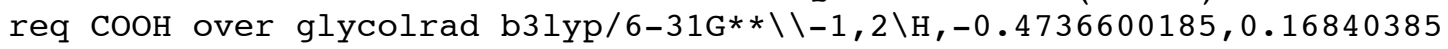
$19,-0.5047700926 \backslash 0,0.4961149815,1.2029838519,-0.3693860926 \backslash \mathrm{C}, 1.7079059$ $815,0.9797198519,-0.0174120926 \backslash C, 2.3329429815,-0.2731181481,0.25947690$ $74 \backslash 0,1.8175789815,-1.4150841481,0.2106959074 \backslash \mathrm{H}, 2.3757389815,1.85770585$ $19,0.0901039074 \backslash \mathrm{H}, 3.4104059815,-0.1503901481,0.5464669074 \backslash 0,-2.6111920$ $185,0.4097708519,0.8172469074 \backslash \mathrm{C},-2.3472170185,-0.3915511481,-0.0650050$ $926 \backslash 0,-1.2499730185,-0.5243631481,-0.7592420926 \backslash \mathrm{H},-3.0945080185,-1.152$ $4821481,-0.3886760926 \backslash \backslash$ Version=DEC-AXP-OSF $/ 1-G 03$ RevB.05 $\backslash$ State $=2-A \backslash H F=-$ $417.6499011 \backslash \mathrm{S} 2=0.756723 \backslash \mathrm{S} 2-1=0 . \backslash \mathrm{S} 2 \mathrm{~A}=0.750018 \backslash \mathrm{RMSD}=6.590 \mathrm{e}-09 \backslash \mathrm{RMSF}=7.276$ e-06\Dipole $=-0.1120839,0.1750393,-0.3008685 \backslash P G=C 01 \quad[\mathrm{X}(\mathrm{C} 3 \mathrm{H} 4 \mathrm{O} 4)] \backslash \backslash @$

\section{TS:HCOO-...9a}

$1 \backslash 1 \backslash G I N C-S C 160 \backslash F T S \backslash U B 3 L Y P \backslash 6-31 G(d, p) \backslash C 3 H 4 O 4(1-, 2) \backslash G M S 501 \backslash 09-J u n-2004 \backslash 0$ $\backslash \backslash \# \mathrm{P}$ B3LYP $/ 6-31 \mathrm{G} *$ * OPT $=(\mathrm{CALCFC}, \mathrm{TS}, \mathrm{NOEIGEN}, \mathrm{TIGHT}, \mathrm{MAXCYCLE}=100)$ MAXDISK= 45875200 FREQ=NORAMAN IOP $(6 / 7=3)$ GFINPUT $\backslash \backslash C S$ COOH orthogonal semi


$2,-0.9320107362,0.4134132472 \backslash 0,-1.4273398642,-0.9320107362,0.413413247$ $2 \backslash C, 0.7126615025,-1.9764993597,0.4546751106 \backslash C,-0.7126615025,-1.9764993$ $597,0.4546751106 \backslash \mathrm{H}, 1.1909339909,-2.9844259446,0.4955883623 \backslash \mathrm{H},-1.190933$ $9909,-2.9844259446,0.4955883623 \backslash 0,0 ., 1.5272073989,0.5255020271 \backslash \mathrm{C}, 0 ., 2$. $2471629495,-0.5756118647 \backslash 0,0 \ldots, 1.8796448318,-1.732901113 \backslash \mathrm{H}, 0 ., 3.3236628$ $19,-0.2996714957 \backslash \backslash$ Version=DEC-AXP-OSF $/ 1-G 03 R e v B .05 \backslash$ State $=2-A^{\prime} \backslash H F=-417$. $6428132 \backslash \mathrm{S} 2=0.756437 \backslash \mathrm{S} 2-1=0 . \backslash \mathrm{S} 2 \mathrm{~A}=0.750016 \backslash \mathrm{RMSD}=6.113 \mathrm{e}-09 \backslash \mathrm{RMSF}=1.531 \mathrm{e}-06$ $\backslash$ Dipole $=0,0.6222657,0.1808223 \backslash P G=C S \quad[S G(C 1 H 2 O 2), x(C 2 H 2 O 2)] \backslash \backslash @$

\section{$\mathrm{CHONH}_{2} \cdots 9 \mathrm{a}$}


$\backslash \#$ B3LYP/6-31G** OPT FREQ=NORAMAN \glyoxal rad anion and formamide sin

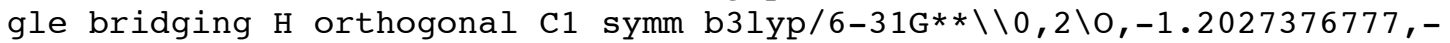
$1.5313419975,-0.1485970243 \backslash 0,-1.1509628084,1.378182741,-0.0159155589 \backslash \mathrm{C}$ $,-2.165634263,-0.7955665447,-0.4631213348 \backslash \mathrm{C},-2.1652396422,0.6232763283$ $,-0.4057398929 \backslash \mathrm{H},-3.110703356,-1.2393375906,-0.8192275253 \backslash \mathrm{H},-3.0465145$ $155,1.1884746575,-0.694928857 \backslash \mathrm{N}, 1.1386229678,-0.189001392,0.7462860118$ $\backslash \mathrm{C}, 2.230590764,-0.0106880864,-0.0843186278 \backslash 0,3.266152427,0.5270508947$, $0.2442408445 \backslash \mathrm{H}, 2.037951975,-0.3819552476,-1.1092440664 \backslash \mathrm{H},-0.3533686998$ $, 0.8440565566,0.2379897951 \backslash \mathrm{H}, 0.4669362371,-0.9275198749,0.5106836295 \backslash \mathrm{H}$ $, 1.3374209045,-0.0539720453,1.7319779842 \backslash \backslash$ Version=Al64T-G03RevC.02\Sta $\mathrm{te}=2-\mathrm{A} \backslash \mathrm{HF}=-398.3271543 \backslash \mathrm{S} 2=0.758035 \backslash \mathrm{S} 2-1=0 . \backslash \mathrm{S} 2 \mathrm{~A}=0.750025 \backslash \mathrm{RMSD}=8.550 \mathrm{e}-09$ $\backslash \mathrm{RMSF}=2.923 \mathrm{e}-05 \backslash \mathrm{Dipole}=-1.3719846,-0.1277639,0.0047522 \backslash \mathrm{PG}=\mathrm{C} 01 \quad[\mathrm{X}(\mathrm{C} 3 \mathrm{H} 6 \mathrm{~N}$ $103)] \backslash \backslash @$

\section{TS: $\mathrm{CHONH}_{2} \cdots 9 \mathrm{a}$}

$1 \backslash 1 \backslash G I N C-S C 88 \backslash F T S \backslash U B 3 L Y P \backslash 6-31 G(d, p) \backslash C 3 H 6 N 103(2) \backslash G M S 501 \backslash 31-M a r-2005 \backslash 0 \backslash \backslash$ \# B3LYP/6-31G** OPT=(TIGHT, TS, CALCFC, NOEIGEN) FREQ=NORAMAN $\backslash$ glyoxalra

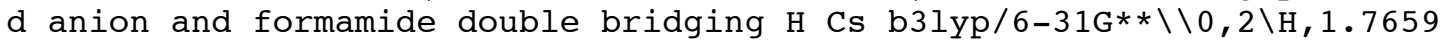
$666933,1.3142480578,0 . \backslash \mathrm{C}, 0.8129819677,1.8735360001,0 . \backslash \mathrm{N},-0.3076881296$, $1.0164169462,0 . \backslash \mathrm{H},-1.167501582,1.566019934,0 . \backslash \mathrm{H},-0.2968213776,0.136135$ $5638,0.8333345277 \backslash \mathrm{H},-0.2968213776,0.1361355638,-0.8333345277 \backslash 0,0.75013$ $3436,3.0793821253,0 . \backslash 0,-0.2909891156,-0.9925213224,-1.4798413148 \backslash 0,-0$. $2909891156,-0.9925213224,1.4798413148 \backslash \mathrm{C},-0.2912103471,-2.0211063637,-0$ $.7102462907 \backslash \mathrm{C},-0.2912103471,-2.0211063637,0.7102462907 \backslash \mathrm{H},-0.2898073645$ $,-3.0050566116,-1.1919676862 \backslash \mathrm{H},-0.2898073645,-3.0050566116,1.191967686$ $2 \backslash \backslash$ Version $=A 164 \mathrm{~T}-\mathrm{G} 03$ RevC. 02 $\backslash$ State $=2-\mathrm{A}^{\prime} \backslash \mathrm{HF}=-398.3086172 \backslash \mathrm{S} 2=0.75408 \backslash \mathrm{S} 2-1$ $=0 . \backslash \mathrm{S} 2 \mathrm{~A}=0.750009 \backslash \mathrm{RMSD}=7.072 \mathrm{e}-09 \backslash \mathrm{RMSF}=5.512 \mathrm{e}-07 \backslash \mathrm{Dipole}=-0.2222382,-0.87$ $20213,0 . \backslash P G=C S \quad[S G(C 1 H 2 N 1 O 1), X(C 2 H 4 O 2)] \backslash \backslash @$ 


\section{Imidazole•..9a}

$1 \backslash 1 \backslash G I N C-S C 62 \backslash F r e q \backslash U B 3 L Y P \backslash 6-31 G(d, p) \backslash C 5 H 7 N 2 O 2(2) \backslash G M S 501 \backslash 09-J u n-2004 \backslash 0 \backslash$ $\backslash \#$ P B 3LYP/6-31G(D,P) MAXDISK=45875200 GFINPUT IOP (6/7=3) FREQ=NORAMAN $\backslash$ $\backslash$ freq ethanaldehyde radical Cs Base=HIS structure is Cs b3lyp/6-31G** \ $\backslash 0,2 \backslash \mathrm{H}, 1.1945638806,-0.2334930149,0 . \backslash \mathrm{O}, 2.0755378806,-0.7570950149,0 . \backslash \mathrm{C}$ $, 1.9438858806,-2.0654850149,0 . \backslash \mathrm{C}, 0.7176778806,-2.7818650149,0 . \backslash 0,-0.42$ $04781194,-2.2667970149,0 . \backslash \mathrm{H}, 2.8857748806,-2.6115680149,0 . \backslash \mathrm{H}, 0.83933188$ $06,-3.8824810149,0 . \backslash \mathrm{N},-0.0000001194,0.8962179851,0 . \backslash \mathrm{C},-1.2813291194,0$. $5804799851,0 . \backslash \mathrm{N},-2.0469311194,1.7028219851,0 . \backslash \mathrm{C},-1.2024511194,2.796657$ $9851,0 . \backslash \mathrm{C}, 0.0629638806,2.2737369851,0 . \backslash \mathrm{H},-1.6583321194,-0.4309490149,0$ . $\backslash \mathrm{H},-3.0548891194,1.7276249851,0 . \backslash \mathrm{H},-1.5702671194,3.8102709851,0 . \backslash \mathrm{H}, 1$. $0073738806,2.7973019851,0 . \backslash \backslash$ Version=DEC-AXP-OSF /1-G03RevB .05 $\backslash$ State $=2-A$ " $\backslash \mathrm{HF}=-454.6621648 \backslash \mathrm{S} 2=0.758024 \backslash \mathrm{S} 2-1=0 . \backslash \mathrm{S} 2 \mathrm{~A}=0.750024 \backslash \mathrm{RMSD}=7.291 \mathrm{e}-09 \backslash \mathrm{RMSF}$ $=3.114 \mathrm{e}-06 \backslash \mathrm{Dipole}=-1.0144976,1.177495,0 . \backslash \mathrm{PG}=\mathrm{CS} \quad[\mathrm{SG}(\mathrm{C} 5 \mathrm{H} 7 \mathrm{~N} 2 \mathrm{O} 2)] \backslash \backslash @$

\section{TS:Imidazole $\cdot .9 a$}


\# B3LYP/6-31G (D,P) OPT=(TS, NOEIGEN, MAXCYCLE=100, CALCFC) FREQ=NORAMAN $\backslash \backslash$

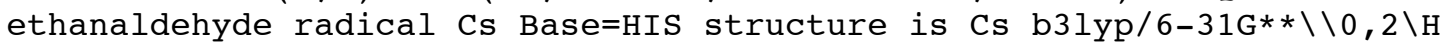
, 0 . $,-0.4303325219,-0.0155424577 \backslash 0,0 \ldots,-1.649938333,1.4092801188 \backslash \mathrm{C}, 0 .,-2$ $.7131575442,0.7121461798 \backslash \mathrm{C}, 0 .,-2.6812684687,-0.7102102394 \backslash 0,0 .,-1.5815$ $268532,-1.3585039891 \backslash \mathrm{H}, 0$. $,-3.7026721173,1.20437125 \backslash \mathrm{H}, 0$. , -3.6422122205 , $-1.2532441115 \backslash \mathrm{N}, 0.0 .6462864074,0.0142869945 \backslash \mathrm{C}, 0$. , $1.3918987184,-1.0744$

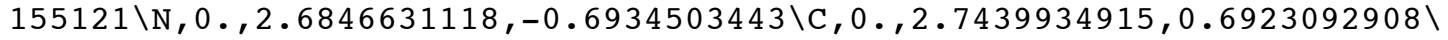
$\mathrm{C}, 0 ., 1.4480308319,1.1261128073 \backslash \mathrm{H}, 0 ., 1.0043184059,-2.0805033487 \backslash \mathrm{H}, 0 ., 3$.

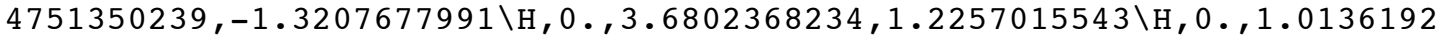
$883,2.1122641654 \backslash \backslash$ Version=Al64T-G03RevC.02 \State=2-A" $\backslash H F=-454.637759 \backslash S$ $2=0.755207 \backslash \mathrm{S} 2-1=0 . \backslash \mathrm{S} 2 \mathrm{~A}=0.750013 \backslash \mathrm{RMSD}=8.137 \mathrm{e}-09 \backslash \mathrm{RMSF}=4.570 \mathrm{e}-05 \backslash \mathrm{Dipole}=0$ $., 3.8563945,-0.5670298 \backslash P G=C S \quad[S G(C 5 H 7 N 2 O 2)] \backslash \backslash @$

\section{$7 \mathrm{~b}$}

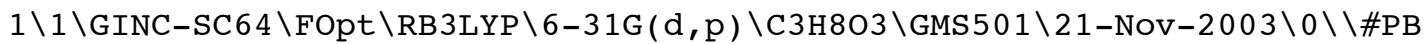
3LYP $/ 6-31 \mathrm{G}(\mathrm{D}, \mathrm{P})$ OPT $=(\mathrm{TIGHT}, \mathrm{MAXCYC}=300)$ FREQ TEST MAXDISK=26214400GFIN

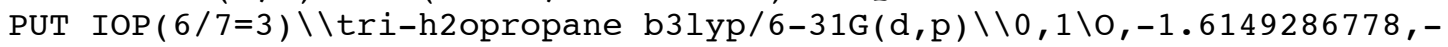
$1.1739308583,0.001837947 \backslash \mathrm{C},-0.1922920573,-1.2033130838,0.0258119305 \backslash \mathrm{C}$, $0.3352190572,0.2369371425,0.0252816046 \backslash \mathrm{H}, 0.1019174094,-1.7074520027,0$. $9497574602 \backslash \mathrm{H}, 0.2203425696,-1.7561822546,-0.8289245935 \backslash 0,-0.2468787928$, $0.9551829598,1.0857878581 \backslash 0,-0.116492638,0.7650291111,-1.2296824835 \backslash \mathrm{C}$, $1.8465025552,0.3456720253,0.1562342929 \backslash \mathrm{H},-1.8395096197,-0.6802346036,-$ $0.802733017 \backslash \mathrm{H},-1.1607033173,0.6256234011,1.1410970385 \backslash \mathrm{H},-0.0974097055$, $1.7277721007,-1.1350734479 \backslash \mathrm{H}, 2.1404390916,1.3997021758,0.1639298383 \backslash \mathrm{H}$, $2.1876751624,-0.1077077427,1.0909301082 \backslash \mathrm{H}, 2.3370719484,-0.1475472785,-$ $0.6864969278 \backslash \backslash$ Version=DEC-AXP-OSF $/ 1-G 03$ RevB .03 $\backslash$ State $=1-A \backslash H F=-344.81400$ $34 \backslash \mathrm{RMSD}=7.626 \mathrm{e}-09 \backslash \mathrm{RMSF}=2.635 e-06 \backslash \mathrm{Dipole}=0.2525526,0.2013185,-0.2888805$ $\backslash \mathrm{PG}=\mathrm{C} 01 \quad[\mathrm{X}(\mathrm{C} 3 \mathrm{H} 8 \mathrm{O} 3)] \backslash \backslash @$ 


\section{7b*}

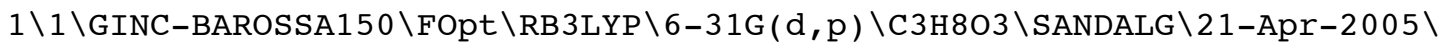
$0 \backslash \backslash \#$ B3LYP/6-31G(D,P) OPT FREQ=NORAMAN \\gem-diol C1 nonrad triolpropan


$929479,-0.3793740933,-0.431706 \backslash 0,1.7388200731,0.5320867611,-0.512478 \backslash \mathrm{C}$ $,-0.6663409507,0.3585640916,-0.387483 \backslash \mathrm{H}, 0.761326858,-1.0332761046,-1.3$ $10266 \backslash \mathrm{H}, 1.6710358097,-1.3852192296,0.88632 \backslash \mathrm{H}, 1.4761591745,1.2699867972$ $, 0.06568 \backslash \mathrm{O},-0.6080888245,1.2768910836,0.710485 \backslash \mathrm{H},-0.4603369004,0.72487$ $10633,1.495003 \backslash \mathrm{C},-1.8750450778,-0.5662827424,-0.305412 \backslash \mathrm{H},-0.7278718648$ $, 0.9840741,-1.284288 \backslash \mathrm{H},-2.7939929968,0.0231293839,-0.250682 \backslash \mathrm{H},-1.93048$ $81667,-1.2131007347,-1.1879 \backslash \mathrm{H},-1.811140166,-1.2078917511,0.578205 \backslash \backslash$ Ver sion=IA32L-G03RevC . 02 \State $=1-A \backslash H F=-344.8118174 \backslash$ RMSD=6 . 255e-09 $\backslash$ RMSF $=3$. $486 \mathrm{e}-06 \backslash \mathrm{Dipole}=-0.0198762,-0.4194854,0.120027 \backslash \mathrm{PG}=\mathrm{C} 01[\mathrm{X}(\mathrm{C} 3 \mathrm{H} 8 \mathrm{O} 3)] \backslash \backslash @$

\section{8b}

$1 \backslash 1 \backslash G I N C-S C 64 \backslash F O p t \backslash U B 3 L Y P \backslash 6-31 G(d, p) \backslash C 3 H 7 O 3(2) \backslash G M S 501 \backslash 22-N o v-2003 \backslash 0 \backslash \backslash \#$ P B3LYP/6-31G(D,P) OPT=(TIGHT,READFC) FREQ TEST MAXDISK=26214400 GUESS =READ GFINPUT IOP (6/7=3) \\tri-h2opropane radicale/short/k29/gms501/r-3

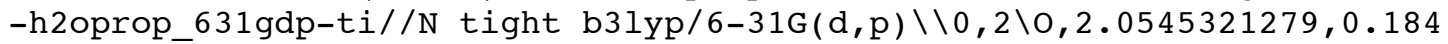
$7235612,-0.0380162098 \backslash \mathrm{C}, 0.8655817524,0.8532467011,0.025248293 \backslash \mathrm{C},-0.378$ $9056672,0.0141466597,0.0082436475 \backslash \mathrm{H}, 0.9027117324,1.7673056237,0.607971$ $0061 \backslash 0,-0.6755529453,-0.5541258373,1.2877776872 \backslash 0,-0.081334592,-1.0491$ $143427,-0.9072462829 \backslash \mathrm{C},-1.6229997732,0.7844605323,-0.4099211614 \backslash \mathrm{H}, 1.85$ $84909494,-0.6288659995,-0.5383786147 \backslash \mathrm{H}, 0.1663123597,-0.8499269994,1.66$ $46119831 \backslash \mathrm{H},-0.6946703088,-1.7652710718,-0.6906569154 \backslash \mathrm{H},-2.50195327,0.1$ $373523647,-0.3324493193 \backslash \mathrm{H},-1.775807038,1.6452976471,0.2467801924 \backslash \mathrm{H},-1$. $518299021,1.1311180268,-1.4394245624 \backslash \backslash$ Version=DEC-AXP-OSF / 1-G03RevB. 03 $\backslash$ State $=2-\mathrm{A} \backslash \mathrm{HF}=-344.1509259 \backslash \mathrm{S} 2=0.753345 \backslash \mathrm{S} 2-1=0 . \backslash \mathrm{S} 2 \mathrm{~A}=0.750008 \backslash \mathrm{RMSD}=2.134$ e-09 $\backslash \mathrm{RMSF}=1.213 \mathrm{e}-06 \backslash \mathrm{Dipole}=-0.3238251,-0.4382651,0.0685728 \backslash \mathrm{PG}=\mathrm{C} 01[\mathrm{X}(\mathrm{C}$ $3 \mathrm{H} 703$ ) $] \backslash \backslash \mathrm{Q}$

\section{8b*}

$1 \backslash 1 \backslash G I N C-B A R O S S A 042 \backslash F O p t \backslash U B 3 L Y P \backslash 6-31 G(d, p) \backslash C 3 H 7 O 3(2) \backslash S A N D A L G \backslash 21-A p r-20$ $05 \backslash 0 \backslash \backslash \#$ B3LYP/6-31G(D,P) OPT FREQ=NORAMAN \gem-diol C1 nonrad triolpro

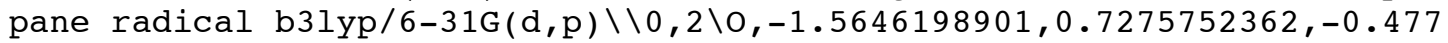
$92 \backslash C,-0.6561280553,-0.3660489009,-0.407886 \backslash 0,-1.028415187,-1.238831844$ $7,0.657956 \backslash \mathrm{C}, 0.7064340355,0.2354458933,-0.251479 \backslash \mathrm{H},-0.7176941504,-0.99$ $60798916,-1.303644 \backslash \mathrm{H},-2.4436829452,0.363063369,-0.305073 \backslash \mathrm{H},-0.78817811$ $89,-0.787827881,1.481734 \backslash 0,0.7602132002,1.3261608852,0.582985 \backslash \mathrm{H},-0.109$ $8707349,1.7558820166,0.502378 \backslash \mathrm{C}, 1.9371939093,-0.6005382925,-0.27748 \backslash \mathrm{H}$, $2.0862978242,-1.164522315,0.658072 \backslash \mathrm{H}, 1.8883357993,-1.3293972851,-1.092$ $32 \backslash \mathrm{H}, 2.822363004,0.0264895739,-0.424248 \backslash \backslash$ Version=IA32L-G03RevC.02\Stat $\mathrm{e}=2-\mathrm{A} \backslash \mathrm{HF}=-344.1525801 \backslash \mathrm{S} 2=0.753382 \backslash \mathrm{S} 2-1=0 . \backslash \mathrm{S} 2 \mathrm{~A}=0.750008 \backslash \mathrm{RMSD}=6.540 \mathrm{e}-09 \backslash$ $\mathrm{RMSF}=3.172 \mathrm{e}-06 \backslash \mathrm{Dipole}=-0.4371544,-0.1010864,0.1366652 \backslash \mathrm{PG}=\mathrm{C} 01] \mathrm{X}(\mathrm{C} 3 \mathrm{H} 7 \mathrm{O} 3$ )$] \backslash \$

\section{9b}

$1 \backslash 1 \backslash G I N C-L C 139 \backslash F O p t \backslash U B 3 L Y P \backslash 6-31 G(d, p) \backslash C 3 H 5 O 2(2) \backslash G M S 501 \backslash 21-N o v-2003 \backslash 0 \backslash \backslash$ \#P B3LYP/6-31G(D,P) OPT= (CALCFC, TIGHT) MAXDISK=26214400 GFINPUTIOP (6/) 7=3) FREQ TEST \\propolrade/short/k29/gms501/propolrad-C1-631gdp//N \\0, $2 \backslash \mathrm{H}, 1.1822056939,-1.4344498373,0 . \backslash \mathrm{O}, 0.3029447486,-1.8870341072,0 . \backslash \mathrm{C},-0$ $.555307303,-0.8704530924,0 . \backslash \mathrm{C},-0.0000289463,0.4469519777,0 . \backslash 0,1.249885$ $9501,0.5333810538,0 . \backslash \mathrm{C},-0.8927054782,1.6638779818,0 . \backslash \mathrm{H},-1.6057471134,-$ $1.1342197997,0 . \backslash \mathrm{H},-1.954426924,1.4022006385,0 . \backslash \mathrm{H},-0.6782134405,2.27671$ $61115,0.8812071012 \backslash \mathrm{H},-0.6782134405,2.2767161115,-0.8812071012 \backslash \backslash$ Version 
$=\mathrm{x} 86-\mathrm{Linux}-\mathrm{G} 03$ RevB $.03 \backslash$ State $=2-\mathrm{A} " \backslash \mathrm{HF}=-267.7445416 \backslash \mathrm{S} 2=0.757743 \backslash \mathrm{S} 2-1=0 . \backslash \mathrm{S}$ $2 \mathrm{~A}=0.750025 \backslash \mathrm{RMSD}=3.603 e-09 \backslash \mathrm{RMSF}=1.102 \mathrm{e}-06 \backslash \mathrm{Dipole}=-0.8777158,0.117774,0$ $. \backslash \mathrm{PG}=\mathrm{CS}[\mathrm{SG}(\mathrm{C} 3 \mathrm{H} 3 \mathrm{O} 2), \mathrm{X}(\mathrm{H} 2)] \backslash \backslash @$

\section{$9 b^{*}$}

$1 \backslash 1 \backslash G I N C-L C 139 \backslash F O p t \backslash U B 3 L Y P \backslash 6-31 G(d, p) \backslash C 3 H 5 O 2(2) \backslash G M S 501 \backslash 21-N o v-2003 \backslash 0 \backslash \backslash$ \#P B3LYP/6-31G (D,P) OPT=(TIGHT, MAXCYC=300) MAXDISK=26214400GFINPUTIO $\mathrm{P}(6 / 7=3)$ FREQ TEST \\propolradi@/short/k29/gms501/propolrad-C2-631gdp// $\mathrm{N} \backslash \backslash 0,2 \backslash \mathrm{H},-0.9369801732,-1.3430143134,0 . \backslash 0,0.049753018,-1.3665700649,0$. $\backslash C, 0.3920547249,-0.0753120674,0 . \backslash \mathrm{C},-0.690893315,0.8480135965,0 . \backslash 0,-1.8$ $653531218,0.4131855833,0 . \backslash \mathrm{C}, 1.8418343633,0.2424771312,0 . \backslash \mathrm{H},-0.46389763$ $69,1.9255118829,0 . \backslash \mathrm{H}, 1.9987201148,1.3237748419,0 . \backslash \mathrm{H}, 2.3344919431,-0.18$ $513426,0.8816616277 \backslash \mathrm{H}, 2.3344919431,-0.18513426,-0.8816616277 \backslash \backslash$ Version= $\mathrm{x} 86-\mathrm{Linux}-\mathrm{G} 03 \mathrm{RevB} .03 \backslash \mathrm{State}=2-\mathrm{A} " \backslash \mathrm{HF}=-267.7460869 \backslash \mathrm{S} 2=0.758059 \backslash \mathrm{S} 2-1=0 . \backslash \mathrm{S} 2$ $\mathrm{A}=0.750024 \backslash \mathrm{RMSD}=6.483 e-09 \backslash \mathrm{RMSF}=1.790 \mathrm{e}-06 \backslash \mathrm{Dipole}=1.0418543,0.2184388,0$. $\backslash \mathrm{PG}=\mathrm{CS} \quad[\mathrm{SG}(\mathrm{C} 3 \mathrm{H} 3 \mathrm{O} 2), \mathrm{X}(\mathrm{H} 2)] \backslash \backslash @$

\section{0b}

$1 \backslash 1 \backslash G I N C-L C 76 \backslash F T S \backslash U B 3 L Y P \backslash 6-31 G(d, p) \backslash C 3 H 5 O 2(2) \backslash G M S 501 \backslash 21-N o v-2003 \backslash 0 \backslash \backslash \# P$ B3LYP /6-31G (D , P ) OPT= (CALCFC, TS , NOEIGEN , TIGHT , MAXCYCLE=200) MAXDISK=2 6214400 FREQ TEST \\propyl-semidione b3lyp/6-31G(d,p) Cs symmetry $\backslash \backslash 0,2 \backslash$ $\mathrm{H},-0.181216444,-1.4722327681,0 . \backslash \mathrm{O}, 1.0617794362,-1.4782838629,0 . \backslash 0,-1.1$ $096694439,-0.6275113757,0 . \backslash C, 1.0736491339,-0.1873523668,0 . \backslash C,-0.254830$ $4914,0.3392965336,0 . \backslash \mathrm{H}, 2.0024619654,0.3762631665,0 . \backslash \mathrm{C},-0.6659508121,1$. $7701385417,0 . \backslash \mathrm{H}, 0.203079395,2.4331566758,0 . \backslash \mathrm{H},-1.2792059186,1.98833929$ $17,-0.8812181367 \backslash \mathrm{H},-1.2792059186,1.9883392917,0.8812181367 \backslash \backslash$ Version=x

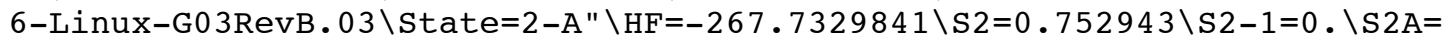
$0.750006 \backslash \mathrm{RMSD}=9.309 \mathrm{e}-09 \backslash \mathrm{RMSF}=1.039 \mathrm{e}-06 \backslash \mathrm{Dipole}=0.2131656,1.1564879,0 . \backslash \mathrm{P}$ $\mathrm{G}=\mathrm{CS}[\mathrm{SG}(\mathrm{C} 3 \mathrm{H} 3 \mathrm{O} 2), \mathrm{X}(\mathrm{H} 2)] \backslash \backslash @$

\section{$11 b$}

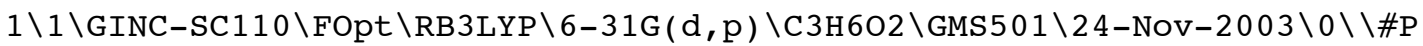
B3LYP $/ 6-31 \mathrm{G}(\mathrm{D}, \mathrm{P})$ OPT $=(\mathrm{MAXCYC}=100, \mathrm{READFC}, \mathrm{TIGHT}) \mathrm{MAXDISK}=26214400 \mathrm{GUESS}=$ READ GFINPUT IOP $(6 / 7=3)$ FREQ TEST \\1-OHpropanone b3lyp/6-31G(d,p)C1 ymmetry $\backslash \backslash 0,1 \backslash \mathrm{H},-1.6679996563,0.8656478849,0.0097734789 \backslash 0,-1.9318559516$ $,-0.0725810781,0.0316769615 \backslash \mathrm{C},-0.728772293,-0.7823944586,-0.0263889671$ $\backslash \mathrm{C}, 0.473091638,0.155654242,-0.0123192509 \backslash 0,0.2779572516,1.3596845751,-$ $0.012889961 \backslash \mathrm{C}, 1.856043983,-0.4499686417,0.0142871141 \backslash \mathrm{H}, 2.600238214,0.3$ $137094375,-0.2150081681 \backslash \mathrm{H}, 2.0574989711,-0.8524883869,1.0142953227 \backslash \mathrm{H}, 1$. $93925139,-1.2825744089,-0.691682448 \backslash \mathrm{H},-0.6368182781,-1.4832384211,0.82$ $0021608 \backslash \mathrm{H},-0.6631610088,-1.3976309317,-0.9411691743 \backslash \backslash$ Version=DEC $-\mathrm{AXP}-\mathrm{O}$ $\mathrm{SF} / 1-\mathrm{G} 03 \mathrm{RevB} .03 \backslash \mathrm{State}=1-\mathrm{A} \backslash \mathrm{HF}=-268.3758015 \backslash \mathrm{RMSD}=3.083 \mathrm{e}-09 \backslash \mathrm{RMSF}=1.609 \mathrm{e}-0$ $6 \backslash \mathrm{Dipole}=0.9592397,-0.7392162,-0.0062078 \backslash \mathrm{PG}=\mathrm{C} 01[\mathrm{X}(\mathrm{C} 3 \mathrm{H} 6 \mathrm{O} 2)] \backslash \backslash @$

\section{$11 b^{*}$}

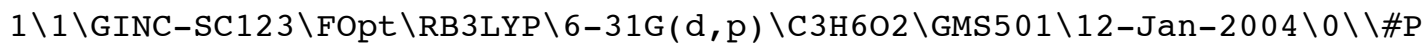
$\mathrm{B} 3 \mathrm{LYP} / 6-31 \mathrm{G}(\mathrm{D}, \mathrm{P})$ OPT $=(\mathrm{MAXCYC}=100, \mathrm{CALCFC})$ MAXDISK=26214400GF INPUTIOP (

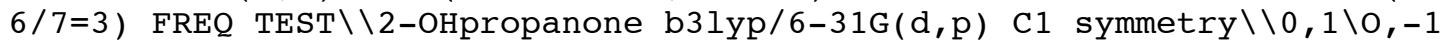
$.1864297569,-1.3754998698,-0.3916413404 \backslash \mathrm{C},-0.4142179748,-0.9327962686$, $0.4343457413 \backslash \mathrm{C}, 0.1056873922,0.4895984878,0.3580936676 \backslash \mathrm{C}, 1.6400909537,0$ $.5108406552,0.3618522834 \backslash \mathrm{H}, 1.9858141181,1.5461330365,0.3190733913 \backslash \mathrm{H}, 2$. $0437730749,0.047715862,1.2685785891 \backslash \mathrm{H}, 2.029879845,-0.0172078708,-0.513$ $1741023 \backslash \mathrm{H},-0.056654082,-1.5486445916,1.2848356085 \backslash 0,-0.4047294186,1.13$ $53743072,-0.779890088 \backslash \mathrm{H},-0.2492563163,0.9900956408,1.2796536277 \backslash \mathrm{H},-1.0$ $136454618,0.4970551784,-1.1924658408 \backslash \backslash$ Version=DEC-AXP-OSF / 1-G03RevB.03 $\backslash$ State $=1-\mathrm{A} \backslash \mathrm{HF}=-268.3680132 \backslash \mathrm{RMSD}=4.094 \mathrm{e}-09 \backslash \mathrm{RMSF}=1.239 \mathrm{e}-05 \backslash \mathrm{Dipole}=0.5239$ $827,-0.1467819,0.7498106 \backslash \mathrm{PG}=\mathrm{C} 01[\mathrm{X}(\mathrm{C} 3 \mathrm{H} 6 \mathrm{O} 2)] \backslash \backslash @$ 


\section{2}


$\mathrm{P}$ B3LYP/6-31G** OPT $=($ TIGHT, MAXCYCLE $=100) \quad$ FREQ=NORAMAN MAXDISK $=32768000$ GFINPUT IOP $(6 / 7=3) \backslash \backslash c h l o r o a c e t a l d e h y d e ~ b 3 l y p / 6-31 G * *$ Cs symm $\backslash \backslash 0,1 \backslash 0,2$ $.2945130569,-0.0480626366,-0.1729860216 \backslash \mathrm{C}, 1.1716141417,-0.3487240397,0$ $.1528392011 \backslash \mathrm{C}, 0.0308451997,0.657864379,0.1165121522 \backslash \mathrm{Cl},-1.5681676116,-$ $0.1576116914,-0.0645888786 \backslash \mathrm{H}, 0.172422498,1.3543130972,-0.7104651487 \backslash \mathrm{H}$, $0.0104032356,1.216539392,1.0571639625 \backslash \mathrm{H}, 0.90516316,-1.3617946775,0.519$ $0921744 \backslash \backslash$ Version=DEC $-\mathrm{AXP}-\mathrm{OSF} / 1-\mathrm{G} 03$ RevB. 05 $\backslash$ State $=1-\mathrm{A} \backslash \mathrm{HF}=-613.4232262 \backslash \mathrm{RM}$ $\mathrm{SD}=6.547 \mathrm{e}-09 \backslash \mathrm{RMSF}=4.308 \mathrm{e}-06 \backslash \mathrm{Dipole}=-0.2169865,0.2373442,0.321575 \backslash \mathrm{PG}=\mathrm{C} 0$ $1[\mathrm{X}(\mathrm{C} 2 \mathrm{H} 3 \mathrm{C} 11 \mathrm{O} 1)] \backslash \backslash @$

\section{3}

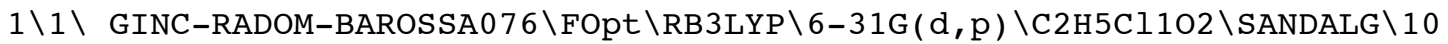

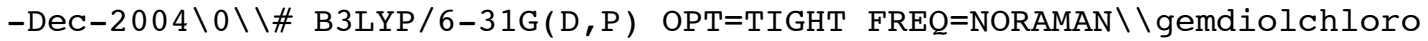

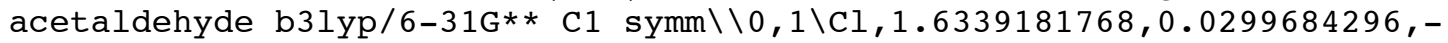
$0.309854584 \backslash \mathrm{C}, 0.3589525473,-0.2823632534,0.9446254548 \backslash \mathrm{C},-1.0445313329$, $-0.0297957372,0.3988813165 \backslash 0,-1.2140221374,1.2594696608,-0.1203292116 \backslash$ $\mathrm{H}, 0.4682455131,-1.3192110742,1.2615181308 \backslash \mathrm{H}, 0.5649129959,0.3949400369$, $1.7730750684 \backslash \mathrm{H},-0.5286853816,1.3812237537,-0.7975730199 \backslash \mathrm{O},-1.317969803$ $,-1.0336812863,-0.5471473818 \backslash \mathrm{H},-1.7387097524,-0.084339127,1.2526448962$ $\backslash \mathrm{H},-2.1729641437,-0.8154299439,-0.943365028 \backslash \backslash$ Version=x86-Linux-G03RevB $.02 \backslash$ State $=1-\mathrm{A} \backslash \mathrm{HF}=-689.8672512 \backslash \mathrm{RMSD}=3.707 \mathrm{e}-09 \backslash \mathrm{RMSF}=1.956 \mathrm{e}-06 \backslash \mathrm{Dipole}=-0$. $5160241,-0.0540685,0.3733429 \backslash \mathrm{PG}=\mathrm{C} 01 \quad[\mathrm{X}(\mathrm{C} 2 \mathrm{H} 5 \mathrm{Cl} 1 \mathrm{O} 2)] \backslash \backslash @$

\section{TS: $13 \rightarrow 14$}


$\backslash 10-D e c-2004 \backslash 0 \backslash \backslash \#$ B3LYP / 6-31G(D,P) OPT= (CALCFC, TS, NOEIGENTEST) FREQ=NO RAMAN \llowest conformer gemdiol chloroacetaldehyde b3lyp/6-31G**C1

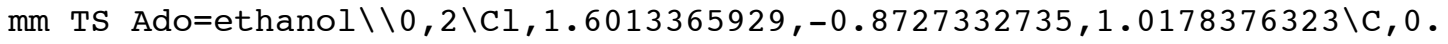
$9825533759,-0.4090061388,-0.5927331208 \backslash \mathrm{C}, 0.9955505124,1.0885231075,-0$. $8387051933 \backslash \mathrm{O}, 0.3045128929,1.8170028517,0.1440516163 \backslash \mathrm{H}, 1.5017694477,-0$. $9837304382,-1.3588155622 \backslash \mathrm{H},-0.3004301804,-0.7880854645,-0.6410333925 \backslash \mathrm{H}$ $, 0.7129133801,1.5927720244,0.9958243074 \backslash 0,2.3381006447,1.4992133945,-0$ $.9576013276 \backslash \mathrm{H}, 0.4380300969,1.2718668654,-1.7700157298 \backslash \mathrm{H}, 2.3262022757,2$ $.458369014,-1.0891599051 \backslash \mathrm{C},-2.3141141396,-0.4509486986,0.4321666168 \backslash \mathrm{C}$, $-1.6151907427,-1.1403631796,-0.7116565708 \backslash \mathrm{H},-2.1574683722,0.6347328917$ $, 0.3612614733 \backslash \mathrm{O},-3.7037542015,-0.7845446179,0.3565888779 \backslash \mathrm{H},-1.58588285$ $88,-2.2289306102,-0.6406151336 \backslash \mathrm{H},-1.9278820343,-0.7988622649,-1.700326$ $9926 \backslash \mathrm{H},-1.8791314221,-0.7850428481,1.3870563022 \backslash \mathrm{H},-4.1545111364,-0.319$ $2270895,1.0738411587 \backslash \backslash$ Version $=x 86-$ Linux-G03RevB. $02 \backslash$ State $=2-A \backslash H F=-844.2$ $194616 \backslash \mathrm{S} 2=0.756832 \backslash \mathrm{S} 2-1=0 . \backslash \mathrm{S} 2 \mathrm{~A}=0.750025 \backslash \mathrm{RMSD}=9.262 \mathrm{e}-09 \backslash \mathrm{RMSF}=6.511 \mathrm{e}-06 \backslash$ Dipole $=-0.0163325,0.6389614,-0.0680949 \backslash \mathrm{PG}=\mathrm{C} 01 \quad[\mathrm{X}(\mathrm{C} 4 \mathrm{H} 10 \mathrm{Cl} 1 \mathrm{O} 3)] \backslash \backslash @$

\section{4}

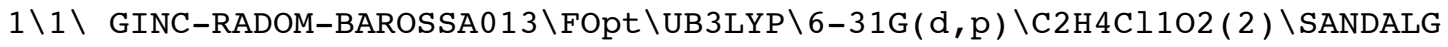

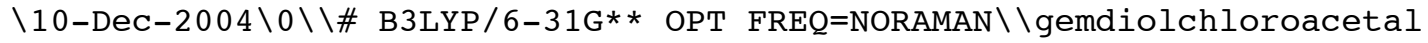

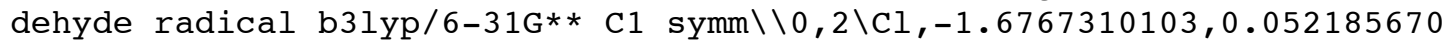
$2,-0.251475 \backslash \mathrm{C},-0.3959049596,-0.2053380779,0.880049 \backslash \mathrm{C}, 1.021511008,-0.04$ $05227991,0.428946 \backslash 0,1.4390722092,-1.0633657169,-0.460991 \backslash \mathrm{H},-0.65141383$ $68,-0.8297911281,1.72676 \backslash \mathrm{H}, 0.8412892025,-1.0293408345,-1.224248 \backslash \mathrm{O}, 1.16$ $39527579,1.2310202289,-0.150561 \backslash \mathrm{H}, 1.6699060333,-0.1692786715,1.307576 \backslash$ $\mathrm{H}, 2.0668087492,1.2751784065,-0.496561 \backslash \backslash$ Version=x86-Linux-G03RevB.02 $\backslash \mathrm{St}$ ate $=2-\mathrm{A} \backslash \mathrm{HF}=-689.1971983 \backslash \mathrm{S} 2=0.753991 \backslash \mathrm{S} 2-1=0 . \backslash \mathrm{S} 2 \mathrm{~A}=0.750011 \backslash \mathrm{RMSD}=3.478 \mathrm{e}-0$ $9 \backslash \mathrm{RMSF}=2.517 \mathrm{e}-06 \backslash \mathrm{Dipole}=0.40458,-0.1885011,0.0717376 \backslash \mathrm{PG}=\mathrm{C} 01[\mathrm{X}(\mathrm{C} 2 \mathrm{H} 4 \mathrm{Cl} 1$ $02)] \backslash \backslash @$ 


\section{5}

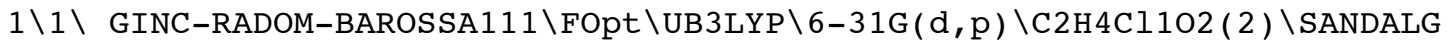

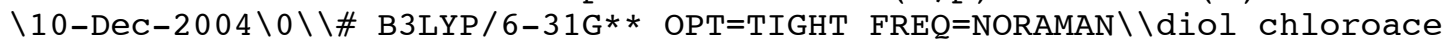

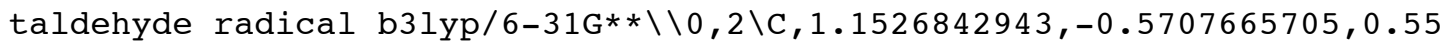
$81770902 \backslash \mathrm{C}, 0.1483072342,0.4020645106,0.6792970054 \backslash 0,1.98025989,-0.5762$ $932454,-0.4852800026 \backslash \mathrm{H}, 1.7745581802,0.2131341116,-1.0208413791 \backslash \mathrm{H}, 1.237$ $1095056,-1.4220918493,1.2200656176 \backslash 0,0.3775109701,1.5076822004,-0.0954$ $238316 \backslash \mathrm{H},-0.4810026398,1.8841619361,-0.3424893748 \backslash \mathrm{Cl},-1.6974846144,-0$. $4498440022,-0.2514231282 \backslash \mathrm{H},-0.3415426533,0.5332445593,1.6382444159 \backslash \backslash \mathrm{Ve}$ rsion $=x 86-$ Linux-G03RevB.02 $\backslash$ State $=2-A \backslash H F=-689.2100055 \backslash S 2=0.757248 \backslash S 2-1=$ $0 . \backslash S 2 A=0.75002 \backslash R M S D=7.490 e-09 \backslash R M S F=5.672 e-06 \backslash D i p o l e=0.9633228,0.761260$ $9,0.5029138 \backslash \mathrm{PG}=\mathrm{C} 01 \quad[\mathrm{X}(\mathrm{C} 2 \mathrm{H} 4 \mathrm{Cl} 1 \mathrm{O} 2)] \backslash \backslash @$

\section{$\mathrm{HCl}$}

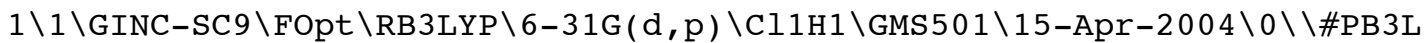
YP/6-31G $(\mathrm{D}, \mathrm{P})$ OPT=TIGHT FREQ=NORAMAN MAXDISK=13107200 GFINPUTIOP $(6 / 7=$ $3) \backslash \backslash \mathrm{HCl} b 3 \mathrm{lyp} / 6-31 \mathrm{G} * * \backslash \backslash 0,1 \backslash \mathrm{Cl},-0.071448445,0 ., 0 . \backslash \mathrm{H}, 1.2146235658,0 ., 0 . \backslash$ $\backslash$ Version=DEC-AXP-OSF $/ 1-G 03 R e v B .05 \backslash$ State $=1-S G \backslash H F=-460.8007767 \backslash \mathrm{RMSD}=7.68$ $5 e-09 \backslash \mathrm{RMSF}=5.814 \mathrm{e}-07 \backslash \mathrm{Dipole}=0.5639801,0 ., 0 . \backslash \mathrm{PG}=\mathrm{C} * \mathrm{~V} \quad[\mathrm{C} *(\mathrm{H} 1 \mathrm{Cl} 1)] \backslash \backslash @$

\section{6}

$1 \backslash 1 \backslash$ GINC-SC1 $11 \backslash$ FOpt $\backslash R B 3 L Y P \backslash 6-31 G(d, p) \backslash C 2 H 2 O 2 \backslash G M S 501 \backslash 13-A p r-2004 \backslash 0 \backslash \backslash \# P$ B3LYP $/ 6-31 \mathrm{G}(\mathrm{D}, \mathrm{P})$ OPT=(TIGHT, MAXCYCLE=200) MAXDISK=32768000GF INPUTIOP

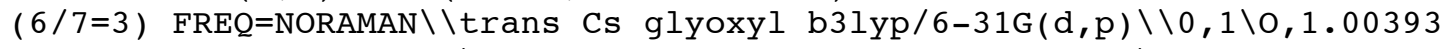
$81474,-1.4140461043,0 . \backslash \mathrm{C},-0.0151149387,-0.7623429198,0 . \backslash \mathrm{C}, 0.0151149387$ $, 0.7623429198,0 . \backslash \mathrm{O},-1.0039381474,1.4140461043,0 . \backslash \mathrm{H}, 1.0323683496,1.2046$ $437656,0 . \backslash \mathrm{H},-1.0323683496,-1.2046437656,0 . \backslash \backslash$ Version=DEC-AXP-OSF / 1-G03R evB . 05 $\backslash$ state $=1-A G \backslash H F=-227.8213601 \backslash R M S D=1.959 e-09 \backslash R M S F=4.596 e-06 \backslash D i p o l e$ $=0,0 ., 0 . \backslash \mathrm{PG}=\mathrm{C} 02 \mathrm{H} \quad[\mathrm{SGH}(\mathrm{C} 2 \mathrm{H} 2 \mathrm{O} 2)] \backslash \backslash @$

\section{7}

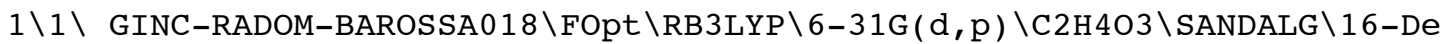

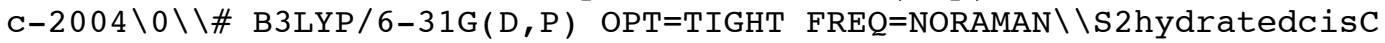

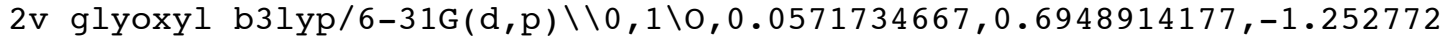
$8371 \backslash \mathrm{C}, 0.0471634032,0.6380137341,0.133394735 \backslash 0,1.3323170155,0.56151278$ $35,0.7220190782 \backslash \mathrm{C},-0.644244082,-0.6407396903,0.6194498113 \backslash \mathrm{H},-0.4944048$ $813,1.5260178751,0.4973724802 \backslash 0,-1.0112855452,-1.4874081141,-0.1638673$ $065 \backslash \mathrm{H},-0.7479224164,-0.7590953196,1.7144272447 \backslash \mathrm{H},-0.0383052892,-0.2215$ $50779,-1.5673981895 \backslash \mathrm{H}, 1.8374771641,1.3190152639,0.3954997105 \backslash \backslash$ Version= $\mathrm{x} 86-$ Linux-G03RevB.02 $\backslash$ State $=1-\mathrm{A} \backslash \mathrm{HF}=-304.2667416 \backslash \mathrm{RMSD}=4.357 \mathrm{e}-09 \backslash \mathrm{RMSF}=2.6$ $76 \mathrm{e}-06 \backslash \mathrm{Dipole}=0.1227961,0.556627,0.4325159 \backslash \mathrm{PG}=\mathrm{C} 01[\mathrm{X}(\mathrm{C} 2 \mathrm{H} 4 \mathrm{O} 3)] \backslash \backslash @$

\section{TS:17 $\rightarrow 18$}

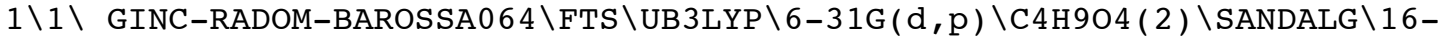

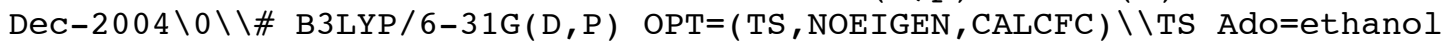

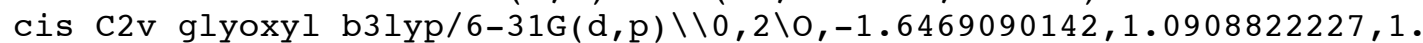
$0237115352 \backslash \mathrm{C},-1.0493173929,0.4414791812,-0.0406027347 \backslash 0,-0.7091424765$, $1.2924592042,-1.0927622212 \backslash \mathrm{C},-1.8847955885,-0.6966629133,-0.552913778 \backslash$ $\mathrm{H},-0.0039907244,-0.0561391413,0.3682652284 \backslash \mathrm{O},-2.8005165393,-1.14013318$ $51,0.1165778115 \backslash \mathrm{H},-1.5912381723,-1.1256453581,-1.528228366 \backslash \mathrm{H},-2.421093$ $0894,0.5469274255,1.2636541286 \backslash \mathrm{H},-0.2927885514,2.0746657328,-0.7039132$ $306 \backslash \mathrm{C}, 2.2954783908,-0.3829204722,-0.3511545425 \backslash \mathrm{C}, 1.4621462156,-0.32382$ $04754,0.8941864698 \backslash \mathrm{H}, 2.1090340134,0.5121172724,-0.9655912089 \backslash \mathrm{H}, 2.00468$ $76505,-1.2564813652,-0.955863035 \backslash 0,3.6733719593,-0.4617713033,0.031504$ $1617 \backslash \mathrm{H}, 1.3225243734,-1.2522614206,1.4455359631 \backslash \mathrm{H}, 1.5963934887,0.553236$ $2915,1.5256357286 \backslash \mathrm{H}, 4.2009698264,-0.4763668666,-0.7788379936 \backslash \backslash$ Version= $\mathrm{x} 86-\mathrm{Linux}-\mathrm{G} 03 \mathrm{RevB} .02 \backslash \mathrm{State}=2-\mathrm{A} \backslash \mathrm{HF}=-458.6284741 \backslash \mathrm{S} 2=0.75786 \backslash \mathrm{S} 2-1=0 . \backslash \mathrm{S} 2 \mathrm{~A}=$ $0.750037 \backslash \mathrm{RMSD}=6.759 \mathrm{e}-09 \backslash \mathrm{RMSF}=4.743 \mathrm{e}-06 \backslash \mathrm{Dipole}=0.5084316,0.1956726,-0.6$ $372687 \backslash \mathrm{PG}=\mathrm{C} 01 \quad[\mathrm{X}(\mathrm{C} 4 \mathrm{H} 9 \mathrm{O} 4)] \backslash \backslash @$ 


\section{8}

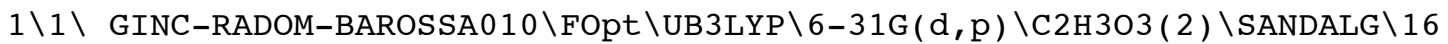

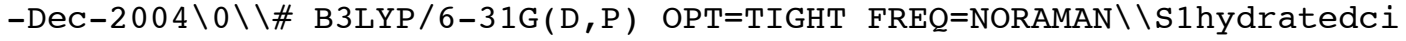

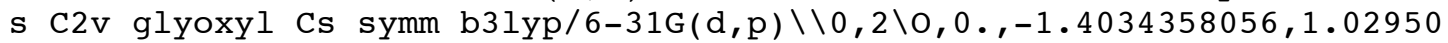
$77879 \backslash \mathrm{C}, 0$. $-0.2643602004,0.3358726591 \backslash 0,0.0 .8718418578,1.0325314789 \backslash \mathrm{C}$ , 0 . $,-0.1873045045,-1.072635987 \backslash \mathrm{O}, 0 ., 0.9641588864,-1.5895691306 \backslash \mathrm{H}, 0 .,-1$ $.1164425899,-1.6560045694 \backslash \mathrm{H}, 0$. , -1.1912226272, $1.9756305334 \backslash \mathrm{H}, 0 ., 1.55713$ $39379,0.3211929132 \backslash \backslash$ Version=x86-Linux-G03RevB.02 $\backslash$ State $=2-A " \backslash H F=-303.64$ $76206 \backslash \mathrm{S} 2=0.756553 \backslash \mathrm{S} 2-1=0 . \backslash \mathrm{S} 2 \mathrm{~A}=0.750016 \backslash \mathrm{RMSD}=9.812 \mathrm{e}-09 \backslash \mathrm{RMSF}=4.116 \mathrm{e}-06 \backslash \mathrm{D}$ ipole $=0,-0.4781314,1.1058047 \backslash \mathrm{PG}=\mathrm{CS} \quad[\mathrm{SG}(\mathrm{C} 2 \mathrm{H} 3 \mathrm{O} 3)] \backslash \backslash @$

\section{9}

$1 \backslash 1 \backslash G I N C-S C 102 \backslash F T S \backslash U B 3 L Y P \backslash 6-31 G(d, p) \backslash C 2 H 303(2) \backslash G M S 501 \backslash 05-J a n-2005 \backslash 0 \backslash \backslash \#$ B3LYP/6-31G(D,P) OPT=(TS, NOEIGEN, CALCFC) \\semicis Csglyoxylb3lyp/6 $-31 \mathrm{G}(\mathrm{d}, \mathrm{p}) \backslash \backslash 0,2 \backslash 0,0 .,-1.2854986521,1.2172365353 \backslash \mathrm{C}, 0$. , $-0.247852701,0.385$ $0843891 \backslash 0,0$. $0.9750827515,0.7947634018 \backslash \mathrm{C}, 0 .,-0.3368686823,-1.032335702$ $7 \backslash 0,0.0 .8516919852,-1.5477807078 \backslash \mathrm{H}, 0 .,-1.2464250636,-1.6235685666 \backslash \mathrm{H}, 0$ ., $-0.9408345321,2.1243892358 \backslash \mathrm{H}, 0 ., 1.3653792181,-0.3310666226 \backslash \backslash$ Version=

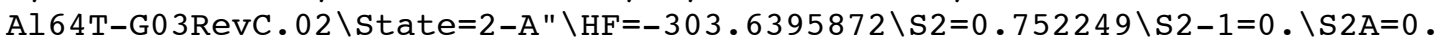
$750004 \backslash \mathrm{RMSD}=4.512 \mathrm{e}-09 \backslash \mathrm{RMSF}=8.570 \mathrm{e}-05 \backslash \mathrm{Dipole}=0 .,-0.6821002,0.7852533 \backslash \mathrm{PG}$ $=\mathrm{CS}[\mathrm{SG}(\mathrm{C} 2 \mathrm{H} 3 \mathrm{O} 3)] \backslash \backslash @$

\section{0}

$1 \backslash 1 \backslash G I N C-L C 71 \backslash F O p t \backslash U B 3 L Y P \backslash 6-31 G(d, p) \backslash C 2 H 303(2) \backslash G M S 501 \backslash 05-J a n-2005 \backslash 0 \backslash \backslash \#$ B3LYP/6-31G(D,P) OPT=TIGHT FREQ=NORAMAN $\backslash \backslash \mathrm{S} 1$ hydrated cis C2v glyoxyl

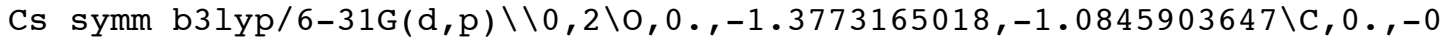
$.1818959594,-0.4473191512 \backslash 0,0 ., 0.9048041714,-1.0318072306 \backslash \mathrm{C}, 0 .,-0.2604$ $121959,0.9840489724 \backslash 0,0$. $0.897966939,1.6516701531 \backslash \mathrm{H}, 0 .,-1.1655866598,1$


$10761 \backslash \backslash$ Version=IA32L-G03RevC.02 \State $=2-A " \backslash H F=-303.6615969 \backslash S 2=0.754368$ $\backslash S 2-1=0 . \backslash S 2 A=0.750013 \backslash R M S D=3.291 e-09 \backslash R M S F=2.626 e-06 \backslash D i p o l e=0 .,-0.38774$ $36,-0.0961631 \backslash P G=C S \quad[S G(C 2 H 303)] \backslash \backslash @$

\section{TS:20 $\rightarrow 21$}

$1 \backslash 1 \backslash G I N C-S C 125 \backslash F T S \backslash U B 3 L Y P \backslash 6-31 G(d, p) \backslash C 4 H 9 O 4(2) \backslash G M S 501 \backslash 06-J a n-2005 \backslash 0 \backslash \backslash \#$ B3LYP/6-31G (D,P) OPT=(TS, NOEIGEN, CALCFC) FREQ=NORAMAN $\backslash \backslash T S$ Ado=ethanol


$, 0.461892614 \backslash \mathrm{C},-1.1657680602,0.6461853008,0.4017520944 \backslash \mathrm{H}, 0.0699609976$, $0.4938687804,0.5416216585 \backslash \mathrm{H},-1.5818724608,0.0864741828,1.2436318677 \backslash \mathrm{C}$, $-1.4767495466,0.0406034043,-0.9262083707 \backslash 0,-1.7518961422,0.7050829248$, $-1.9090197769 \backslash 0,-1.3408875883,-1.302604972,-0.9453844965 \backslash \mathrm{H},-1.50319834$ $5,-1.5840591739,-1.8617032881 \backslash \mathrm{H},-1.5872176121,2.2824033934,-0.46171811$ $31 \backslash \mathrm{C}, 1.8898368668,-1.0477978701,0.8935441118 \backslash \mathrm{C}, 1.5682552027,0.41310947$ $03,0.7650747044 \backslash \mathrm{H}, 1.5446571156,-1.5866010832,-0.0026185852 \backslash \mathrm{H}, 1.3547871$ $887,-1.4745016157,1.7568124138 \backslash 0,3.306748501,-1.1804766945,1.056205516$ $8 \backslash \mathrm{H}, 1.674025983,1.0142404119,1.6672539303 \backslash \mathrm{H}, 1.9389807482,0.9048070103$, $-0.1341550393 \backslash \mathrm{H}, 3.5044975382,-2.1236215855,1.1363490568 \backslash \backslash$ Version=Al64 T -G03RevC.02 \State $=2-\mathrm{A} \backslash \mathrm{HF}=-458.6606091 \backslash \mathrm{S} 2=0.756674 \backslash \mathrm{S} 2-1=0 . \backslash \mathrm{S} 2 \mathrm{~A}=0.750026$ $\backslash \mathrm{RMSD}=8.907 e-09 \backslash \mathrm{RMSF}=6.958 e-06 \backslash \mathrm{Dipole}=-0.1000258,-1.1421583,-0.1526367$ $\backslash \mathrm{PG}=\mathrm{C} 01[\mathrm{X}(\mathrm{C} 4 \mathrm{H} 904)] \backslash \backslash @$ 


\section{1}

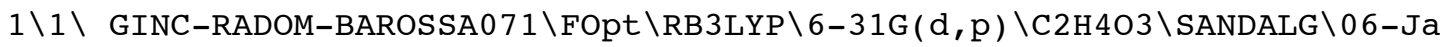
n-2005 \0\\\# B3LYP/6-31G(D,P) OPT=TIGHT FREQ=NORAMAN \\Cssymm hydroxyac

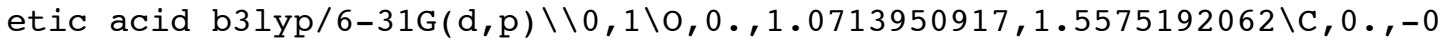
$.2278099345,1.0320239066 \backslash \mathrm{H}, 0.8826152754,-0.796458312,1.3618355798 \backslash \mathrm{H},-0$ $.8826152754,-0.796458312,1.3618355798 \backslash \mathrm{C}, 0 .,-0.1995011005,-0.4834692099$ $\backslash 0,0 ., 0.82988273,-1.126585388 \backslash 0,0 .,-1.4271276101,-1.0321267664 \backslash \mathrm{H}, 0 .,-1$ $.30583096,-1.9969972928 \backslash \mathrm{H}, 0 ., 1.6694121012,0.791541538 \backslash \backslash$ Version=x86-Lin ux-G03RevB.02 $\backslash$ State $=1-A^{\prime} \backslash \mathrm{HF}=-304.299873 \backslash \mathrm{RMSD}=4.232 \mathrm{e}-09 \backslash \mathrm{RMSF}=4.584 \mathrm{e}-06 \backslash$ Dipole $=0 .,-0.7367134,-0.5751871 \backslash P G=C S \quad[S G(\mathrm{C} 2 \mathrm{H} 2 \mathrm{O} 3), \mathrm{x}(\mathrm{H} 2)] \backslash \backslash @$ 
TABLE S2: G3(MP2)-RAD//B3-LYP/6-31G(d,p)

Total Energies (Hartrees) at $0 \mathrm{~K}$

\begin{tabular}{|c|c|}
\hline Species & Energy \\
\hline $1 \mathbf{a}$ & -229.93235 \\
\hline TS:1a $\rightarrow \mathbf{3 a}$ & -384.04079 \\
\hline $3 \mathbf{a}$ & -229.28143 \\
\hline $4 \mathbf{a}$ & -229.28735 \\
\hline TS: $4 a \rightarrow 5 a$ & -384.05490 \\
\hline $5 \mathbf{a}$ & -229.95060 \\
\hline $2 a$ & -153.59938 \\
\hline $1 \mathrm{~b}$ & -269.17343 \\
\hline TS: $1 b \rightarrow 3 b$ & -423.28253 \\
\hline $3 \mathbf{b}$ & -268.52010 \\
\hline $4 b$ & -268.52576 \\
\hline TS: $4 b \rightarrow 5 b$ & -423.29267 \\
\hline $5 b$ & -269.18423 \\
\hline $2 \mathbf{b}$ & -192.83336 \\
\hline $6 \mathbf{a}$ & -152.94770 \\
\hline TS: $6 a \rightarrow 2 a$ & -307.70741 \\
\hline $6 \mathrm{~b}$ & -192.18948 \\
\hline TS: $6 b \rightarrow 2 b$ & -346.94596 \\
\hline $\mathrm{H}_{2} \mathrm{O}$ & -76.34253 \\
\hline Ethanol & -154.79020 \\
\hline Ethanol radical & -154.12882 \\
\hline 1a with $K^{+}$ & -829.23286 \\
\hline TS:1a with $\mathrm{K}^{+} \rightarrow 2 \mathrm{a}$ with $\mathrm{K}^{+}$ & -983.34058 \\
\hline
\end{tabular}


$2 \mathrm{a}$ with $\mathrm{K}^{+}$

3a with $\mathrm{K}^{+}$

TS:3a with $\mathrm{K}^{+} \rightarrow 4 \mathrm{a}$ with $\mathrm{K}^{+}$

$4 a$ with $K^{+}$

FH॰••1a

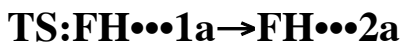

FH•••2a

FH॰••3a

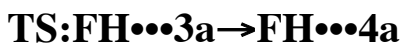

FH॰••4a

$\mathrm{NH}_{4}^{+} \bullet \bullet \bullet \mathbf{a}$

$\mathrm{TS}: \mathrm{NH}_{4}^{+} \bullet \bullet \bullet 1 \mathrm{a} \rightarrow \mathrm{NH}_{4}^{+} \bullet \bullet \bullet 2 \mathrm{a}$

$\mathbf{N H}_{4}^{+} \bullet \bullet \bullet 2 a$

$\mathrm{NH}_{4}^{+} \bullet \bullet \cdot 3 a$

$\mathrm{TS}: \mathrm{NH}_{4}^{+} \bullet \bullet \bullet 3 \mathrm{a} \rightarrow \mathrm{NH}_{4}^{+} \bullet \bullet \bullet 4 a$

$\mathrm{NH}_{4}^{+} \bullet \bullet 4 a$

$1 \mathrm{a} \bullet \bullet \bullet \mathrm{NH}_{3}$

TS: $1 \mathrm{a}^{\bullet \bullet \bullet} \mathrm{NH}_{3} \rightarrow \mathbf{2 a} \bullet \bullet \bullet \mathrm{NH}_{3}$

$2 \mathrm{a} \bullet \bullet \bullet \mathrm{NH}_{3}$

$3 \mathrm{a} \bullet \bullet \bullet \mathrm{NH}_{3}$

$\mathrm{TS}: 3 \mathrm{a}^{\bullet \bullet \bullet} \mathrm{NH}_{3} \rightarrow 4 \mathrm{a}^{\bullet \bullet \bullet \mathrm{NH}_{3}}$

$4 \mathbf{a} \bullet \bullet \mathrm{NH}_{3}$

$1 \mathrm{a} \bullet \bullet \mathrm{OCHO}^{-}$

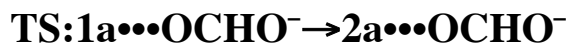

$2 \mathrm{a} \cdot \bullet \cdot \mathrm{OCHO}^{-}$

$3 \mathrm{a} \bullet \bullet \mathrm{OCHO}^{-}$
$-828.57727$

$-828.58049$

$-983.34992$

$-829.24403$

$-330.30213$

$-484.41043$

$-329.64939$

$-329.65510$

$-484.42262$

$-330.31895$

$-286.76393$

$-440.87198$

$-286.11122$

$-286.10991$

$-440.87945$

$-286.77467$

$-286.41107$

$-440.52169$

$-285.76138$

$-285.76659$

$-440.53461$

$-286.42961$

$-418.95820$

$-497.93520$

$-418.31359$

$-418.32350$ 


\begin{tabular}{|c|c|}
\hline 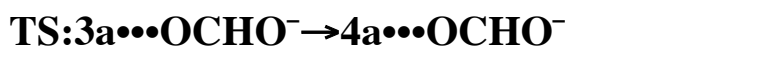 & -497.95243 \\
\hline $4 \mathbf{4} \bullet \bullet \mathrm{OCHO}^{-}$ & -418.98658 \\
\hline 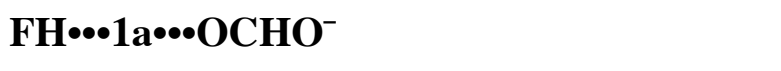 & -519.33906 \\
\hline 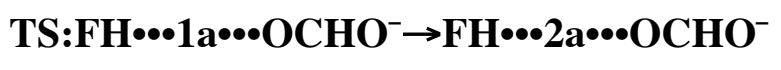 & -598.31513 \\
\hline $\mathbf{F H} \bullet \bullet \cdot 2 \mathrm{a} \bullet \bullet \bullet \mathrm{OCHO}^{-}$ & -518.69456 \\
\hline 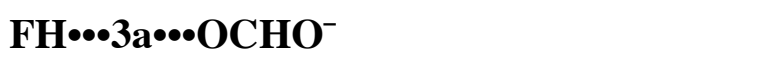 & -518.69206 \\
\hline 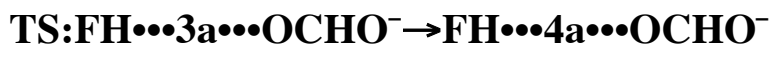 & -598.32327 \\
\hline $\mathbf{F H} \bullet \bullet 4 a \bullet \bullet \bullet \mathrm{OCHO}^{-}$ & -519.35635 \\
\hline $\mathrm{NH}_{4}^{+} \bullet \bullet \bullet 1 \mathrm{a} \bullet \bullet \bullet \mathrm{NH}_{3}$ & -343.25172 \\
\hline $\mathrm{TS}: \mathrm{NH}_{4}^{+} \bullet \bullet \bullet 1 \mathrm{a} \bullet \bullet \bullet \mathrm{NH}_{3} \rightarrow \mathrm{NH}_{4}^{+} \bullet \bullet \bullet 2 \mathrm{a}^{\bullet \bullet \bullet} \mathrm{NH}_{3}$ & -497.36072 \\
\hline $\mathrm{NH}_{4}^{+} \bullet \bullet \bullet 2 \mathrm{a} \bullet \bullet \bullet \mathrm{NH}_{3}$ & -342.60248 \\
\hline $\mathrm{NH}_{4}^{+} \bullet \bullet \bullet 3 \mathrm{a} \bullet \bullet \bullet \mathrm{NH}_{3}$ & -342.60036 \\
\hline $\mathrm{TS}: \mathrm{NH}_{4}^{+} \bullet \bullet \cdot 3 \mathrm{a} \bullet \bullet \bullet \mathrm{NH}_{3} \rightarrow \mathrm{NH}_{4}^{+} \bullet \bullet \bullet 4 a \bullet \bullet \bullet \mathrm{NH}_{3}$ & -497.36939 \\
\hline $\mathrm{NH}_{4}^{+} \bullet \bullet \bullet 4 a \bullet \bullet \bullet \mathrm{NH}_{3}$ & -343.26444 \\
\hline $7 \mathbf{a}$ & -305.09337 \\
\hline $\mathrm{TS}: 7 \mathbf{a} \rightarrow \mathbf{8 a}$ & -459.20131 \\
\hline $\mathbf{8 a}$ & -304.44042 \\
\hline $9 \mathbf{a}$ & -228.11174 \\
\hline $10 \mathbf{a}$ & -228.09777 \\
\hline TS:9a $\rightarrow 11 a$ & -382.85504 \\
\hline $11 a$ & -228.73977 \\
\hline $\mathrm{CN}^{-} \bullet \bullet \bullet 9 \mathrm{a}$ & -320.90352 \\
\hline TS:CN- $\mathbf{N}^{-} \bullet \bullet 9 a$ & -320.90354 \\
\hline $\mathrm{HCOO}^{-} \bullet \bullet 9 \mathrm{a}$ & -417.14298 \\
\hline TS:HCOO-•••9a & -417.13765 \\
\hline $\mathrm{CHONH}_{2} \bullet \bullet 9 a$ & -397.78709 \\
\hline
\end{tabular}




\begin{tabular}{|c|c|}
\hline TS:CHONH${ }_{2} \bullet \bullet 9 a$ & -397.76771 \\
\hline Imidazole•••9a & -453.99853 \\
\hline TS:Imidazole•••9a & -453.97774 \\
\hline $7 \mathbf{b}$ & -344.33763 \\
\hline $7 b^{*}$ & -344.33420 \\
\hline $8 b$ & -343.68280 \\
\hline $\mathbf{8 b} *$ & -343.68205 \\
\hline $9 b$ & -267.35722 \\
\hline $9 b^{*}$ & -267.35638 \\
\hline $10 b$ & -267.34432 \\
\hline $11 b$ & -267.98634 \\
\hline $11 b^{*}$ & -267.97969 \\
\hline 12 & -612.75363 \\
\hline 13 & -689.10912 \\
\hline TS: $13 \rightarrow 14$ & -843.21808 \\
\hline 14 & -688.44970 \\
\hline 15 & -688.44756 \\
\hline HCl & -460.35291 \\
\hline 16 & -227.53660 \\
\hline 17 & -303.89266 \\
\hline $\mathrm{TS}: 17 \rightarrow 18$ & -458.00797 \\
\hline 18 & -303.27029 \\
\hline 19 & -303.26297 \\
\hline 20 & -303.28623 \\
\hline $\mathrm{TS}: 20 \rightarrow 21$ & -458.03543 \\
\hline 21 & -303.92173 \\
\hline
\end{tabular}




\section{COMPLETE CITATIONS}

(33) MOLPRO 2002.6 is a package of ab initio programs written by Werner, H.-J.;

Knowles, P. J.; Schütz, M.; Lindh, R.; Celani, P.; Korona, T.; Rauhut, G.; Manby, F. R.; Amos, R. D.; Bernhardsson, A.; Berning, A. Cooper, D. L.; Deegan, M. J. O.; Dobbyn, A. J.; Eckert, F.; Hampel, C.; Hetzer, G.; Lloyd, A. W.; McNicholas, S. J.; Meyer, W.; Mura, M. E.; Nicklaß, A.; Palmieri, P; Pitzer, R.; Schumann, U.; Stoll, H.; Stone, A. J.; Tarroni, R.; Thorsteinsson, T.

(34) Frisch, M. J.; Trucks, G. W.; Schlegel, H. B.; Scuseria, G. E.; Robb, M. A.; Cheeseman, J. R.; Montgomery, J. A., Jr.; Vreven, T.; Kudin, K. N.; Burant, J. C.; Millam, J. M.; Iyengar, S. S.; Tomasi, J.; Barone, V.; Mennucci, B.; Cossi, M.; Scalmani, G.; Rega, N.; Petersson, G. A.; Nakatsuji, H.; Hada, M.; Ehara, M.; Toyota, K.; Fukuda, R.; Hasegawa, J.; Ishida, M.; Nakajima, T.; Honda, Y.; Kitao, O.; Nakai, H.; Klene, M.; Li, X.; Knox, J. E.; Hratchian, H. P.; Cross, J. B.; Adamo, C.; Jaramillo, J.; Gomperts, R.; Stratmann, R. E.; Yazyev, O.; Austin, A. J.; Cammi, R.; Pomelli, C.; Ochterski, J. W.; Ayala, P. Y.; Morokuma, K.; Voth, G. A.; Salvador, P.; Dannenberg, J. J.; Zakrzewski, V. G.; Dapprich, S.; Daniels, A. D.; Strain, M. C.; Farkas, O.; Malick, D. K.; Rabuck, A. D.; Raghavachari, K.; Foresman, J. B.; Ortiz, J. V.; Cui, Q.; Baboul, A. G.; Clifford, S.; Cioslowski, J.; Stefanov, B. B.; Liu, G.; Liashenko, A.; Piskorz, P.; Komaromi, I.; Martin, R. L.; Fox, D. J.; Keith, T.; Al-Laham, M. A.; Peng, C. Y.; Nanayakkara, A.; Challacombe, M.; Gill, P. M. W.; Johnson, B.; Chen, W.; Wong, M. W.; Gonzalez, C.; Pople, J. A., Gaussian, Inc., Pittsburgh, PA, 2003. 